\title{
Final Report for the Iowa Livestock Industry Waste Characterization and Methane Recovery Information Dissemination Project

$$
\text { DE-FGO2-99EE } 35129
$$

By

M. V. Garrison

T. L. Richard

Contracted by

lowa Department of Natural Resources

Energy \& Geological Resources Division

Wallace State Office Building

Des Moines, IA 50319-0034

\author{
Project Manager \\ Thomas L. Richard \\ Department of Agricultural \& Biosystems Engineering \\ Iowa State University \\ 102 Davidson Hall \\ Ames, IA 50011 \\ Co-Investigators: \\ Wendy Powers-Schilling \\ Department of Animal Science \\ Michael Burkart \\ National Soil Tilth Laboratory \\ Contract No. \\ 00-6147-01
}

This Final Report was prepared with a grant from the U. S. Department of Energy (DOE) for the State Energy Program; Petroleum Violation Escrow Funds. However, any opinions, findings, conclusions, or recommendations expressed herein are those of the authors and do not necessarily reflect the view of DOE. 


\section{DISCLAMMER}

This report was prepared as an sccount of work sponsored by an agency of the United States Government. Neitber the United States Governmeat nor any agency thereof, nor any of their employees, makes any warranty, express or implied, of essumes any legal liability or responsibility for the accuracy, completeness, or usefulness of any information, apparatus, product, or process disclosed, or represents that its use would not infringe privately owned rights. Refereace herein to any specific commercial product, process, or service by trade name, trademart, manufacturer, or otherwise does not necessarily constitute or imply its eadorsement, recommendation, of favoriag by the Uaited States Government or any agency thereof. The riews and opinions of authors expressed herein do not necessarily state or reflect those of the United States Government or any agency thereof. 


\section{DISCLAIMER}

Portions of this document may be illegible in electronic image products. Images are produced from the best available original document. 


\section{Table of Contents}

Introduction 1

Objective 1

Methodology 1

Waste Characterization $\quad 2$

Beef $\quad 3$

Dairy $\quad 3$

Swine : 4

Poultry 5

Economic Feasibility of Methane Recovery 5

$\begin{array}{ll}\text { Fossil Fuel Displacement } & 7\end{array}$

$\begin{array}{ll}\text { Market Assessment } & 7\end{array}$

$\begin{array}{ll}\text { Conclusions } & 8\end{array}$

References $\quad 9$

Appendix A - Tables 11

$\begin{array}{ll}\text { Appendix B - Figures } & 24\end{array}$

Appendix C - State \& County Waste Inventory 


\section{List of Tables}

Table 1 Summary of Potential Livestock Methane Energy in Iowa (based on county animal census data)

Table 2 Iowa Department of Natural Resources Permitted Facility Waste Management Profile

Table 3 EPA Waste Handling and Management for Livestock Types in Iowa

Table 4 Swine Herd Percentages and Waste Management Strategies

Table 5 Feedlot Manure Disposal Based on Percentage of Operation and Cows

Table 6 Cow-Calf Operations Manure Disposal Methods by \% of Operations

Table 7 Cow-Calf Operations Manure Disposal Methods by \% of Manure

Table 8 Dairy Housing Systems Based on Animal Types

Table 9 Dairy Milking Facility Type Based on \% of Operations and Cows

Table 10 Type of Dairy Manure Collection System

Table 11 Type of Dairy Manure Management or Storage System

Table $12 \%$ of Dairy Lagoon Systems with Single or Multiple Stages

Table $13 \%$ of Dairy Lagoons or Tank Storages with Solid Separation

Table 14 Dairy Manure Disposal Method

Table 15 Frequency of Dairy Manure Application

Table 16 Dairy Manure Application Method

Table 17 Dairy Manure Application Method

Table 18 Production Phases Within Swine Operations

Table 19 Facility Types in Each Production Phase by \% of Operations and Hogs

Table 20 Type of Grower/Finisher Facility Waste Handling System 
Table 21 Grower/Finisher Operation Flooring Types 16

Table 22 Grower/Finisher Waste Storage Systems 16

Table 23 Grower/Finisher Operations Disposing of Liquid/Solid Waste or 16 Unseparated Waste

Table 24 Manure Handling Methods for Layer Operations in the Central U.S. 17

$\begin{array}{lll}\text { Table } 25 & \text { AgSTAR Model Scenario Descriptions } & 17\end{array}$

Table 26 Other AgSTAR Model Input Values $\quad 17$

Table 27 Swine Farrow to Finish System Energy Production and Costs 17

Table 28 Swine Farrow to Finish System Methane Recovery Economics 18

Table 29 Swine Farrow to Finish System Methane Recovery Economics 18

Table 30 Swine Finishing System Energy Production and Costs 18

$\begin{array}{lll}\text { Table } 31 \text { Swine Finishing System Methane Recovery Economics } & 19\end{array}$

Table 32 Swine Finishing System Methane Recovery Economics . 19

Table 33 Dairy Tie Stall with Plug Flow Digester Energy Production and 19 Costs

Table 34 Dairy Tie Stall with Plug Flow Digester Methane Recovery 20 Economics

Table 35 Dairy Tie Stall with Plug Flow Digester Methane Recovery 20 Economics

Table 36 Dairy Tie Stall with Complete Mix Digester Energy Production and 20 Costs

Table 37 Dairy Tie Stall with Complete Mix Digester Methane Recovery 20 Economics

Table 38 Dairy Tie Stall with Complete Mix Digester Methane Recovery 21 Economics

Table 39 Dairy Free Stall with Plug Flow Digester Energy Production and 21 Costs 
Table 40 Dairy Free Stall with Plug Flow Digester Methane Recovery

Economics

Table 41 Dairy Free Stall with Plug Flow Digester Methane Recovery

Economics

Table 42 Dairy Free Stall Complete Mix Digester Energy Production and

22 Costs

Table 43 Dairy Free Stall Complete Mix Digester Methane Recovery Economics

Table 44 Dairy Free Stall Complete Mix Digester Methane Recovery Economics

Table 45 Economic Breakeven Points for Methane Recovery Facilities

Table 46 Importance Rankings of Methane Meeting Participant and Producer Opinions of Livestock Methane Recovery

Table 47 Policy Scenarios for the Fossil Fuel Displacement

Table 48 Quantities of Fossil Fuels Displaced by Livestock Methane Recovery 


\section{List of Figures}

Figure 1 Farm and Inventory Based on USDA Beef Herd Classifications 25

Figure 2 Farm and Inventory Based on USDA Dairy Herd Classifications 25

Figure 3 Swine Herd Profiles for Iowa 26

Figure 4 Profile for Poultry Layer Farm Sizes 26

Figure 5 Net Present Value of Swine Farrow to Finish Economics $\quad 27$

Figure 6 Net Present Value of Swine Farrow to Finish Operations 28

Figure 7 Net Present Value of Finishing Operations 29

Figure 8 Net Present Value of Finishing Operations 30

Figure 9 Farrow to Finish System Manure Production 31

Figure 10 Farrow to Finish Operation Component and Total Costs 32

Figure 11 Finishing Operation Component and Total Costs . 33

Figure 12 Farrow to Finish and Finishing System Gas Production 34

Figure 13 Swine Farrow to Finish and Finishing Maximum Energy Production 35

Figure 14 Net Present Value of Dairy Tie Stall with Plug Flow Digester 36

Figure 15 Net Present Value of Dairy Tie Stall with Plug Flow Digester $\quad 37$

Figure 16 Net Present Value of Dairy Tie Stall with Complete Mix Digester $\quad 38$

Figure 17 Net Present Value of Dairy Tie Stall with Complete Mix Digester $\quad 39$

Figure 18 Net Present Value of Dairy Free Stall with Plug Flow Digester $\quad 40$

Figure 19 Net Present Value of Dairy Free Stall with Plug Flow Digester $\quad 41$

Figure 20 Net Present Value of Dairy Free Stall with Complete Mix Digester $\quad 42$

Figure 21 Net Present Value of Dairy Free Stall with Complete Mix Digester 43

Figure 22 Dairy Tie and Free Stall Manure Production 44 
Figure 23 Dairy Tie Stall Plug Flow Digester Component and Total Costs

Figure 24 Dairy Tie Stall Complete Mix Digester Component and Total Costs 46

Figure 25 Dairy Free Stall Plug Flow Digester Component and Total Costs 47

Figure 26 Dairy Free Stall Complete Mix Digester Component and Total Costs 48

Figure 27 Dairy System Gas Production 49

Figure 28 Dairy System Maximum Energy Production 50

Figure 29 Electricity output (MW) per county if all available dairy manure generated methane is combusted in an engine generator $(30 \%$ efficiency) to generate electricity.

Figure 30 Electricity output (MW) per county if all available swine manure generated methane is combusted in an engine generator $(30 \%$ efficiency) to generate electricity.

Figure 31 Maximum Number of Farms that Meet Dairy Herd Size Requirements in Table 45 for Scenario \#3 (Top) and Scenario \#6 (Bottom)

Figure 32 Maximum Number of Farms that Meet Swine Herd Size Requirements in Table 45 for Scenario \#3 (Top) and Scenario \#6 (Bottom) 


\section{Introduction}

The Iowa livestock industry generates large quantities of manure and other organic residues; composed of feces, urine, bedding material, waste feed, dilution water, and mortalities. Often viewed as a waste material, little has been done to characterize and determine the usefulness of this resource. The Iowa Department of Natural Resources initiated the process to assess in detail the manure resource and the potential utilization of this resource through anaerobic digestion coupled with energy recovery.

Many of the pieces required to assess the manure resource already exist, albeit in disparate forms and locations. This study began by interpreting and integrating existing Federal, State, ISU studies, and other sources of livestock numbers, housing, and management information. With these data, models were analyzed to determine energy production and economic feasibility of energy recovery using anaerobic digestion facilities on livestock farms. Having these data individual facilities and clusters that appear economically feasible can be identified specifically through the use of a GIS system for further investigation. Also livestock facilities and clusters of facilities with high methane recovery potential can be the focus of targeted educational programs through Cooperative Extension network and other outreach networks, providing a more intensive counterpoint to broadly based educational efforts.

\section{Objective}

The purpose of this document is to summarize analytical methods, characterize Iowa livestock wastes, determine fossil fuel displacement by methane use, assess the market potential, and offer recommendations for the implementation of methane recovery technologies.

\section{Methodology}

The following outlines the methods for the Iowa Livestock Industry Waste Characterization/Assessment Project. Generally, the methods were designed to collect farm scale census and survey data and to incorporate it into models of methane recovery potential to determine the economic feasibility of methane recovery from Iowa livestock facilities.

- Collect survey and census data on facility size, livestock numbers, and manure management types.

- Classify facilities by general animal type (i.e. swine, dairy, etc), herd size, and housing/confinement manure management categories.

- Connect the housing/confinement type to the most appropriate methane generation scheme (i.e. covered lagoons, complete mix digesters, plug flow digesters, sequencing batch reactors, etc).

- Use methane recovery models, developed by the AgSTAR program of the U.S. EPA (EPA, 1997), to determine the economic feasibility of each facility category for methane recovery. 
- Conduct a detailed analysis examining the effects of the price of heating oil and electricity and the resultant benefits for the increased utilization of methane to displace these energy sources.

- Calculate the potential fossil fuel displacement using various economic and resource availability scenarios.

- Conduct a "market assessment" that identifies the roles, strategies, and future steps for stakeholders to encourage the implementation of anaerobic digestion and methane recovery.

- Publish a final report, which details the project methodologies, results, and conclusions.

\section{Waste Characterization}

A literature search was conducted to locate and evaluate various data sources related to livestock inventories and the distribution of manure management systems. U.S. Agricultural Statistic data, an ISU Economics Survey, and IDNR permit information were used to create a distribution of facility types (size, waste management, etc). On a state level the size distribution of livestock operations was calculated along with the treatment regimes that were available from EPA sources. For the large operations the IDNR construction permit sets were used to profile the proportions of the manure management systems. State manure management plans were not included in this analysis since they contain very little pertinent information.

The table appendix contains the tables summarized from various Animal and Plant Health Inspection Service (APHIS) and EPA reports that characterize waste management in the U.S., including lowa, for various animal types. Since the tables were drawn from many different sources, there are some differences on the actual percentages of the individual waste systems. Some of the differences can be attributed to differences in the definition of the waste systems or whether the percentage is expressed on a percent operation or percent animal basis. Appendix A contains the data that utilized U.S. and State Agricultural Census information for determining state and county level total potential energy production from methane and waste generation from each animal species. Calculations of energy potential followed the procedures contained in a WRBEP report (NEOS, 1994). Table 1 , in the table appendix, summarizes the potential livestock methane energy in Iowa.

For each county the total number of livestock facilities was divided by type (swine, dairy, etc.). For each livestock type we have developed a distribution of facility sizes and manure systems for the county using a combination of U.S. Ag. Statistics data, the ISU Economics survey (for swine), and IDNR permit data. These charts, which show distribution of livestock numbers, are incorporated with the APHIS information in the tables in the Appendix A. IDNR permit data was used to estimate proportions of manure management systems for the larger facility categories, which were not well represented in the Economics survey and should have been represented in the permit listing if they were built or expanded in the last 15 years. The Iowa Department of Natural Resource permit database was mined to determine common treatment regimes based on animal type. 
Tables 2 \& 3 show a simplified breakdown of treatment regime based on IDNR and U.S. Environmental Protection categories for livestock waste management systems. Table 4 further details the waste management and herd percentages for the swine category as derived from the ISU Economics survey.

\section{Beef}

Iowa beef industry, according to 1997 statistics, was comprised of approximately $1,029,172$ cows down from 1,065,744 cows during 1992 (USDA, 1997). These figures do not include heifers and heifer calves. Figure 1 shows the farm and herd profiles for Iowa beef herds. Most of the operations have less than 50 cows while the majority of cows are in operations having 50 or more cows.

Tables 5 through 7 detail the methods used by beef operations to dispose of their manure. This information was obtained from APHIS reports for beef cow-calf and feedlot management. The cow-calf study involved 23 states, including Iowa, which had 85.0 percent of all of the beef cows in the U.S. and 66.3 percent of the beef cow operations. The feedlot study involved 12 western states, including Iowa, which contain 82.1 percent of all cattle on feed in operations of 1,000 head or more. As the APHIS results were not broken out by individual states, we applied the multi-state values to the Iowa herd size distribution. The tables note most of the manure generated in cow-calf operations was applied to land owned or rented by the operation.

\section{Dairy}

Iowa dairy industry, according to 1997 statistics, was comprised of approximately 222,142 cows down from 258,925 cows during 1992 (USDA, 1997). These figures only consider milking cows and not dry cows, bulls, heifers, etc. Figure 2 shows the farm and herd profiles for the Iowa dairy herds. Vast majority of farms had 100 or fewer lactating cows in the operation and almost $45 \%$ of the cow inventories were in herds of 50 to 99 head.

Dairy management practices, including waste management, for Iowa and 19 other states were profiled in a 1996 three-part APHIS study. These states comprise $83.1 \%$ of the U.S. milk cow inventory and lowa, when considered alone, has $2.6 \%$ of the U.S. milk cow inventory (APHIS, 1996). As with the beef cattle APHIS data, the management practices are not broken out specifically for Iowa, so the multi-state distribution are presented. Tables 8 and 9 list the housing and milking facility types for operations. For unweaned dairy heifers most of the animals are being housed in individual or multiple animal areas, while the majority of the weaned dairy heifers are housed on pasture, dry lot, or multiple animal areas. Lactating cows in most operations have access to tie stall or stanchion areas, dry lot, or pasture. For milking the vast majority of operations use either a tie stall or stanchion system, but based on the percentage of cows, the majority of operations use a parlor system. This indicates at the larger size farms parlors are more popular than a tie stalls or stanchions.

Tables 10 through 13 from the same APHS reports provide insight into the types of manure collection, management, and/or storage methods for the dairy industry. Most 
of the operations less than 200 head used either a gutter or alley scraper while the larger systems tended to use water flushed alley. Manure from scraped systems will have higher solids content compared to a water flushed system. The scraped systems, with higher solids, would be compatible with plug flow digesters, while the water-flushed systems would need either solids separation for plug flow digesters or could use complete mix digesters without solids separation. Solid manure handling systems seem to be fairly common no matter the size of the cowherd (table 11), although slurry and anaerobic lagoon systems became more prominent in the larger cowherds. From table 12 it is clear that most operations with a lagoon only have a single-stage lagoon for holding or treating the dairy waste. Of the systems with lagoon or tank storage, only $29.5 \%$ have any type of solid/liquid separation (table 13).

Dairy manure application details are contained in tables 14 through 17 from the APHIS dairy reports. These tables note the means of disposal (table 14), frequency of application (table 15), method of application (table 16), and specific management practices (table 17). The vast majority of the operations applied the dairy waste directly to land owned or rented by the operation. Frequency of application during the summer months was broadly distributed between daily, weekly, monthly or less, but during the winter months application bimodally distributed with $46 \%$ of operations daily and $31 \%$ of operations spreading less than once a month. This bimodal distribution presumably reflects the availability of adequate storage and in some of the states, such as California, can apply year around on a daily basis, as indicated in Table 11. In Iowa the less than monthly basis is probably more common than in other states in this study due to the weather conditions experienced here. Most common application method, across all herd sizes, was a broadcast/solid spreader method. Over 100 head, due to the greater prevalence of liquid storage systems, the slurry/surface application also was common. Concerning specific manure management practices, many producers established application rates based on manure nutrient content or crop needs and a majority of operators applied at least 50 feet from a water body.

\section{Swine}

Iowa swine industry, according to 1997 statistics, was comprised of approximately 14,651,919 pigs up from 14,153,158 pigs during 1992 (USDA, 1997). Figure 3 below shows the farm and herd profiles for the Iowa swineherds. A majority of the farms have 200 or more hogs, but the majority of hogs are on farms having 1,000 head or more.

Tables 18 through 23 outline the characteristics of the swine industry from two APHIS reports, Part I: Reference of 1995 Swine Management Practices and Part II: Reference of 1995 U.S. Grower/Finisher Health \& Management Practices. Tables 18 and 19 typify the production phase and the facility type typically used for each swine production phase. Most operations have farrowing and/or grower/finisher operations but only slightly less than half have nursery facilities. Tables 20 through 23 offer some insight into the types of swine manure storage and handling strategies used by producers. Tables 21,22 , and 23 note the type of flooring, waste storage system, and solid/liquid separation details for grower/finisher operations. For grower/finisher majority of 
operations are using either deep pit, above or below ground storage, or anaerobic lagoon systems. With grower/finisher systems larger than 10,000 head, anaerobic lagoons without covers tended to dominate.

Poultry

Iowa poultry in 1997 consisted of 24,876,834 layers, 1,017,224 broilers, and 2,552,624 turkeys. The total number of farms was 2,655, down from 3,390 in 1992. Broiler and turkey numbers decreased from 1992 to 1997 but the number of layers nearly doubled. Figure 4 shows the size profile of layer operations in Iowa as taken from the agricultural statistics data (USDA, 1997). Table 24 lists the types of manure handling systems from an APHIS study of layer operations in the central region of the U. S., including Iowa. As one can see from the table most of the layer operations in this region are high-rise, manure belt, or scraper systems. These types of systems would not be very compatible with a methane recovery system. Difficulties include the low moisture content of the manure and the difficulty of capturing the methane due to the housing or manure storage system design.

\section{Economic Feasibility of Methane Recovery}

Economic models developed by the US EPA AgSTAR program were used to identify facilities where anaerobic digestion and methane recovery technology might be cost effective. For swine farrow to finish, finishing, dairy tie stall, and dairy free stall operation models were run to calculate methane production, possible generator production, system costs, net present value (NPV), and payback periods for methane recovery facilities under six energy and economic scenarios.

Six scenarios were developed for input to the AgSTAR model representing variations in electricity rates and economic incentives. AgSTAR then calculated the cost and profitability of anaerobic digestion facilities, with results in Table 25 (US EPA 1997). Economic scenarios 1 to 3 had electricity rates of $\$ 0.06, \$ 0.08$, and $\$ 0.12$ per $\mathrm{kWh}$, respectively, with no heating cost contribution, a loan rate of $10 \%$, and producer downpayment of $20 \%$ of the system cost. Scenario 4 considered electricity rates of $\$ 0.06$ per $\mathrm{kWh}$ with $90 \%$ of the $\$ 1.00$ per gallon liquid propane on-farm heating needs displaced by generator heat recovery. Scenarios 5 and 6 also considered $\$ 0.06$ per $\mathrm{kWh}$ electricity but included different financial assumptions. Both scenarios 5 and 6 had a low loan rate and a lower producer down payment requirement of $5 \%$. Scenario 5 had no heat recovery and scenario 6 had the same heat recovery as scenario 4 . Together these scenarios illustrate several of the more important economic and policy alternatives that could affect the future feasibility of anaerobic digestion of manures. Table 25 contains a more detailed description of the energy and economic scenarios, while table 26 details the other common model inputs.

Table 27 (Figures 10, 12, \& 13) notes energy production and system capital costs for the farrow to finish systems over the range of size modeled throughout the swine facility analysis. Tables 28 and 29 (Figures $5 \& 6$ ) show the economic feasibility, including net present value per sow (NPV) and payback period, for swine farrow to finish 
operations. Table 30 (Figures $11,12, \& 13$ ) notes energy production and system capital costs for swine finishing systems over the same range of facility sizes. Tables 31 and 32 (Figures $7 \& 8$ ) show the economic feasibility, including net present value per sow (NPV) and payback period, for swine finishing operations.

Table 33 (Figures 23 \& 28) note energy production and system capital costs for dairy tie stall facilities with plug flow digester systems. Tables 34 and 35 (Figures 14 \& 15) show the economic feasibility, including net present value per cow (NPV) and payback period, for dairy tie stall with plug flow digester operations over the range of sizes simulated. Table 36 (Figure 24) notes the energy production and system capital costs for the dairy tie stall with complete mix digester systems. Tables 37 and 38 (Figures 16 \& 17) show the economic feasibility, including net present value per cow (NPV) and payback period, for the dairy tie stall with complete mix digester operations.

Table 39 (Figures $25 \& 28$ ) notes the energy production and system capital costs for the dairy free stall with plug flow digester systems. Tables 40 and 41 (Figures 18 \& 19) below show the economic feasibility, including net present value per cow (NPV) and payback period, for the dairy free stall with plug flow digester operations over the range of sizes simulated. Table 42 (Figure 26) notes the energy production and system capital costs for the dairy free stall with complete mix digester systems. Tables 43 and 44 (Figures 20 \& 21) show the economic feasibility, including net present value per cow (NPV) and payback period, for the dairy free stall with complete mix digester operations.

System herd counts that met favorable economic guidelines are listed in table 45. For the farrow-to-finish operations, under all of the scenarios, the herd size had to be greater than 20,000 head for a methane project to be feasible. This is most likely attributed to the diluted manure inputs from the nursery and gestation facilities, which lowers the overall gas production of the system. With an average animal mass of $170 \mathrm{lbs}$. this translated to facilities with live weights greater than $3,400,000 \mathrm{lbs}$. From the information provided in the IDNR permit files, there were not currently any farrow-tofinish operations of this magnitude in lowa. The minimum economically feasible size for finishing operations was approximately 5,000 hogs (scenario \#3), at an animal weight of $135 \mathrm{lbs}$., and total live weight of $675,000 \mathrm{lbs}$. However, scenario \#3 assumed the rate for electricity doubles to $\$ 0.12$ per kilowatt. Data from IDNR indicates that approximately 179 of the permitted swine operations were this size or larger. The minimum economically feasible size for dairy operations was small enough to accommodate almost all of the permitted sites listed (scenario \#3). Even more of the sites would be feasible with scenario \#6, which considered the use of no-interest loans to encourage the installation of methane recovery facilities.

An economic scenario similar to scenario 1 but with an electricity rate of $\$ 0.08$ per kwh was run to find out how much a $30 \%$ rate increase would do to decrease herd sizes. For dairy operations more than 5,000 cows and for farrow-to-finish and finishing operations more than 20,000 sows or pigs were still needed to make anaerobic digestion economically feasible. An electricity rate of $\$ 0.10$ per $\mathrm{kwh}$, a $60 \%$ rate increase, was also simulated but not included in table 45 . The number of dairy cows to make anaerobic 
digestion feasible at $\$ 0.10$ per kwh was between 2,000 to 3,000 cows. For swine farrowto-finish operations the number of sows was still greater than 20,000 sows and for finishing operations the pigs required was between 10,000 to 15,000 pigs.

Figures $31 \& 32$ depict the maximum number of farms per county that have an average herd size that matches the herd size needed to be economically feasible in either scenario 3 or 6 (table 45). For scenario 6 Sioux County in northwest lowa has 18 farms eligible for scenario 3 and 4 farms feasible for scenario 6 based on the agricultural statistics data. For swine operations, central and northwestern lowa counties had significant number of farms eligible for scenario 6 . Only two counties in central Iowa, Hamilton and Hardin counties, had any farms that would be feasible for scenario \#3. While large farms appear necessary to achieve economies of scale in methane recovery, another possible solution is the use of centralized anaerobic digestion. Potential energy, MW of electricity output, from swine and dairy manure is graphically presented in Figures 29 \& 30, overlaid with the IDNR swine and dairy permitted sites. Centralized digestion facilities could be installed in counties with large livestock facilities that are closely located, or in counties with a high overall manure resource. One example would possibly have a digester installed in Sioux County, in northwest lowa, where there is a concentration of dairy facilities and a significant amount of manure from swine operations. An analysis of centralized digestion was not conducted since we did not have the proper models to consider the transportation of the waste, and the AgSTAR model simulations were stopped at limiting herd counts.

\section{Fossil Fuel Displacement}

One of the important questions for further policy development is the potential of biorenewable resources to displace current fossil fuel use. To address this question, the waste characterization and the economic analysis portions of the study have been combined to assess the energy that could be produced by methane recovery under each of the different scenarios. Table 47 lists a brief description of each policy scenario for fossil fuel displacement while table 48 lists the tons of coal or barrels of oil or cubic feet of natural gas that potentially could be displaced with livestock waste methane recovery. It has been estimated that Iowa consumed 1,140 trillion Btu of energy for year 2000 (IDNR, 2000 ). This is equivalent to $4.96 \times 10^{7}$ tons of coal, $1.94 \times 10^{8}$ barrels of $\# 2$ fuel oil, or $1.10 \times 10^{12}$ million cubic feet of natural gas/LNG. Total energy available from all of Iowa's manure resources would supply approximately $2.81 \%$ of Iowa's total present energy use from fossil fuel sources (policy scenario 1). If either scenario 5 or 6 were to become a reality, livestock methane energy recovery would displace $0.15 \%$ to $0.23 \%$ of Iowa's current total energy use from fossil fuel sources. Methane from livestock facilities has potential to displace a small fraction of the fossil fuel used for Iowa energy requirements.

\section{Market Assessment}

Another goal of this project was to identify roles, strategies, and follow-up steps for various stakeholder groups in encouraging implementation of anaerobic digestion and 
methane recovery technologies. A market assessment focus group meeting was held on June $21^{\text {st }}, 2000$ at the Madrid 4-H camp to discuss the barriers and opportunities to methane recovery from livestock facilities. Approximately 33 people attended the meeting, representing a wide range of organizations. Organizations represented included educational institutions, regulatory agencies, utilities, technology development and suppliers, policy analysts, nonprofit organizations, and farm operators/organizations. Table 46 notes some of the important issues, barriers, and opportunities that were identified by the meeting participants. A survey was also sent to swine and dairy producers asking them to rank these same issues dealing with methane recovery. The response was extremely poor from the swine producers so only dairy survey respondent information is included in this report. Only 2 out of 27 randomly selected swine operators responded ( $7 \%$ response) versus 8 out of 18 dairy operators responded ( $44 \%$ response).

The questionnaire sent out to the swine and dairy operators asked the producers details about their operations including animal numbers, waste production, waste storage, manure analysis information, energy usage (heat \& electricity), manure applied acreage, manure value, issues relating to anaerobic digestion, and had space for additional comments. Even though the survey answers were not always complete, the central theme that the producers noted about anaerobic digestion was the cost of the facilities. Although the survey response was not large enough to generate strong statistical results, it does indicate that dairy producers have some interest in anaerobic digestion and methane recovery, but are aware of the economic barriers. Addressing those economic barriers will be critical for widespread adoption of anaerobic digestion and methane recovery.

\section{Conclusions}

The State of lowa has a widely available manure resource distributed among a range of livestock types and manure management systems. The analyses described in this report show that anaerobic digestion with methane recovery is currently feasible in only limited circumstances on Iowa livestock operations. At the present time dairy and swine operations with scraped or liquid/slurry systems offer the most potential for methane capture. However, present economic conditions are not conducive to the installation of methane recovery facilities at swine or dairy operations. Economic incentives, such as doubling the electricity rate or providing no-interest loans, greatly lower the herd numbers needed to make methane recovery feasible. Other benefits such as odor control or bedding recovery could make anaerobic digestion more attractive to livestock producers, but are difficult to quantify.

Economics also proved to be a central theme for various stakeholders interested in methane recovery. Key issues include the low economic return on systems, the difficulties in obtaining financing for building systems, and the low rate paid for sale of excess electricity. Other important concerns were a lack of technical knowledge or assistance, and the complications interacting with utilities for interconnections. 
In light of the issues raised with this study, several conditions must be addressed if Iowa is to realize the potential for energy recovery from methane generated at livestock facilities. First, livestock producers and other stakeholders must be educated about the process of anaerobic digestion and methane recovery on livestock farms and its possible benefits and pitfalls. Dairy producers would be a logical initial focus of these educational efforts due to the response and comments on the survey. Second, the fundamental economics of anaerobic digestion need to improve, as the current energy price and policy structure is not at all encouraging for anaerobic digestion. Since some the possible benefits, such as odor control, are difficult to assign an economic value, other incentives such as low or no-interest loans, tax incentives, or grants may be necessary to encourage installation of systems. Third, utility companies will need to be very involved in any methane recovery program; so utility interconnection and other issues can be streamlined for both the livestock producer and utility company. Fourth, continued research and technology transfer efforts are needed to make anaerobic digestion and methane recovery a simpler, less management intensive, and more efficient process for the livestock producer or turnkey operator. Finally, centralized digestion with expert management should be explored as an option in areas with concentrated manure resources. A centralized system serving a number of satellite farms could encourage and demonstrate anaerobic digestion and methane recovery benefits, capturing the economies of scale of this technology for smaller and mid-sized livestock producers. While there is no single solution to the challenge of increasing methane recovery from livestock manure, a coordinated effort to implement these strategies would help Iowa realize the energy potential from this biorenewable resource.

\section{References}

APHIS. 1998. National Animal Health Monitoring System, Part III: Reference of 1997 Beef Cow-Calf Production Management and Disease Control. U.S. Department of Agriculture. Animal and Plant Health Inspection Service.

APHIS. 1996. National Animal Health Monitoring System, Part I: Reference of 1996 Dairy Management Practices. U.S. Department of Agriculture. Animal and Plant Health Inspection Service.

APHIS. 1996. National Animal Health Monitoring System, Part III: Reference of 1996 Dairy Health and Health Management. U.S. Department of Agriculture. Animal and Plant Health Inspection Service.

APHIS. 2000. National Animal Health Monitoring System, Part I: Baseline Reference of Feedlot Management Practices, 1999. U.S. Department of Agriculture. Animal and Plant Health Inspection Service.

APHIS. 1996. National Animal Health Monitoring System, Part III: Reference of 1996 Dairy Health and Health Management. U.S. Department of Agriculture. Animal and Plant Health Inspection Service. 
APHIS. 1996. National Animal Health Monitoring System, Part I: Reference of 1995 U.S. Grower/Finisher Health \& Management Practices. U.S. Department of Agriculture. Animal and Plant Health Inspection Service.

APHIS. 1995. National Animal Health Monitoring System, Part I: Reference of 1995 Swine Management Practices. U.S. Department of Agriculture. Animal and Plant Health Inspection Service.

APHIS. 2000. National Animal Health Monitoring System, Part II: Reference of 1999 Table Egg Layer Management in the U.S. U.S. Department of Agriculture. Animal and Plant Health Inspection Service.

ICP Consulting. 1999. Methods For Estimating Greenhouse Gas Emissions From Manure Management. Prepared Greenhouse Gas Committee, Emission Inventory Improvement Program.

Iowa State University. 2000. Hog Operation and Manure Management Survey. Department of Economics. B. Babcock ed. Ames, IA.

Iowa Department of Natural Resources (DNR). 2000. Iowa Energy Plan: 2000 Comprehensive Energy Plan Update. Energy and Geological Resources Division. Des Moines, IA.

Jewell, W. J., P. E. Wright, N. P. Fleszar, G. Green, A. Safinski, and A. Zucker. 1997. Evaluation of Anaerobic Digestion Options for Groups of Dairy Farms in Upstate New York. Cornell University. New York.

NEOS Corporation. 1994. Energy Conversion of Animal Manures Resource Inventory and Feasibility Analysis for Thirteen Western States. Prepared for Western Regional Biomass Energy Program (WRBEP).

United States Department of Agriculture; National Agricultural Statistics Service. 1999. 1997 - Iowa Census of Agriculture - State and County Data.

U. S. EPA. 1997. AgSTAR Handbook A Manual For Developing Biogas Systems at Commercial Farms in the United States. Edited by K. F. Roos and M. A. Moser. EPA 430-B97-015. Washington, D.C.

U. S. EPA. 1999. Feedlot Industry Sector Report Revised Draft Report. Preliminary Data Summary: Feedlots Point Source Category Study. EPA-821-R-99-002. 


\section{Appendix}

A 
Table 1: Summary of Potential Livestock Methane Energy in Iowa (based on county animal census data)

\begin{tabular}{|l|c|c|c|c|c|}
\hline Animal & $\begin{array}{c}\text { Animal } \\
\text { Numbers } \\
(\#)\end{array}$ & $\begin{array}{c}\text { Total } \\
\text { Solids } \\
\text { (tons/day) }\end{array}$ & $\begin{array}{c}\text { Volatile } \\
\text { Solids } \\
\text { (tons/yr) }\end{array}$ & $\begin{array}{c}\text { Collectable } \\
\text { Volatile Solids } \\
\text { (tons/yr) }\end{array}$ & $\begin{array}{c}\text { Potential } \\
\text { Energy } \\
\text { (MMBtu/yr) }\end{array}$ \\
\hline Feedlot Cattle & $3,306,618$ & 11,077 & $3,439,709$ & $2,579,782$ & $14,859,544$ \\
Dairy Cows & 222,142 & 1,555 & 454,058 & 408,652 & $2,402,876$ \\
Swine & $14,651,919$ & 10,989 & $3,208,770$ & $2,567,016$ & $13,348,484$ \\
Poultry Layers & $24,876,834$ & 808 & 217,921 & 196,129 & $1,214,431$ \\
Poultry Boilers & $1,017,224$ & 22 & 5,941 & 5,347 & 33,106 \\
Turkeys & $2,552,624$ & 217 & 60,561 & 36,337 & 224,996 \\
\hline Totals & & 24,668 & $7,386,961$ & $5,793,263$ & $32,083,438$ \\
\hline
\end{tabular}

Table 2: Iowa Department of Natural Resources Permitted Facility Waste Management Profile

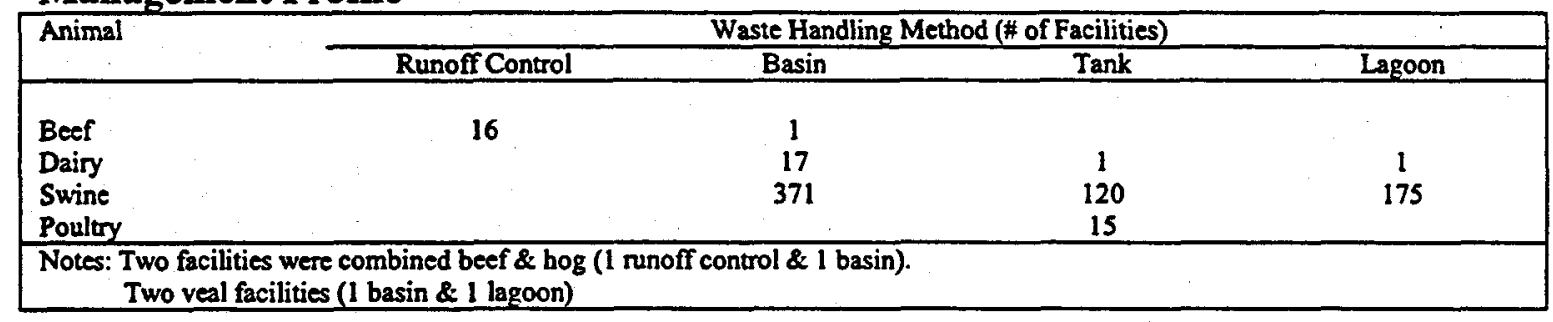

Table 3: EPA Waste Handling and Management for Livestock Types in Iowa

\begin{tabular}{|l|c|c|c|c|c|c|c|c|c|}
\hline Animal & $\begin{array}{c}\text { Anaerobic } \\
\text { Lagoon } \\
\%^{1}\end{array}$ & $\begin{array}{c}\text { Dry Lot } \\
\%\end{array}$ & $\begin{array}{c}\text { Liquid/ } \\
\text { Slurty } \\
\%\end{array}$ & $\begin{array}{c}\text { Pasture } \\
\%\end{array}$ & $\begin{array}{c}\text { Daily } \\
\text { Spread } \\
\%\end{array}$ & $\begin{array}{c}\text { Solid } \\
\text { Storage } \\
\%\end{array}$ & $\begin{array}{c}\text { Pit } \\
\text { Storage } \\
\%\end{array}$ & $\begin{array}{c}\text { Litter } \\
\%\end{array}$ & $\begin{array}{c}\text { Other } \\
\%\end{array}$ \\
\hline $\begin{array}{l}\text { Beef } \\
\text { Dairy }\end{array}$ & 3 & 13 & & 87 & & & & \\
Swine & 3 & 30 & 20 & & 8 & 65 & & 4 \\
Layers & 2 & 4 & & & 50 & 50 & 13 \\
Boilers & 2 & & & & & 100 & 4 \\
Turkeys & & & & & & & & 100 \\
\hline
\end{tabular}

Source: EPA. Feedlot Industry Sector Profile Revised Draft Report. 1998

'all percentages expressed here are based on operation totals.

the total swine operations only add up to $96 \%$, this is an error in the source material

Table 4: Swine Herd Percentages and Waste Management Strategies

\begin{tabular}{|c|c|c|c|c|c|c|}
\hline \multirow[t]{2}{*}{ Herd Category } & \multirow{2}{*}{$\begin{array}{c}\text { Overall \% of } \\
\text { Hog \#'s }\end{array}$} & \multicolumn{5}{|c|}{ Waste Management System (\% of Pigs within Herd Category) } \\
\hline & & Solid Manure & Deep Pit & Earthen Basin & Formed Storage & Lagoon \\
\hline $0-250$ & 2.79 & 86.7 & 8.6 & 1.1 & 3.3 & 0 \\
\hline $251-500$ & 10.17 & 69.9 & 23.1 & 1.2 & 5.6 & 0 \\
\hline $501-1000$ & 17.14 & 56.3 & 29.3 & 2.4 & 5.4 & 6.6 \\
\hline $1001-2000$ & $69.9 *$ & N/A & 21.4 & 1.7 & 4.3 & 2.2 \\
\hline $2001-4000$ & & $\mathbf{N} / \mathbf{A}$ & 16.6 & 1.7 & 5.2 & 4.4 \\
\hline 4001 or more & $\therefore$ & N/A & 21.1 & 3.0 & 9.1 & 9.0 \\
\hline
\end{tabular}

Covers 1001-2000,20014000, and 4001 or more categories

Source: Iowa State University Hog Operation and Management Survey, 2000 
Table 5: Feedlot Manure Disposal Based on Percentage of Operations and Cows

\begin{tabular}{|lcc|}
\hline Manure Disposal Method & \% of Operations & \% of Beef Cows \\
\hline Drag or harrow pastures & 43.0 & 44.2 \\
Hauled and spread onto land used for grazing or forage production for & & 34.1 \\
operation & 25.5 & 25.4 \\
Hauled and spread onto other land & 21.9 & 1.7 \\
Other & 2.5 & 32.4 \\
No disposal & 34.7 & . \\
\hline
\end{tabular}

Source: APHIS. 1998. National Animal Health Monitoring System. Part III: Reference of 1997 Beef Cow-Calf Production Management and Disease Control. U. S. Department of Agriculture, Animal and Plant Health Inspection Service.

Table 6: Cow-Calf Operations Manure Disposal Methods by \% of Operations

\begin{tabular}{|lccc|}
\hline & & \% of Operations & \\
\cline { 3 - 4 } Manure Disposal Method & $1,000-7,999$ & 8,000 or more & All Operations \\
\hline & & & \\
Applied on land owned or managed by the & 90.9 & 61.7 & 82.9 \\
feedlot & 5.0 & 26.7 & 11.0 \\
Sold & 15.1 & 57.3 & 26.7 \\
Given away & 0.8 & 9.9 & 3.3 \\
Removed by paying someone to take it & 2.7 & 5.2 & 3.4 \\
Removed by another method & & & \\
\hline
\end{tabular}

Source: APHIS. 1999. National Animal Health Monitoring System. Part I: Baseline Reference of Feedlot Management Practices, 1999. U. S. Department of Agriculture, Animal and Plant Health Inspection Service.

Table 7: Cow-Calf Operations Manure Disposal Methods by \% of Manure

\begin{tabular}{|lccc|}
\hline & & $\%$ of Manure & All Operations \\
\cline { 2 - 4 } Manure Disposal Method & $1,000-7,999$ & 8.000 or more & \\
\hline Applied on land owned or managed by the & & & 33.4 \\
feedlot & 74.6 & 25.5 & 13.2 \\
Sold & 4.8 & 14.8 & 43.3 \\
Given away & 16.2 & 48.5 & 7.3 \\
Removed by paying someone to take it & 1.9 & 8.4 & 2.8 \\
Removed by another method & 2.5 & 2.8 & \\
\hline
\end{tabular}

Source: APHIS. 1999. National Animal Health Monitoring System. Part l: Baseline Reference of Feedlot Management Practices,

1999. U. S. Department of Agriculture, Animal and Plant Health Inspection Service.

Table 8: Dairy Housing Systems Based on Animal Types

\begin{tabular}{|c|c|c|c|c|}
\hline \multirow[b]{2}{*}{ Housing Type } & \multicolumn{4}{|c|}{$\%$ of Operations } \\
\hline & $\begin{array}{c}\text { Unweaned Dairy } \\
\text { Heifers }\end{array}$ & $\begin{array}{c}\text { Weaned Dairy } \\
\text { Heifers }\end{array}$ & $\begin{array}{l}\text { Lactating Dainy } \\
\text { Cows }\end{array}$ & Maternity \\
\hline $\begin{array}{l}\text { Freestall } \\
\text { Individual animal area } \\
\text { Multiple animal area } \\
\text { Tie stall or stanchion } \\
\text { Drylot } \\
\text { Pasture } \\
\text { Hutch }\end{array}$ & $\begin{array}{l}2.5 \\
29.7 \\
40.0 \\
10.5 \\
9.1 \\
7.4 \\
32.5\end{array}$ & $\begin{array}{l}9.7 \\
6.6 \\
73.9 \\
11.5 \\
38.1 \\
51.4 \\
\text { N/A }\end{array}$ & $\begin{array}{l}24.4 \\
2.3 \\
17.9 \\
61.4 \\
47.2 \\
59.6 \\
\text { N/A }\end{array}$ & $\begin{array}{l}5.6 \\
38.3 \\
26.3 \\
26.3 \\
28.9 \\
41.9 \\
\text { N/A }\end{array}$ \\
\hline
\end{tabular}

Source: APHIS. 1996. National Animal Health Monitoring System. Part I: Reference of 1996 Dairy Management Practices. U. S. Department of Agriculture, Animal and Plant Health Inspection Service.

Table 9: Dairy Milking Facility Type Based on \% of Operations and Cows

\begin{tabular}{|lcc|}
\hline Milking Facility Type & \% of Operations & \% of Cows \\
\hline Parlor & 28.8 & 54.9 \\
Tie stall or stanchion & 69.5 & 43.9 \\
Other & 2.9 & 4.3 \\
\hline
\end{tabular}

Source: APHIS. 1996. National Animal Health Monitoring System. Part I: Reference of 1996 Dainy Management Practices. U. S. Department of Agriculture, Animal and Plant Health Inspection Service. 
Table 10: Type of Dairy Manure Collection System

\begin{tabular}{|lcccc|}
\hline & \multicolumn{4}{c|}{ \% of Operations } \\
\cline { 2 - 5 } Method & Less than 100 & \multicolumn{2}{c|}{ Number of Dairy Cows } \\
$100-199$ & 200 or more & Total \\
\hline Gutter scraper & 74.0 & 34.7 & 9.0 & 63.2 \\
Alley scraper & 50.2 & 82.4 & 85.0 & 57.7 \\
(Mechanical or Tractor) & & & 26.6 & 2.8 \\
Water flushed alley & 0.2 & 4.3 & 0.1 & 1.1 \\
Other & 1.3 & 0.5 & \\
\hline
\end{tabular}

Source: APHIS. 1996. National Animal Health Monitoring System. Part III: Reference of 1996 Dairy Health and Health

Management. U. S. Department of Agriculture, Animal and Plant Health Inspection Service.

Table 11: Type of Dairy Manure Management or Storage System

\begin{tabular}{|c|c|c|c|c|}
\hline \multirow[b]{3}{*}{ Waste System } & \multicolumn{4}{|c|}{$\%$ of Operations } \\
\hline & \multicolumn{4}{|c|}{ Number of Dairy Cows } \\
\hline & Less than 100 & $100-199$ & 200 or more & Total \\
\hline Below floor shurry or deep pit & 4.7 & 19.6 & 16.9 & 7.9 \\
\hline Slurry storage in tanks & 3.2 & 10.9 & 17.5 & 5.4 \\
\hline Slurry storage in earth basin & 13.5 & 25.2 & 27.8 & 16.3 \\
\hline Anacrobic lagoon w/cover & 0.2 & 0.3 & 1.1 & 0.3 \\
\hline Anaerobic lagoon w/o cover & 5.7 & 18.2 & 46.7 & 10.7 \\
\hline Aerated Lagoon & 0.5 & 3.3 & 8.3 & 1.5 \\
\hline Manure Pack (Inside bam) & 22.3 & 20.4 & 14.1 & 21.4 \\
\hline Outside storage for solids & & & & \\
\hline (not in drylot or pen) & 37.9 & 33.0 & 30.1 & 36.6 \\
\hline Outside storage in drylot or pens & 14.8 & 11.5 & 22.0 & 14.9 \\
\hline Solids in building w/o cattle access & 2.8 & 4.1 & 2.4 & 3.0 \\
\hline Other & 2.1 & 1.8 & 1.6 & 2.0 \\
\hline
\end{tabular}

Source: APHIS. 1996. National Animal Health Monitoring System. Part III: Reference of 1996 Dairy Health and Health Management. U.S. Department of Agriculture, Animal and Plant Health Inspection Service.

Table 12: \% of Dairy Lagoon Systems with Single or Multiple Stages

\begin{tabular}{|lc|}
\hline Stages & \% of Operations \\
\hline Single stage & 84.0 \\
Multiple stage & 16.6 \\
\hline
\end{tabular}

Source: APHIS. 1996. National Animal Health Monitoring System. Part III: Reference of 1996 Dainy Health and Health

Management. U.S. Department of Agriculture, Animal and Plant Health Inspection Service.

Table 13: \% of Dairy Lagoons or Tank Storages with Solid Separation

\begin{tabular}{|lc|}
\hline Response & \% of Operations \\
\hline Yes & 2.3 \\
No & 29.5 \\
No tank or lagoon storage & 68.2 \\
\hline
\end{tabular}

Source: APHIS. 1996. National Animal Health Monitoring System. Pant III: Reference of 1996 Dairy Health and Health

Manogement. U. S. Department of Agriculture, Animal and Plant Health Inspection Service.

\section{Table 14: Dairy Manure Disposal Method}

\begin{tabular}{|c|c|c|c|c|}
\hline \multirow{2}{*}{ Method } & \multicolumn{4}{|c|}{$\%$ of Operations } \\
\hline & Less than 100 & $\begin{array}{l}100-199 \\
100\end{array}$ & 200 or more & Total \\
\hline $\begin{array}{l}\text { Applied on land owned or rented by the operation } \\
\text { Sold or received other compensation } \\
\text { Gave away } \\
\text { Composted }\end{array}$ & $\begin{array}{l}99.4 \\
0.8 \\
4.9 \\
3.3\end{array}$ & $\begin{array}{l}98.6 \\
2.7 \\
8.7 \\
6.2 \\
\end{array}$ & $\begin{array}{l}94.7 \\
17.5 \\
22.6 \\
16.3\end{array}$ & $\begin{array}{l}98.9 \\
2.3 \\
6.8 \\
4.7\end{array}$ \\
\hline
\end{tabular}

Source: APHIS. 1996. National Animal Health Monitoring System. Part III: Reference of 1996 Dainy Health and Health Management. U.S. Department of Agriculture, Animal and Plant Health Inspection Service. 
Table 15: Frequency of Dairy Manure Application

\begin{tabular}{|lcc|}
\hline & & \% of Operations \\
\cline { 2 - 3 } Frequency & Summer & Winter \\
\hline Daily & & 46.5 \\
Weekly & 30.4 & 14.7 \\
Monthly & 25.5 & 7.8 \\
Less than monthly & 10.8 & 31.0 \\
\hline
\end{tabular}

Source: APHIS. 1996. National Animal Health Monitoring System. Part III: Reference of 1996 Dainy Health and Health Management. U. S. Department of Agriculture, Animal and Plant Health Inspection Service.

Table 16: Dairy Manure Application Method

\begin{tabular}{|c|c|c|c|c|}
\hline \multirow[b]{3}{*}{ Application Method } & \multicolumn{4}{|c|}{$\%$ of Operations } \\
\hline & & Number of I & & \\
\hline & Less than 100 & $100-199$ & 200 or more & Total \\
\hline Irigation & 3.1 & 11.2 & 40.5 & 7.0 \\
\hline Broadcast/solid spreader & 90.6 & 85.1 & 75.8 & 88.7 \\
\hline Slurry (surface application) & 17.7 & 38.5 & 44.6 & 22.7 \\
\hline Sluny (subsurface injection) & 3.6 & 5.9 & 8.6 & 4.3 \\
\hline Other methods & 0.0 & 0.4 & 1.0 & 0.1 \\
\hline
\end{tabular}

Source: APHIS. 1996. National Animal Health Monitoring System. Part III: Reference of 1996 Dairy Health and Health

Management. U.S. Department of Agriculture, Animal and Plant Health Inspection Service.

Table 17: Dairy Manure Specific Management Practices

\begin{tabular}{|c|c|c|c|c|}
\hline \multirow[b]{3}{*}{ Management Practice } & \multicolumn{4}{|c|}{$\%$ of Operations } \\
\hline & \multicolumn{3}{|c|}{ Number of Dairy Cows } & \multirow[b]{2}{*}{ Total } \\
\hline & Less than 100 & $100-199$ & 200 or more & \\
\hline Analyzed nutrient content of the manure & 10.3 & 26.4 & 28.6 & 14.0 \\
\hline $\begin{array}{l}\text { Established manure application rate based on } \\
\text { manure nutrient or crop need }\end{array}$ & 41.7 & 50.5 & 43.3 & 43.2 \\
\hline Applied manure a minimum of $50 \mathrm{ft}$ from a water & & & & \\
\hline $\begin{array}{l}\text { body } \\
\text { Incorporated manure into soil within } 24 \text { hours }\end{array}$ & 77.9 & 77.2 & 82.5 & 78.1 \\
\hline after application & 15.8 & 22.8 & 31.5 & 17.9 \\
\hline
\end{tabular}

Table 18: Production Phases Within Swine Operations

\begin{tabular}{|lc|}
\hline Production Phase & \% of Operations \\
\hline Farrowing & \\
Nursery & 70.7 \\
Grower/Finisher & 46.5 \\
\hline
\end{tabular}

Source: APHIS. 1995. National Animal Health Monitoring Syssem. Part I: Reference of 1995 Swine Management Practices. U.S.

Department of Agriculture, Animal and Plant Health Inspection Service.

Table 19: Facility Types in Each Production Phase by \% of Operations and Hogs

\begin{tabular}{|c|c|c|c|c|c|c|}
\hline & \multicolumn{3}{|c|}{$\%$ of Operations } & \multicolumn{3}{|c|}{$\%$ of Pigs } \\
\hline Facility Type & Farrow & Nursery & $\begin{array}{l}\text { Grower' } \\
\text { Finisher }\end{array}$ & Farrow & Nursery & $\begin{array}{l}\text { Grower/ } \\
\text { Finisher }\end{array}$ \\
\hline $\begin{array}{l}\text { Total confinement } \\
\text { Open building with no outside } \\
\text { access - } \\
\text { Open building with outside access } \\
\text { Lot with hut or no building } \\
\text { Pasture with hut or no building }\end{array}$ & $\begin{array}{c}9.8 \\
30.5 \\
5.7 \\
7.6\end{array}$ & $\begin{array}{c}9.9 \\
26.1 \\
3.0 \\
1.5\end{array}$ & $\begin{array}{l}11.9 \\
45.5 \\
9.9 \\
6.7\end{array}$ & $\begin{array}{l}5.9 \\
9.7 \\
1.6 \\
1.5\end{array}$ & $\begin{array}{l}87.2 \\
5.8 \\
5.6 \\
1.1 \\
0.3\end{array}$ & $\begin{array}{l}56.5 \\
11.3 \\
27.8 \\
2.9 \\
1.5\end{array}$ \\
\hline
\end{tabular}

Source: APHIS. 1995. National Animal Health Monitoring System. Part I: Reference of 1995 Swine Management Practices. U.S. Department of Agriculture, Animal and Plant Health Inspection Service. 
Table 20: Type of Grower/Finisher Facility Waste Handling System

\begin{tabular}{|lccc|}
\hline & Farrowing & \% of Operations in Growth Category \\
System & & & Grower/Finisher \\
\hline & 13.8 & 4.3 & 14.8 \\
None & 25.5 & 33.7 & 23.2 \\
Pit-holding & 12.0 & 17.6 & 24.9 \\
Mechanical scraper/tractor & 38.2 & 29.9 & 27.2 \\
Hand cleaned & 5.3 & 9.4 & 2.4 \\
Fushed-under slats & 3.0 & 2.1 & 3.4 \\
Flushed-open gutter & 2.2 & 3.0 & 4.1 \\
Other & & & \\
\hline
\end{tabular}

Source: APHIS. 1995 National Animal Health Moniloring System, Part II: Reference of 1995 U.S. Grower/Finisher Health \& Management Practices. U.S. Department of Agriculture, Animal and Plant Health Inspection Service.

Table 21: Grower/Finisher Operation Flooring Types

\begin{tabular}{|c|c|c|}
\hline Flooring Types & $\%$ of Operations & $\%$ of Hogs \\
\hline Concrete slats only & 26.3 & 38.8 \\
\hline Metal slats only & 3.6 & 0.8 \\
\hline Fiberglass or plastic slats only & 2.7 & 0.8 \\
\hline Slats and other flooring combined (partial slats) & 33.0 & 23.9 \\
\hline Solid concrete only & 61.6 & 31.4 \\
\hline Dirt/pasture only & 12.1 & 3.2 \\
\hline Wood only & 0.0 & 0.0 \\
\hline Other & 1.9 & 1.1 \\
\hline
\end{tabular}

Source: APHIS. 1995 National Animal Health Monitoring System, Part II: Reference of 1995 U.S. Grower/Finisher Health \& Management Practices. U.S. Department of Agriculture, Animal and Plant Health Inspection Service.

Table 22: Grower/Finisher Waste Storage Systems

\begin{tabular}{|l|c|c|c|c|}
\hline System & $\begin{array}{c}\text { All Operations } \\
(\%)\end{array}$ & $\begin{array}{c}\text { Less than 2,000 } \\
\text { head }\end{array}$ & $\begin{array}{c}2,000-9,999 \text { head } \\
10,000 \text { or more } \\
\text { head }\end{array}$ \\
\hline Below floor slurry or deep pit & 49.9 & 43.6 & 70.4 & 47.9 \\
Above ground slurry storage & 5.6 & 4.1 & 10.3 & 8.3 \\
Below ground slurry storage & 19.4 & 17.3 & 25.6 & 26.8 \\
Anaerobic lagoon with cover & 1.8 & 2.2 & 0.5 & 2.0 \\
Anaerobic lagoon without cover & 20.9 & 17.4 & 29.2 & 81.8 \\
Aerated lagoon & 2.6 & 1.3 & 6.9 & 1.0 \\
Oxidation ditch & 2.2 & 2.9 & 0.1 & 0.0 \\
Solids separated from liquids & 4.6 & 4.1 & 5.9 & 4.7 \\
Other & 0.4 & 0.6 & 0.0 & 1.1 \\
\hline
\end{tabular}

Source: APHIS. 1995 National Animal Health Monitoring System. Part II: Reference of 1995 U.S. Grower/Finisher Health \& Management Practices. U.S. Department of Agriculture, Animal and Plant Health Inspection Service.

\section{Table 23: Grower/Finisher Operations Disposing of Liquid/Solid Waste or} Unseparated Waste

\begin{tabular}{|l|c|}
\hline Waste Type & \% of Operations \\
\hline Separated liquids and solids & 4.3 \\
Unseparated liquids and solids & 96.2 \\
\hline
\end{tabular}

Source: APHIS. 1995 National Animal Health Monitoring System. Part II: Reference of 1995 U.S. Grower/Finisher Health \&

Maragement Practices. U.S. Department of Agriculture, Animal and Plant Health Inspection Service. 
Table 24: Manure Handling Methods for Layer Operations in the Central U. S.

\begin{tabular}{|l|c|}
\hline Primary Manure Handling Method & \% of Farm Sites in the Central \\
\hline & U. S. \\
High Rise (pit at ground level with house above) & 48.1 \\
Deep Pit (below ground) & 6.4 \\
Shallow Pit (pit at ground level with raised cages) & 1.6 \\
Flush System to a Lagoon & 0.0 \\
Manure Belt & 20.2 \\
Scraper System (not thush) & 23.7 \\
\hline
\end{tabular}

Source: APHIS. 1999 National Animal Health Monitoring System, Part II: Reference of 1999 U.S. Table Egg Layer Management in the U.S. U.S. Department of Agriculture, Animal and Plant Health Inspection Service.

Table 25: AgSTAR Model Scenario Descriptions

\begin{tabular}{|c|c|c|c|c|}
\hline $\begin{array}{c}\text { Scenario } \\
\text { (\$) }\end{array}$ & $\begin{array}{c}\text { Electricity } \\
\text { Rate } \\
(\text { S/kwh) }\end{array}$ & $\begin{array}{c}\text { Liquid Propane } \\
\text { (\% heat supp.) } \\
\text { \& (S/gallon) }\end{array}$ & $\begin{array}{c}\text { Loan Rate } \\
\%\end{array}$ & $\begin{array}{c}\text { Producer } \\
\text { Downpayment } \\
\%\end{array}$ \\
\hline 1 & 0.06 & 0 & 10 & 20 \\
2 & 0.08 & 0 & 10 & 20 \\
3 & 0.12 & 0 & 10 & 20 \\
4 & 0.06 & $90 \& 1.00$ & 10 & 20 \\
5 & 0.06 & 0 & 0 & 5 \\
6 & 0.06 & $90 \& 1.00$ & 0 & 5 \\
\hline
\end{tabular}

Table 26: Other AgSTAR Model Input Values

\begin{tabular}{|c|c|}
\hline Model Parameter & Input or Assumed Value \\
\hline ClimatelLocation & Story County, IA \\
Livestock Systems & Swine Farrow to Finish \& Finishing \\
Dairy Free \& Tie Stall \\
Waste Collection Method & Swine - Plug Pull or Cascade Dam \\
Dairy - Flush Parlor and Scrape Rest & Storage Tank \\
Storage System & Swine - Complete Mix \\
Digester Type & Dairy - Complete Mix or Phug Flow \\
Other Benefits & None \\
Any Other Model Values & AgSTAR Default Values \\
\hline
\end{tabular}

Table 27: Swine Farrow to Finish System Energy Production and Costs

\begin{tabular}{|c|c|c|c|c|c|c|c|}
\hline Herd & $\begin{array}{c}\text { Total } \\
\text { Manure } \\
\text { (gal/d) }\end{array}$ & $\begin{array}{c}\text { Gas } \\
\text { Production } \\
\text { (cu fud) }\end{array}$ & $\begin{array}{c}\text { Max. } \\
\text { Energy } \\
\text { (kwh) }\end{array}$ & $\begin{array}{c}\text { Mix } \\
\text { Tank } \\
(\boldsymbol{S})\end{array}$ & $\begin{array}{c}\text { Complete } \\
\text { Mix Digester } \\
(\mathbf{S})\end{array}$ & $\begin{array}{c}\text { Generator } \\
\text { Building } \\
(\mathbf{S})\end{array}$ & $\begin{array}{c}\text { Total } \\
\text { Cost } \\
(\boldsymbol{S})\end{array}$ \\
\hline 50 & 503 & 1,298 & 33,580 & 17,260 & 33,007 & 19,025 & 94,292 \\
100 & 1,011 & 2,608 & 67,890 & 19,133 & 47,578 & 23,138 & 114,848 \\
200 & 1,980 & 5,111 & 133,225 & 21,863 & 72,402 & 30,969 & 150,233 \\
400 & 4,021 & 10,378 & 270,465 & 26,293 & 119,781 & 47,419 & 218,493 \\
600 & 6,067 & 15,654 & 408,070 & 29,997 & 164,611 & 63,912 & 283,520 \\
800 & 8,138 & 20,998 & 547,135 & 33,368 & 208,685 & 80,581 & 347,634 \\
1,000 & 10,203 & 26,322 & 686,200 & 36,481 & 251,707 & 97,250 & 410,437 \\
1,500 & 15,209 & 39,241 & $1,022,730$ & 43,408 & 353,957 & 137,588 & 559,953 \\
2,000 & 20,405 & 52,645 & $1,372,400$ & 49,974 & 457,563 & 179,500 & 712,037 \\
3,000 & 30,566 & 78,860 & $2,055,680$ & 61,825 & 656,982 & 261,400 & $1,005,207$ \\
5,000 & 51,109 & 131,854 & $3,093,812$ & 83,544 & $1,051,702$ & 427,038 & $1,587,284$ \\
10,000 & 102,272 & 263,845 & $6,878,790$ & 132,085 & $2,015,225$ & 839,512 & $3,011,822$ \\
15,000 & 153,472 & 395,931 & $9,125,000$ & 177,066 & $2,966,579$ & $1,110,750$ & $4,277,396$ \\
20,000 & 204,635 & 527,921 & $9,125,000$ & 220,152 & $3,910,481$ & $1,108,750$ & $5,264,383$ \\
\hline
\end{tabular}

only sow numbers, used default AGSTAR values for Nursery, Grower, Finisher, and Boar numbers.

- includes sow, nursery, grower, finisher, and boar manure production 
Table 28: Swine Farrow to Finish System Methane Recovery Economics

\begin{tabular}{|c|c|c|c|c|c|c|}
\hline \multirow{2}{*}{ Herd } & \multicolumn{2}{|c|}{ Scenario \#1 } & \multicolumn{2}{c|}{ Scenario \#2 } & \multicolumn{2}{c|}{ Scenario \#3 } \\
\cline { 2 - 7 }$(\#)$ & $\begin{array}{c}\text { NPV } \\
\text { (S/sow) }\end{array}$ & $\begin{array}{c}\text { Payback } \\
\text { (years) }\end{array}$ & $\begin{array}{c}\text { NPV } \\
\text { (S/sow) }\end{array}$ & $\begin{array}{c}\text { Payback } \\
\text { (years) }\end{array}$ & $\begin{array}{c}\text { NPV } \\
\text { (S/sow) }\end{array}$ & $\begin{array}{c}\text { Payback } \\
\text { (years) }\end{array}$ \\
\hline 50 & $-1,436.96$ & 69.3 & -1359.00 & 48.0 & -1203.10 & 29.7 \\
100 & -820.74 & 43.8 & -744.71 & 30.2 & -592.66 & 18.7 \\
200 & -521.34 & 36.6 & -457.87 & 24.7 & -330.95 & 15.0 \\
400 & -380.90 & 35.2 & -327.94 & 23.0 & -222.02 & 13.6 \\
600 & -329.69 & 34.2 & -280.27 & 22.0 & -181.41 & 12.8 \\
800 & -303.34 & 33.6 & -255.68 & 21.4 & -160.35 & 12.4 \\
1,000 & -286.42 & 33.1 & -239.82 & 20.9 & -146.61 & 12.0 \\
1,500 & -258.78 & 31.6 & -213.59 & 19.8 & -123.20 & 11.4 \\
2,000 & -246.81 & 31.1 & -202.32 & 19.4 & 113.35 & 11.1 \\
3,000 & -231.12 & 30.1 & -187.34 & 18.7 & -99.78 & 10.6 \\
5,000 & -218.20 & 29.3 & -174.98 & 18.1 & -88.55 & 10.3 \\
10,000 & -205.76 & 28.4 & -162.97 & 17.5 & -77.39 & 9.9 \\
15,000 & -183.43 & 24.5 & -140.78 & 15.6 & -55.49 & 9.1 \\
20,000 & -147.52 & 19.3 & -104.95 & 13.0 & -19.82 & 7.9 \\
\hline
\end{tabular}

Table 29: Swine Farrow to Finish System Methane Recovery Economics

\begin{tabular}{|c|c|c|c|c|c|c|}
\hline \multirow{2}{*}{ Herd } & \multicolumn{2}{|c|}{ Scenario \#4 } & \multicolumn{2}{c|}{ Scenario \#5 } & \multicolumn{2}{c|}{ Scenario \#6 } \\
\cline { 2 - 7 } & $\begin{array}{c}\text { NPV } \\
\text { (S/sow) }\end{array}$ & $\begin{array}{c}\text { Payback } \\
\text { (years) }\end{array}$ & $\begin{array}{c}\text { NPV } \\
\text { (\$/sow) }\end{array}$ & $\begin{array}{c}\text { Payback } \\
\text { (years) }\end{array}$ & $\begin{array}{c}\text { NPV } \\
\text { (S/sow) }\end{array}$ & $\begin{array}{c}\text { Payback } \\
\text { (years) }\end{array}$ \\
\hline 50 & -1407.62 & 59.4 & -698.32 & 69.3 & -668.98 & 59.4 \\
100 & -791.40 & 37.3 & -370.91 & 43.8 & -341.57 & 37.3 \\
200 & -491.99 & 29.9 & -227.27 & 36.6 & -197.78 & 29.9 \\
400 & -351.56 & 27.2 & -166.95 & 35.2 & -137.61 & 27.2 \\
600 & -300.35 & 25.7 & -144.61 & 34.2 & -115.27 & 25.7 \\
800 & $-274,00$ & 24.8 & -133.14 & 33.6 & -103.80 & 24.8 \\
1,000 & -257.08 & 24.2 & -125.66 & 33.1 & -96.32 & 24.2 \\
1,500 & -229.44 & 22.8 & -112.57 & 31.6 & -83.23 & 22.8 \\
2,000 & -217.47 & 22.3 & -107.36 & 31.1 & -78.02 & 22.3 \\
3,000 & -201.77 & 21.4 & -99.88 & 30.1 & -70.54 & 21.4 \\
5,000 & -188.86 & 20.7 & -93.86 & 29.3 & -64.52 & 20.7 \\
10,000 & -176.42 & 19.9 & -87.80 & 28.4 & -58.46 & 19.9 \\
15,000 & -154.09 & 17.6 & -71.74 & 24.5 & -42.40 & 17.6 \\
20,000 & -118.18 & 14.5 & -44.42 & 19.3 & -15.08 & 14.5 \\
\hline
\end{tabular}

Table 30: Finishing System Energy Production and Costs

\begin{tabular}{|c|c|c|c|c|c|c|c|}
\hline $\begin{array}{l}\text { Herd } \\
\text { (\#) }\end{array}$ & $\begin{array}{l}\text { Total } \\
\text { Manure } \\
\text { (gal/d) }\end{array}$ & $\begin{array}{l}\text { Gas } \\
\text { Production } \\
\text { (cu ftd) }\end{array}$ & $\begin{array}{l}\text { Max. } \\
\text { Energy } \\
\text { (kwh) }\end{array}$ & $\begin{array}{l}\text { Mix } \cdots \\
\text { Tank } \\
(\$)\end{array}$ & $\begin{array}{l}\text { Complete } \\
\text { Mix Digester } \\
\text { (S) }\end{array}$ & $\begin{array}{l}\text { Generator } \\
\text { Building } \\
\text { (S) }\end{array}$ & $\begin{array}{l}\text { Total } \\
\text { Cost } \\
(\$)\end{array}$ \\
\hline $\begin{array}{c}50 \\
100 \\
200 \\
400 \\
600 \\
800 \\
1,000 \\
1,500 \\
2,000 \\
3,000 \\
5,000 \\
10,000 \\
15,000 \\
20,000\end{array}$ & $\begin{array}{c}69 \\
137 \\
275 \\
550 \\
825 \\
1,100 \\
1,375 \\
2,062 \\
2,750 \\
4,125 \\
6,875 \\
13,749 \\
20,624 \\
27,499 \\
\end{array}$ & $\begin{array}{c}175 \\
350 \\
700 \\
1,400 \\
2,100 \\
2,799 \\
3,499 \\
5,249 \\
6,998 \\
10,498 \\
17,496 \\
34,992 \\
52,488 \\
69,984\end{array}$ & $\begin{array}{c}4,380 \\
8,760 \\
17,885 \\
36,135 \\
54,385 \\
72,635 \\
90,885 \\
136,510 \\
182,135 \\
273,385 \\
455,885 \\
912,135 \\
1,368,385 \\
1,824,270\end{array}$ & $\begin{array}{l}14,591 \\
15,174 \\
16,022 \\
17,270 \\
18,265 \\
19,130 \\
19,911 \\
21,636 \\
23,153 \\
25,831 \\
30,415 \\
39,922 \\
48,167 \\
55,759\end{array}$ & $\begin{array}{l}16,623 \\
19,694 \\
24,692 \\
33,074 \\
40,549 \\
47,553 \\
54,258 \\
70,195 \\
85,389 \\
144,900 \\
169,921 \\
301,472 \\
428,485 \\
553,150\end{array}$ & $\begin{array}{r}15,525 \\
-16,050 \\
17,144 \\
19,331 \\
21,519 \\
23,706 \\
25,894 \\
31,362 \\
36,831 \\
47,769 \\
69,644 \\
124,331 \\
179,019 \\
233,662\end{array}$ & $\begin{array}{l}71,739 \\
75,917 \\
82,858 \\
94,675 \\
105,333 \\
115,390 \\
125,063 \\
148,193 \\
170,373 \\
213,090 \\
294,980 \\
490,725 \\
680,671 \\
867,571\end{array}$ \\
\hline
\end{tabular}


Table 31: Finishing System Methane Recovery Economics

\begin{tabular}{|c|c|c|c|c|c|c|}
\hline \multirow{2}{*}{ Herd } & \multicolumn{2}{|c|}{ Scenario \#1 } & \multicolumn{2}{c|}{ Scenario \#2 } & \multicolumn{2}{c|}{ Scenario \#3 } \\
\cline { 2 - 7 } & $\begin{array}{c}\text { NPV } \\
\text { (S/pig) }\end{array}$ & $\begin{array}{c}\text { Payback } \\
\text { (years) }\end{array}$ & $\begin{array}{c}\text { NPV } \\
(\$ / \text { pig) }\end{array}$ & $\begin{array}{c}\text { Payback } \\
\text { (years) }\end{array}$ & $\begin{array}{c}\text { NPV } \\
\text { (S/pig) }\end{array}$ & $\begin{array}{c}\text { Payback } \\
\text { (years) }\end{array}$ \\
\hline 50 & -1191.62 & 404.4 & -1181.44 & 280 & -1161.12 & 173.3 \\
100 & -620.93 & 214.0 & -610.76 & 148.1 & -590.42 & 91.7 \\
200 & -329.18 & 114.4 & -318.80 & 79.2 & -298.04 & 49 \\
400 & -178.95 & 64.7 & -168.46 & 44.8 & -147.49 & 27.7 \\
600 & -127.24 & 47.8 & -116.71 & 33.1 & -95.67 & 20.5 \\
800 & -100.75 & 39.2 & -90.21 & 27.2 & -69.13 & 16.8 \\
1,000 & -84.53 & 34 & -73.98 & 23.5 & -52.88 & 14.6 \\
1,500 & -62.37 & 26.9 & -51.83 & 18.6 & -30.75 & 11.5 \\
2,000 & -51.06 & 23.3 & -40.57 & 16.2 & -19.60 & 10 \\
3,000 & -39.46 & 19.8 & -29.09 & 13.7 & -8.35 & 8.4 \\
5,000 & -29.71 & 16.7 & -19.49 & 11.5 & 0.97 & 7.1 \\
10,000 & -21.74 & 14.2 & -11.64 & 9.8 & 8.55 & 6 \\
15,000 & -18.78 & 13.2 & -8.74 & 9.1 & 11.34 & 5.6 \\
20,000 & -17.18 & 12.7 & -7.17 & 8.7 & 8.21 & 5.4 \\
\hline
\end{tabular}

Table 32: Finishing System Methane Recovery Economics

\begin{tabular}{|c|c|c|c|c|c|c|}
\hline \multirow{2}{*}{$\begin{array}{l}\text { Herd } \\
\text { (\#) }\end{array}$} & \multicolumn{2}{|c|}{ Scenario } & \multicolumn{2}{|c|}{ Scenario \#5 } & \multicolumn{2}{|c|}{ Scenario \#6 } \\
\hline & $\begin{array}{c}\text { NPV } \\
\text { (S/pig) }\end{array}$ & $\begin{array}{l}\text { Payback } \\
\text { (years) }\end{array}$ & $\begin{array}{c}\text { NPV } \\
\text { (S/pig) }\end{array}$ & $\begin{array}{l}\text { Payback } \\
\text { (years) }\end{array}$ & $\begin{array}{c}\text { NPV } \\
\text { (S/pig) }\end{array}$ & $\begin{array}{l}\text { Payback } \\
\text { (years) }\end{array}$ \\
\hline $\begin{array}{c}50 \\
100\end{array}$ & $\begin{array}{l}-1172.20 \\
-601.52\end{array}$ & $\begin{array}{l}218.8 \\
115.8\end{array}$ & $\begin{array}{r}-629.66 \\
-323.58\end{array}$ & $\begin{array}{c}404.4 \\
214\end{array}$ & $\begin{array}{l}-610.24 \\
-304.17\end{array}$ & $\begin{array}{l}218.8 \\
115.8\end{array}$ \\
\hline 200 & -309.77 & 62.5 & -195.66 & 114.4 & -147.51 & 62.5 \\
\hline 400 & -159.54 & 35.5 & -102.67 & 64.7 & -66.83 & 35.5 \\
\hline 600 & -107.83 & 26.3 & -70.66 & 47.8 & -39.07 & 26.6 \\
\hline 800 & -81.34 & 21.6 & -54.26 & 39.2 & -24.84 & 21.6 \\
\hline 1,000 & -68.34 & 20.2 & -44.22 & 34.0 & -16.13 & 18.7 \\
\hline 1,500 & -42.96 & 14.8 & -30.53 & 26.9 & -4.27 & 14.8 \\
\hline 2,000 & -31.65 & 12.8 & -17.69 & 23.3 & 1.72 & 12.8 \\
\hline 3,000 & -20.05 & 10.8 & -11.64 & 19.8 & 7.77 & 10.8 \\
\hline 5,000 & -10.31 & 9.0 & -6.61 & 16.7 & 12.80 & 9.0 \\
\hline 10,000 & -2.33 & 7.6 & -2.52 & 14.2 & 16.89 & 7.6 \\
\hline 15,000 & 0.62 & 7.0 & -1.01 & 13.2 & 18.40 & 7.0 \\
\hline 20,000 & 0.11 & 6.7 & -0.19 & 12.7 & 19.22 & 6.7 \\
\hline
\end{tabular}

Table 33: Dairy Tie Stall with Plug Flow Digester Energy Production and Costs

\begin{tabular}{|c|c|c|c|c|c|c|c|}
\hline $\begin{array}{l}\text { Herd } \\
\text { (\#) }\end{array}$ & $\begin{array}{c}\text { Total } \\
\text { Manure } \\
\text { (gal/d) }\end{array}$ & $\begin{array}{c}\text { Gas } \\
\text { Production } \\
\text { (cu fi/d) }\end{array}$ & $\begin{array}{l}\text { Max. } \\
\text { Energy } \\
\text { (kwh) }\end{array}$ & $\begin{array}{c}\text { Mix } \\
\text { Tank } \\
\text { (S) }\end{array}$ & $\begin{array}{c}\text { Plug Flow } \\
\text { Digestet } \\
\text { (S) }\end{array}$ & $\begin{array}{c}\text { Generator } \\
\text { Building } \\
\text { (S) }\end{array}$ & $\begin{array}{c}\text { Total } \\
\text { Cost } \\
(\$)\end{array}$ \\
\hline $\begin{array}{c}50 \\
100 \\
200 \\
400 \\
600 \\
800 \\
1,000 \\
1,500 \\
2,000 \\
3,000 \\
5,000\end{array}$ & $\begin{array}{c}899 \\
1,798 \\
3,596 \\
7,193 \\
10,766 \\
14,374 \\
17,982 \\
26,972 \\
35,963 \\
53,945 \\
89,908\end{array}$ & $\begin{array}{c}2,748 \\
5,496 \\
10,991 \\
21,982 \\
32,911 \\
43,933 \\
54,955 \\
82,379 \\
109,910 \\
164,865 \\
274,597\end{array}$ & $\begin{array}{c}71,540 \\
143,080 \\
286,525 \\
573,050 \\
857,750 \\
1,145,370 \\
1,432,625 \\
2,147,660 \\
2,863,425 \\
4,298,240 \\
7,159,110\end{array}$ & $\begin{array}{l}15,262 \\
16,152 \\
17,462 \\
19,419 \\
20,994 \\
22,391 \\
23,664 \\
26,497 \\
29,024 \\
33,552 \\
41,469\end{array}$ & $\begin{array}{c}26,140 \\
35,207 \\
50,542 \\
77,253 \\
101,590 \\
125,004 \\
147,677 \\
202,157 \\
254,816 \\
356,984 \\
554,525\end{array}$ & $\begin{array}{r}23,575 \\
32,150 \\
49,344 \\
83,688 \\
117,812 \\
152,288 \\
186,719 \\
272,600 \\
358,219 \\
530,200 \\
873,112\end{array}$ & $\begin{array}{c}89,977 \\
108,509 \\
142,348 \\
205,359 \\
265,397 \\
324,683 \\
383,060 \\
526,078 \\
667,060 \\
945,735 \\
1,494,107\end{array}$ \\
\hline
\end{tabular}


Table 34: Dairy Tie Stall with Plug Flow Digester Methane Recovery Economics

\begin{tabular}{|c|c|c|c|c|c|c|}
\hline \multirow{2}{*}{ Herd } & \multicolumn{2}{|c|}{ Scenario \#1 } & \multicolumn{2}{c|}{ Scenario \#2 } & \multicolumn{2}{c|}{ Scenario \#3 } \\
\cline { 2 - 7 }$(\#)$ & $\begin{array}{c}\text { NPV } \\
\text { (S/lac. cow) }\end{array}$ & $\begin{array}{c}\text { Payback } \\
\text { (years) }\end{array}$ & $\begin{array}{c}\text { NPV } \\
\text { (\$/lac. cow) }\end{array}$ & $\begin{array}{c}\text { Payback } \\
\text { (years) }\end{array}$ & $\begin{array}{c}\text { NPV } \\
\text { (S/lac. cow) }\end{array}$ & $\begin{array}{c}\text { Payback } \\
\text { (years) }\end{array}$ \\
\hline 50 & -1187.68 & 31.1 & -1021.60 & 21.5 & -689.46 & 13.3 \\
100 & -611.29 & 20.2 & -454.25 & 13.9 & -140.17 & 8.6 \\
200 & -385.95 & 17.9 & -258.85 & 12 & -4.65 & 7.2 \\
400 & -281.96 & 17.2 & -176.04 & 11.1 & 35.79 & 6.5 \\
600 & -242.76 & 16.6 & -143.90 & 10.5 & 53.81 & 6.1 \\
800 & -222.79 & 16.2 & -127.46 & 10.2 & 63.19 & 5.9 \\
1,000 & -209.99 & 15.9 & -116.78 & 10 & 69.63 & 5.7 \\
1,500 & -191.05 & 15.4 & -100.66 & 9.5 & 80.10 & 5.4 \\
2,000 & -180.76 & 15.1 & -91.79 & 9.3 & 86.15 & 5.3 \\
3,000 & -169.67 & 14.7 & -82.11 & 9 & 93.01 & 5.1 \\
5,000 & -159.08 & 14.3 & -72.65 & 8.7 & 100.21 & 4.9 \\
\hline
\end{tabular}

Table 35: Dairy Tie Stall with Plug Flow Digester Methane Recovery Economics

\begin{tabular}{|c|c|c|c|c|c|c|}
\hline \multirow{2}{*}{ Herd } & \multicolumn{2}{|c|}{ Scenario \#4 } & \multicolumn{2}{c|}{ Scenario \#5 } & \multicolumn{2}{c|}{ Scenario \#6 } \\
\cline { 2 - 7 }$(\#)$ & $\begin{array}{c}\text { NPV } \\
(\text { S/lac. cow) }\end{array}$ & $\begin{array}{c}\text { Payback } \\
\text { (years) }\end{array}$ & $\begin{array}{c}\text { NPV } \\
\text { (S/lac. cow) }\end{array}$ & $\begin{array}{c}\text { Payback } \\
\text { (years) }\end{array}$ & $\begin{array}{c}\text { NPV } \\
\text { (S/ac. cow) }\end{array}$ & $\begin{array}{c}\text { Payback } \\
\text { (years) }\end{array}$ \\
\hline 50 & -1129.00 & 26.8 & -482.86 & 31.1 & -424.16 & 26.8 \\
100 & -559.06 & 17.6 & -186.29 & 20.2 & -127.61 & 17.3 \\
200 & -327.27 & 14.6 & -106.87 & 17.9 & -48.19 & 14.5 \\
400 & -223.28 & 13.2 & -80.72 & 17.1 & -22.04 & 13.1 \\
600 & -184.08 & 12.5 & -69.41 & 16.6 & -10.72 & 12.4 \\
800 & -164.10 & 11.9 & -63.67 & 16.2 & -4.99 & 11.9 \\
1,000 & -151.07 & 11.6 & -59.77 & 15.9 & -1.10 & 11.6 \\
1,500 & -132.37 & 11 & -55.37 & 15.6 & 5.00 & 11 \\
2,000 & -122.08 & 10.7 & -51.83 & 15.3 & 8.56 & 10.7 \\
3,000 & -110.99 & 10.3 & -47.72 & 14.9 & 12.65 & 10.3 \\
5,000 & -100.40 & 10 & -43.70 & 14.5 & 16.64 & 10 \\
\hline
\end{tabular}

Table 36: Dairy Tie Stall with Complete Mix Digester Energy Production and Costs

\begin{tabular}{|c|c|c|c|c|c|c|c|}
\hline Herd & $\begin{array}{c}\text { Total } \\
\text { Manure } \\
\text { (gaVd) }\end{array}$ & $\begin{array}{c}\text { Production } \\
\text { (cu f f/d) }\end{array}$ & $\begin{array}{c}\text { Max. } \\
\text { Energy } \\
(\mathrm{kwwh})\end{array}$ & $\begin{array}{c}\text { Mix } \\
\text { Tank } \\
(\mathbf{S})\end{array}$ & $\begin{array}{c}\text { Plug Flow } \\
\text { Digester } \\
(\mathbf{S})\end{array}$ & $\begin{array}{c}\text { Generator } \\
\text { Building } \\
(\mathbf{S})\end{array}$ & $\begin{array}{c}\text { Total } \\
\text { Cost } \\
(\mathbf{S})\end{array}$ \\
\hline 50 & $\mathbf{8 9 9}$ & 2,748 & 71,540 & 15,262 & 20,188 & 23,575 & $\mathbf{8 4 , 0 2 5}$ \\
100 & 1,798 & 5,496 & 143,080 & 16,152 & 25,509 & 32,150 & 98,811 \\
200 & 3,596 & 10,991 & 286,525 & 17,462 & 34,465 & 49,344 & 126,270 \\
400 & 7,193 & 21,982 & 573,050 & 19,419 & 49,992 & 83,688 & 178,098 \\
600 & 10,766 & 32,911 & 857,750 & 20,994 & 64,093 & 117,812 & 227,899 \\
800 & 14,374 & 43,933 & $1,145,370$ & 22,391 & 77,632 & 152,288 & 277,310 \\
1,000 & 17,982 & 54,955 & $1,432,625$ & 23,664 & 90,724 & 186,719 & 326,107 \\
1,500 & 26,972 & 82,379 & $2,147,660$ & 26,497 & 122,133 & 272,600 & 446,230 \\
2,000 & 35,963 & 109,910 & $2,863,425$ & 29,024 & 152,446 & 358,438 & 564,907 \\
3,000 & 53,945 & 164,865 & $4,298,240$ & 33,552 & 21,172 & 530,200 & 799,924 \\
5,000 & 89,908 & 274,775 & $7,163,490$ & 41,469 & 324,532 & 873,638 & $1,264,639$ \\
\hline
\end{tabular}

Table 37: Dairy Tie Stall with Complete Mix Digester Methane Recovery Economics

\begin{tabular}{|c|c|c|c|c|c|c|}
\hline \multirow{2}{*}{$\begin{array}{l}\text { Herd } \\
\text { (A) }\end{array}$} & \multicolumn{2}{|c|}{ Scenario \#1 } & \multicolumn{2}{|c|}{ Scenario \#2 } & \multicolumn{2}{|c|}{ Scenario \#3 } \\
\hline & $\begin{array}{c}\text { NPV } \\
\text { (S/ac. cow) }\end{array}$ & $\begin{array}{c}\text { Payback } \\
\text { (years) }\end{array}$ & $\begin{array}{c}\text { NPV } \\
\text { (S/ac. cow) }\end{array}$ & $\begin{array}{c}\text { Payback } \\
\text { (years) }\end{array}$ & $\begin{array}{c}\text { NPV } \\
\text { (S/ac. cow) }\end{array}$ & $\begin{array}{c}\text { Payback } \\
\text { (years) }\end{array}$ \\
\hline $\begin{array}{c}50 \\
100 \\
200 \\
400 \\
600 \\
800 \\
1,000 \\
1,500 \\
2,000 \\
3,000 \\
5,000\end{array}$ & $\begin{array}{l}-1,087.12 \\
-529.37 \\
-318.05 \\
-224.39 \\
-189.97 \\
-172.77 \\
-161.88 \\
-146.19 \\
-137.72 \\
-128.61 \\
-120.41\end{array}$ & $\begin{array}{l}29.0 \\
18.4 \\
15.9 \\
14.9 \\
14.2 \\
13.9 \\
13.6 \\
13.3 \\
12.8 \\
12.5 \\
12.1\end{array}$ & $\begin{array}{c}-921.04 \\
-372.33 \\
-190.95 \\
-118.47 \\
-91.11 \\
-77.44 \\
-68.68 \\
-55.81 \\
48.75 \\
-41.05 \\
-33.98 \\
\end{array}$ & $\begin{array}{l}20.1 \\
12.7 \\
10.6 \\
9.6 \\
9.0 \\
8.7 \\
8.5 \\
8.1 \\
7.9 \\
7.6 \\
7.4 \\
\end{array}$ & $\begin{array}{c}-588.88 \\
-58.25 \\
63.26 \\
93.36 \\
106.60 \\
113.21 \\
117.74 \\
124.95 \\
129.19 \\
134.06 \\
138.87\end{array}$ & $\begin{array}{l}12.4 \\
7.8 \\
6.4 \\
5.6 \\
5.2 \\
5.0 \\
4.8 \\
4.6 \\
4.5 \\
4.3 \\
4.1\end{array}$ \\
\hline
\end{tabular}


Table 38: Dairy Tie Stall with Complete Mix Digester Methane Recovery Economics

\begin{tabular}{|c|c|c|c|c|c|c|}
\hline \multirow{2}{*}{ Herd } & \multicolumn{2}{|c|}{ Scenario 4 } & \multicolumn{2}{c|}{ Scenario \#5 } & \multicolumn{2}{c|}{ Scenario \#6 } \\
\cline { 2 - 7 }$(\#)$ & $\begin{array}{c}\text { NPV } \\
\text { (S/ac. cow) }\end{array}$ & $\begin{array}{c}\text { Payback } \\
\text { (years) }\end{array}$ & $\begin{array}{c}\text { NPV } \\
\text { (\$/ac. cow) }\end{array}$ & $\begin{array}{c}\text { Payback } \\
\text { (years) }\end{array}$ & $\begin{array}{c}\text { NPV } \\
\text { (S/ac. cow) }\end{array}$ & $\begin{array}{c}\text { Payback } \\
\text { (years) }\end{array}$ \\
\hline 50 & $-1,028.44$ & 25.1 & -428.92 & 29.0 & -370.24 & 25.1 \\
100 & -470.69 & 15.7 & -142.35 & 18.4 & -83.67 & 15.7 \\
200 & -259.37 & 12.9 & -70.76 & 15.9 & -11.77 & 12.9 \\
400 & -165.71 & 11.4 & -50.00 & 14.9 & 8.84 & 11.4 \\
600 & -131.28 & 10.6 & -41.20 & 14.2 & 17.59 & 10.6 \\
800 & -114.08 & 10.2 & -37.00 & 13.9 & 21.84 & 10.2 \\
1,000 & -103.20 & 9.8 & -34.16 & 13.6 & 24.71 & 9.8 \\
1,500 & -87.51 & 9.4 & -29.68 & 13.1 & 29.17 & 9.3 \\
2,000 & -79.04 & 9.1 & -27.09 & 12.8 & 31.75 & 9.1 \\
3,000 & -69.93 & 8.7 & -24.18 & 12.5 & 34.67 & 8.7 \\
5,000 & -61.73 & 8.4 & -21.35 & 12.1 & 37.48 & 8.4 \\
\hline
\end{tabular}

Table 39: Dairy Free Stall with Plug Flow Digester Energy Production and Costs

\begin{tabular}{|c|c|c|c|c|c|c|c|}
\hline $\begin{array}{l}\text { Herd } \\
\text { (\#) }\end{array}$ & $\begin{array}{c}\text { Total } \\
\text { Manure } \\
\text { (gal/d) }\end{array}$ & $\begin{array}{c}\text { Gas } \\
\text { Production } \\
\text { (cu fUd) }\end{array}$ & $\begin{array}{l}\text { Max. } \\
\text { Energy } \\
(\mathbf{k w b})\end{array}$ & $\begin{array}{c}\text { Mix } \\
\text { Tank } \\
(\boldsymbol{S})\end{array}$ & $\begin{array}{l}\text { Plug Flow } \\
\text { Digester } \\
\text { (S) }\end{array}$ & $\begin{array}{c}\text { Generator } \\
\text { Building } \\
\text { (S) }\end{array}$ & $\begin{array}{l}\text { Total } \\
\text { Cost } \\
\text { (S) }\end{array}$ \\
\hline 50 & 905 & 2,763 & 71,905 & 15,269 & 26,203 & 23,619 & 90,091 \\
\hline 100 & 1,809 & 5,527 & 143,810 & 16,161 & 35,312 & 32,238 & 108,711 \\
\hline 200 & 3,619 & 11,053 & 287,985 & 17,476 & 50,722 & 49,519 & 142,717 \\
\hline 400 & 7,238 & 22,106 & 576,335 & 19,441 & 77,571 & 84,081 & 206,093 \\
\hline 600 & 10,823 & 33,066 & 861,765 & 21,017 & 101,965 & 118,294 & 266,276 \\
\hline 800 & 14,459 & 44,166 & $1,151,210$ & 22,422 & 125,545 & 152,988 & 325,955 \\
\hline 1,000 & 18,095 & 55,266 & $1,440,655$ & 23,702 & 148,378 & 187,681 & 384,762 \\
\hline 1,500 & 27,142 & 82,899 & $2,161,165$ & 26,547 & 203,164 & 274,044 & 528,755 \\
\hline 2,000 & 36,189 & 110,532 & $2,881,675$ & 29,085 & 256,124 & 360,406 & 670,616 \\
\hline 3,000 & 54,284 & 165,798 & $4,322,330$ & 33,632 & 358,882 & 533,088 & 950,602 \\
\hline 5,000 & 90,473 & 276,331 & $7,204,005$ & 41,586 & 557,585 & 878,494 & 1,502664 \\
\hline
\end{tabular}

Table 40: Dairy Free Stall with Plug Flow Digester Methane Recovery Economics

\begin{tabular}{|c|c|c|c|c|c|c|}
\hline \multirow{2}{*}{ Herd } & \multicolumn{2}{|c|}{ Scenario \#1 } & \multicolumn{2}{c|}{ Scenario \#2 } & \multicolumn{2}{c|}{ Scenario \$3 } \\
\cline { 2 - 7 }$(\#)$ & $\begin{array}{c}\text { NPV } \\
\text { (S/lac. cow) }\end{array}$ & $\begin{array}{c}\text { Payback } \\
\text { (years) }\end{array}$ & $\begin{array}{c}\text { NPV } \\
\text { (\$/lac. cow) }\end{array}$ & $\begin{array}{c}\text { Payback } \\
\text { (years) }\end{array}$ & $\begin{array}{c}\text { NPV } \\
\text { (\$/ac. cow) }\end{array}$ & $\begin{array}{c}\text { Payback } \\
\text { (years) }\end{array}$ \\
\hline 50 & $-1,187.90$ & 30.9 & $-1,020.98$ & 21.4 & -687.14 & 13.3 \\
100 & -612.47 & 20.2 & -454.97 & 13.9 & -139.97 & 8.6 \\
200 & -388.36 & 18 & -261.26 & 12 & -7.06 & 7.2 \\
400 & -284.46 & 17.3 & -178.54 & 11.1 & 33.29 & 6.5 \\
600 & -244.77 & 16.7 & -145.91 & 10.6 & 51.80 & 6.1 \\
800 & -224.97 & 16.3 & -129.65 & 10.3 & 61.00 & 5.9 \\
1,000 & -212.36 & 16.1 & -119.15 & 10 & 67.26 & 5.7 \\
1,500 & -193.60 & 15.6 & -103.22 & 9.6 & 77.55 & 5.5 \\
2,000 & -183.32 & 15.3 & -94.35 & 9.4 & 83.60 & 5.3 \\
3,000 & -171.97 & 14.9 & -84.41 & 9.1 & 90.71 & 5.1 \\
5,000 & -161.56 & 14.5 & -75.14 & 8.8 & 97.72 & 4.9 \\
\hline
\end{tabular}

Table 41: Dairy Free Stall with Plug Flow Digester Methane Recovery Economics

\begin{tabular}{|c|c|c|c|c|c|c|}
\hline \multirow{2}{*}{ Herd } & \multicolumn{2}{|c|}{ Scenario 4} & \multicolumn{2}{c|}{ Scenario \#5 } & \multicolumn{2}{c|}{ Scenario \#6 } \\
\cline { 2 - 8 } (\#) & $\begin{array}{c}\text { NPV } \\
\text { (S/ac. cow) }\end{array}$ & $\begin{array}{c}\text { Payback } \\
\text { (years) }\end{array}$ & $\begin{array}{c}\text { NPV } \\
\text { (S/lac. cow) }\end{array}$ & $\begin{array}{c}\text { Payback } \\
\text { (years) }\end{array}$ & $\begin{array}{c}\text { NPV } \\
\text { (S/lac. cow) }\end{array}$ & $\begin{array}{c}\text { Payback } \\
\text { (years) }\end{array}$ \\
\hline 50 & $-1,129.22$ & 26.8 & -482.18 & 30.9 & -423.50 & 26.8 \\
100 & -553.79 & 17.3 & -186.68 & 20.2 & -127.99 & 17.3 \\
200 & -329.68 & 14.6 & -108.86 & 18 & -50.18 & 14.6 \\
400 & -225.78 & 13.2 & -82.50 & 17.3 & -23.82 & 13.2 \\
600 & -186.09 & 12.4 & -70.84 & 16.7 & -12.16 & 12.4 \\
800 & -166.29 & 12 & -65.23 & 16.3 & -6.55 & 12 \\
1,000 & -153.68 & 11.7 & -61.53 & 16.1 & -2.85 & 11.6 \\
1,500 & -134.92 & 11.1 & -55.37 & 15.6 & 3.32 & 11.1 \\
2,000 & -124.64 & 10.8 & -51.83 & 15.3 & 6.85 & 10.8 \\
3,000 & -113.29 & 10.4 & -47.72 & 14.9 & 10.97 & 10.4 \\
5,000 & -102.88 & 10.1 & -43.70 & 14.5 & 14.98 & 10.1 \\
\hline
\end{tabular}


Table 42: Dairy Free Stall Complete Mix Digester Energy Production and Costs

\begin{tabular}{|cccccccc|}
\hline Herd & $\begin{array}{c}\text { Total } \\
\text { Manure } \\
\text { (gal/d) }\end{array}$ & $\begin{array}{c}\text { Gas } \\
\text { Production } \\
\text { (cu ftd) }\end{array}$ & $\begin{array}{c}\text { Max. } \\
\text { Energy } \\
(\mathrm{kwh})\end{array}$ & $\begin{array}{c}\text { Mix } \\
\text { Tank } \\
(\mathbf{S})\end{array}$ & $\begin{array}{c}\text { Plug Flow } \\
\text { Digester } \\
(\mathbf{S})\end{array}$ & $\begin{array}{c}\text { Generator } \\
\text { Building } \\
(\mathbf{S})\end{array}$ & $\begin{array}{c}\text { Total } \\
\text { Cost } \\
(\mathbf{S})\end{array}$ \\
\hline 50 & 905 & 2,763 & 71,905 & 15,269 & 20,225 & 23,619 & 84,112 \\
100 & 1,809 & 5,527 & 143,810 & 16,161 & 25,570 & 32,238 & 98,969 \\
200 & 3,619 & 11,053 & 287,985 & 17,476 & 34,570 & 49,519 & 126,565 \\
400 & 7,238 & 22,106 & 576,335 & 19,441 & 50,176 & 84,081 & 178,698 \\
600 & 10,823 & 33,066 & 861,765 & 21,017 & 64,309 & 118,294 & 228,620 \\
800 & 14,459 & 44,166 & $1,151,210$ & 22,422 & 77,944 & 152,988 & 278,354 \\
1,000 & 18,095 & 55,266 & $1,440,655$ & 23,702 & 91,129 & 187,681 & 327,512 \\
1,500 & 27,142 & 82,899 & $2,161,165$ & 26,547 & 122,713 & 274,044 & 448,304 \\
2,000 & 36,189 & 110,532 & $2,881,675$ & 29,085 & 153,198 & 360,406 & 567,689 \\
3,000 & 54,284 & 165,798 & $4,322,330$ & 33,632 & 212,262 & 533,088 & 803,982 \\
5,000 & 90,473 & 276,331 & $7,204,005$ & 41,586 & 326,286 & 878,494 & $1,271,366$ \\
\hline
\end{tabular}

Table 43: Dairy Free Stall Complete Mix Digester Methane Recovery Economics

\begin{tabular}{|c|c|c|c|c|c|c|}
\hline \multirow{2}{*}{$\begin{array}{c}\text { Herd } \\
\text { (\#) }\end{array}$} & \multicolumn{2}{|c|}{ Scenario \#1 } & \multicolumn{2}{c|}{ Scenario \#2 } & \multicolumn{2}{c|}{ Scenario \#3 } \\
\cline { 2 - 7 } & $\begin{array}{c}\text { NPV } \\
\text { (S/lac. cow) }\end{array}$ & $\begin{array}{c}\text { Payback } \\
\text { (years) }\end{array}$ & $\begin{array}{c}\text { NPV } \\
\text { (S/ac. cow) }\end{array}$ & $\begin{array}{c}\text { Payback } \\
\text { (years) }\end{array}$ & $\begin{array}{c}\text { NPV } \\
\text { (\$/lac. cow) }\end{array}$ & $\begin{array}{c}\text { Payback } \\
\text { (years) }\end{array}$ \\
\hline 50 & $-1,086.90$ & 28.9 & -919.98 & 20 & -586.12 & 12.4 \\
100 & -530.18 & 18.4 & -372.68 & 12.6 & -57.56 & 7.8 \\
200 & -320.14 & 15.9 & -193.04 & 10.7 & 61.17 & 6.4 \\
400 & -226.61 & 15 & -120.69 & 9.7 & 91.14 & 5.6 \\
600 & -191.76 & 14.3 & -92.90 & 9.1 & 104.81 & 5.2 \\
800 & -174.71 & 14.0 & -79.39 & 8.8 & 111.27 & 5.0 \\
1,000 & -164.00 & 13.7 & -70.79 & 8.5 & 115.62 & 4.9 \\
1,500 & -148.29 & 13.2 & -57.91 & 8.2 & 122.86 & 4.6 \\
2,000 & -139.85 & 12.9 & -50.88 & 7.9 & 127.26 & 4.5 \\
3,000 & -130.69 & 12.6 & -43.13 & 7.7 & 131.99 & 4.3 \\
5,000 & -122.49 & 12.2 & -36.06 & 7.4 & 136.80 & 4.2 \\
\hline
\end{tabular}

Table 44: Dairy Free Stall Complete Mix Digester Methane Recovery Economics

\begin{tabular}{|c|c|c|c|c|c|c|}
\hline \multirow{2}{*}{ Herd } & \multicolumn{2}{|c|}{ Scenario \#4 } & \multicolumn{2}{c|}{ Scenario \#5 } & \multicolumn{2}{c|}{ Scenario \#6 } \\
\cline { 2 - 8 } & $\begin{array}{c}\text { NPV } \\
\text { (S/lac. cow) }\end{array}$ & $\begin{array}{c}\text { Payback } \\
\text { (years) }\end{array}$ & $\begin{array}{c}\text { NPV } \\
\text { (S/lac.cow) }\end{array}$ & $\begin{array}{c}\text { Payback } \\
\text { (years) }\end{array}$ & $\begin{array}{c}\text { NPV } \\
\text { (S/lac. cow) }\end{array}$ & $\begin{array}{c}\text { Payback } \\
\text { (years) }\end{array}$ \\
\hline 50 & $-1,028.22$ & 25 & -428.02 & 28.9 & -369.34 & 25 \\
100 & -471.50 & 15.7 & -142.54 & 18.4 & -83.86 & 15.7 \\
200 & -261.46 & 13 & -72.28 & 15.9 & -13.59 & 13 \\
400 & -167.93 & 11.5 & -51.48 & 15 & 7.21 & 11.5 \\
600 & -133.08 & 10.7 & -42.41 & 14.3 & 16.27 & 10.7 \\
800 & -116.03 & 10.2 & -38.28 & 14 & 20.41 & 10.2 \\
1,000 & -105.32 & 9.9 & -35.60 & 13.7 & 23.08 & 9.9 \\
1,500 & -89.61 & 9.4 & -31.07 & 13.2 & 27.61 & 9.4 \\
2,000 & -80.97 & 9.1 & -28.52 & 12.9 & 30.17 & 9.1 \\
3,000 & -72.00 & 8.8 & -25.57 & 12.6 & 33.11 & 8.8 \\
5,000 & -63.62 & 8.5 & -22.75 & 12.2 & 35.94 & 8.5 \\
\hline
\end{tabular}

Table 45: Economic Breakeven Points for Methane Recovery Facilities

\begin{tabular}{|c|c|c|c|c|c|c|}
\hline \multirow[t]{2}{*}{ Facility } & \multicolumn{6}{|c|}{ Head Size Breakeven Point for Each Scenario } \\
\hline & \#1 & $\# 2$ & $\# 3$ & $\# 4$ & $\# 5$ & \#6 \\
\hline Farrow to Finish ${ }^{2}$ & $>20,000$ & $>20,000$ & $>20,000$ & $>20,000$ & $>20,000$ & $>20,000$ \\
\hline Finishing & $>20,000$ & $>20,000$ & 4,792 & 13,949 & $>20,000$ & 1,856 \\
\hline Tie Stall-Plug Flow & $>5,000$ & $>5,000$ & 222 & $>5,000$ & $>5,000$ & 1,090 \\
\hline Tie Stall -Complete Mix & $>5,000$ & $>5,000$ & 148 & $>5,000$ & $>5,000$ & 314 \\
\hline Free Stall -Plug Flow & $>5,000$ & $>5,000$ & 234 & $>5,000$ & $>5,000$ & 1.230 \\
\hline Free Stall - Complete Mix & $>5,000$ & $>5,000$ & 148 & $>5,000$ & $>5,000$ & 330 \\
\hline
\end{tabular}


Table 46: Importance Rankings of Methane Meeting Participant and Producer Opinions of the Livestock Methane Recovery

\begin{tabular}{|l|c|}
\hline Barrier, Issue, or Opportunity & $\begin{array}{c}\text { Meeting Participant } \\
\text { Average Rank }\end{array}$ \\
\hline $\begin{array}{l}\text { Low rate of economic retum on methane recovery facilities } \\
\text { Interconnection difficulties with utilities/low rate for sale of excess }\end{array}$ & 2.6 \\
electricity & 3.5 \\
Few turnkey systems developed/lack of contractor knowledge in & \\
construction of methane recovery facilities & 3.6 \\
Further development of technology for on-farm methane recovery & 4.6 \\
Lack of technical information and assistance & 4.7 \\
Difficulties obtaining financing for a methane recovery system & 4.9 \\
Public awareness of the generation/utilization of methane from livestock & 5.9 \\
facilities & 6.2 \\
\hline $\begin{array}{l}\text { Lack of cooperation between various organizations (government, nonprofit, } \\
\text { etc) to promote and educate on the use of on-farm methane recovery }\end{array}$ & \\
\hline The averof of all of the responses in the category between 1 (grestest barier) to 8 (least important barier). & \\
\hline
\end{tabular}

Table 47: Policy Scenarios for the Fossil Fuel Displacement Situations

\begin{tabular}{|c|c|}
\hline Scenario & \multicolumn{1}{c|}{ Policy Scenario Description } \\
\hline 1 & $\begin{array}{l}\text { Utilize } 100 \% \text { of lowa's manure resources with no losses and no regard to economic feasibility from Appendix A. This } \\
\text { is done to give the reader an idea of the energy that resides in the manure resource. }\end{array}$ \\
\hline 2 & $\begin{array}{l}\text { Utilize } 100 \% \text { of the dairy and swine manure resources in Iowa from data in Appendix A. Dairy and swine manure, } \\
\text { due to management and physical characteristics, has the highest potential for use with methane recovery technologies. }\end{array}$ \\
\hline 3 & $\begin{array}{l}\text { Utilize } 50 \% \text { of the dairy and swine manure resources in lowa from data in Appendix A. Dairy and swine manure, due } \\
\text { to management and physical characteristics, has the highest potential for use with methane recovery technologies. }\end{array}$ \\
\hline 4 & $\begin{array}{l}\text { Utilize } 25 \% \text { of the dairy and swine manure resources in Iowa from data in Appendix A. Dairy and swine manure, due } \\
\text { to management and physical characteristies, bas the highest potential for use with methane recovery technologies. }\end{array}$ \\
\hline 5 & $\begin{array}{l}\text { Energy recovery from seenario \#3 where the electricity rate doubles from the present rate of } 6 \text { cents per kwt to } 12 \\
\text { cents per kwh for swine and dairy manure at } 100 \% \text { utilization. }\end{array}$ \\
\hline 6 & $\begin{array}{l}\text { Energy recovery from scenario \#6 where economic incentives, such as low interest loans, are used to stimulate the } \\
\text { development and implementation of methane technologies for dairy and swine manure at } 100 \% \text { utilization. }\end{array}$ \\
\hline
\end{tabular}

Table 48: Quantities of Fossil Fuels Displaced by Livestock Methane Recovery

\begin{tabular}{|c|c|c|c|c|}
\hline \multirow{2}{*}{$\begin{array}{l}\text { Policy } \\
\text { Scenario }\end{array}$} & \multicolumn{4}{|c|}{ Quantity of Fossil Fuel Needed if Used to Completely Replace the Electricity Generated from Methane } \\
\hline & $\begin{array}{c}\text { Electricity Equivalent } \\
\text { (MWH) }\end{array}$ & $\begin{array}{c}\text { Coal } \\
\text { (short tons) }\end{array}$ & $\begin{array}{l}\text { \#2 Fuel Oil } \\
\text { (barrels) }\end{array}$ & $\begin{array}{l}\text { Natural Gas/LNG } \\
\text { (million cubic ft) }\end{array}$ \\
\hline $\begin{array}{l}1 \\
2 \\
3 \\
4 \\
5 \\
6\end{array}$ & $\begin{array}{l}2,820,108.8 \\
1,384,532.1 \\
692,266.0 \\
346,133.0 \\
153,423.8 \\
230,932.8\end{array}$ & $\begin{array}{c}1,394,932.1 \\
684,841.7 \\
342,420.9 \\
171,210.4 \\
75,889.2 \\
114,228.0\end{array}$ & $\begin{array}{c}5,472,001.3 \\
2,686,478.4 \\
1,446,803.0 \\
671,619.6 \\
297,696.0 \\
448,090.6\end{array}$ & $\begin{array}{c}30,998.5 \\
15,218.7 \\
7,609.4 \\
3,804.7 \\
1,686.4 \\
2,538.4\end{array}$ \\
\hline
\end{tabular}

'Conversions from Perry's Chemical Engineering Handbook, 1984

${ }^{2}$ To calculate electricity equivalent the methane production from each scenario is converted to heat and then electricity assuming a power plant overall thermal efficiency of $30 \%$. 
Appendix B 


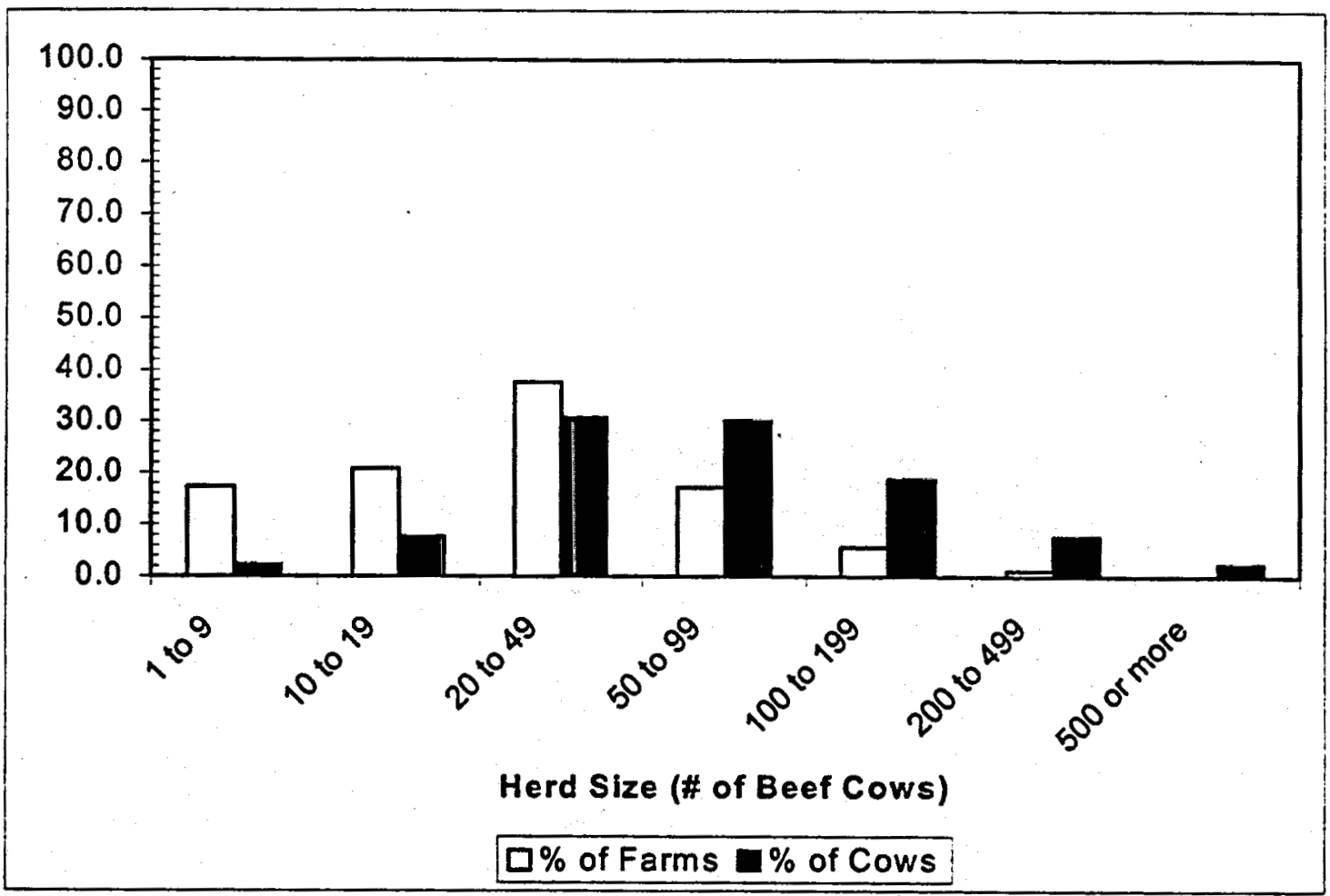

Figure 1: Farm and Inventory Based on USDA Beef Herd Classifications

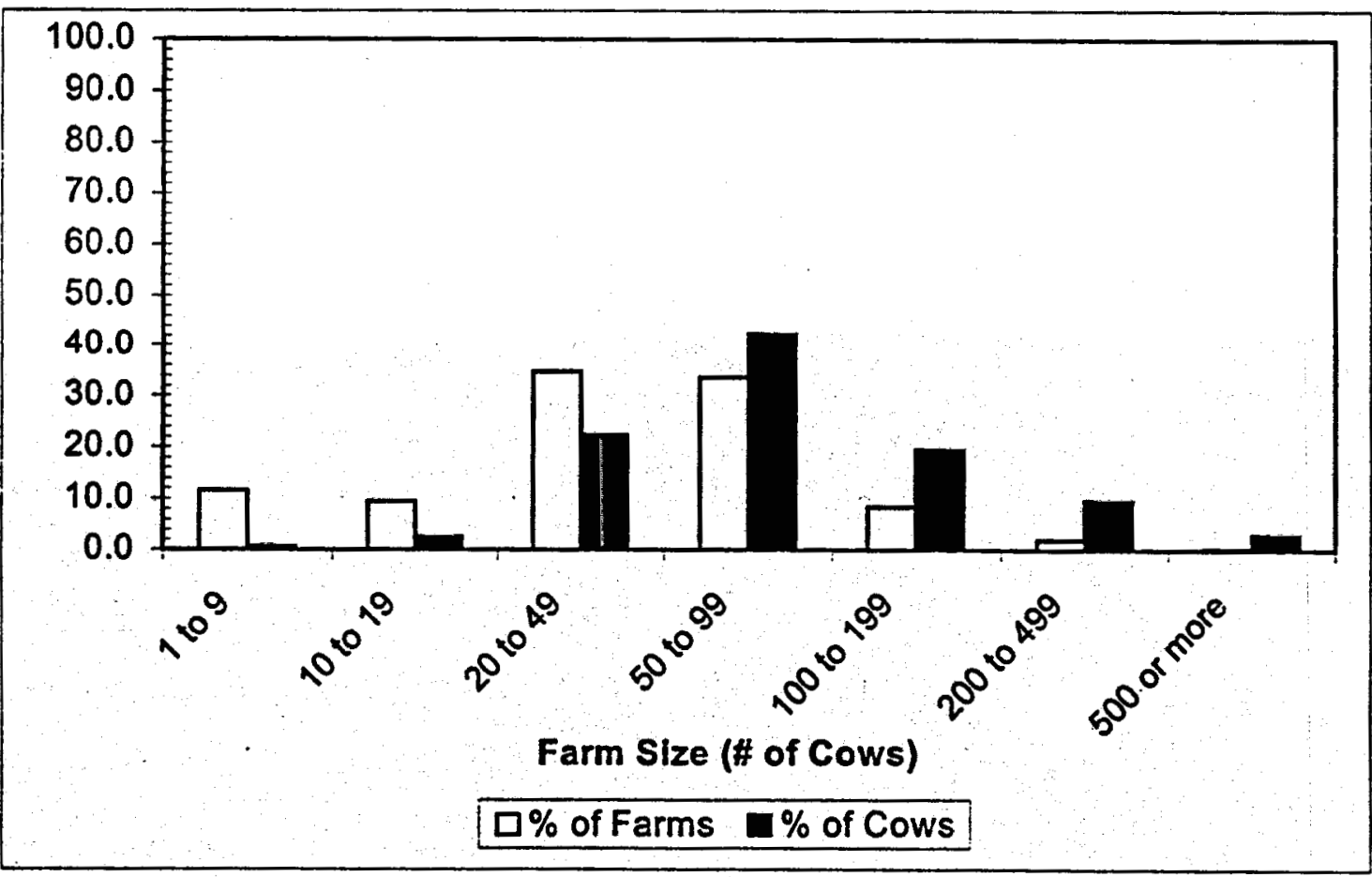

Figure 2: Farm and Inventory Based on USDA Dairy Herd Classifications 


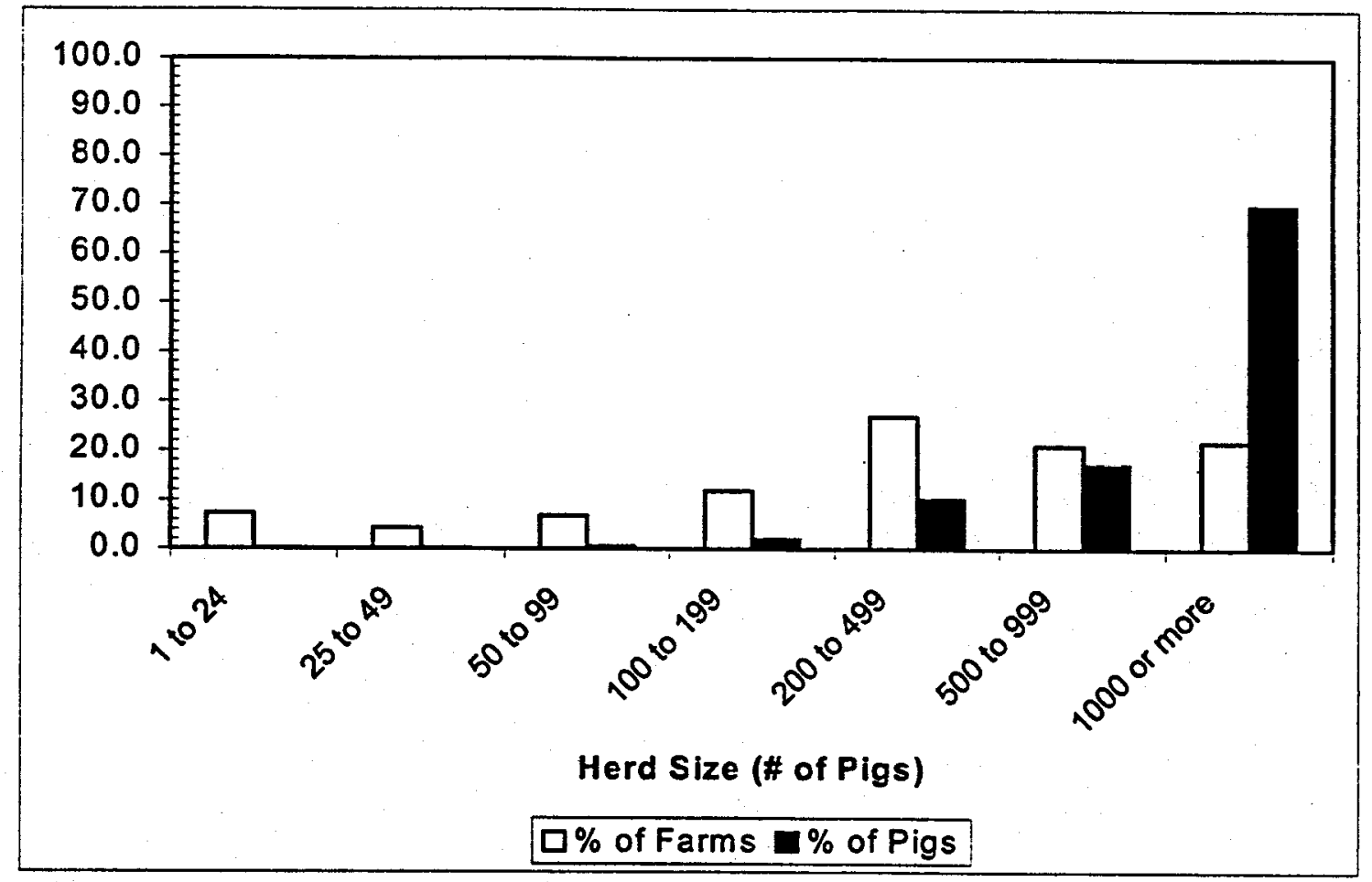

Figure 3: Swine Herd Profiles for Iowa

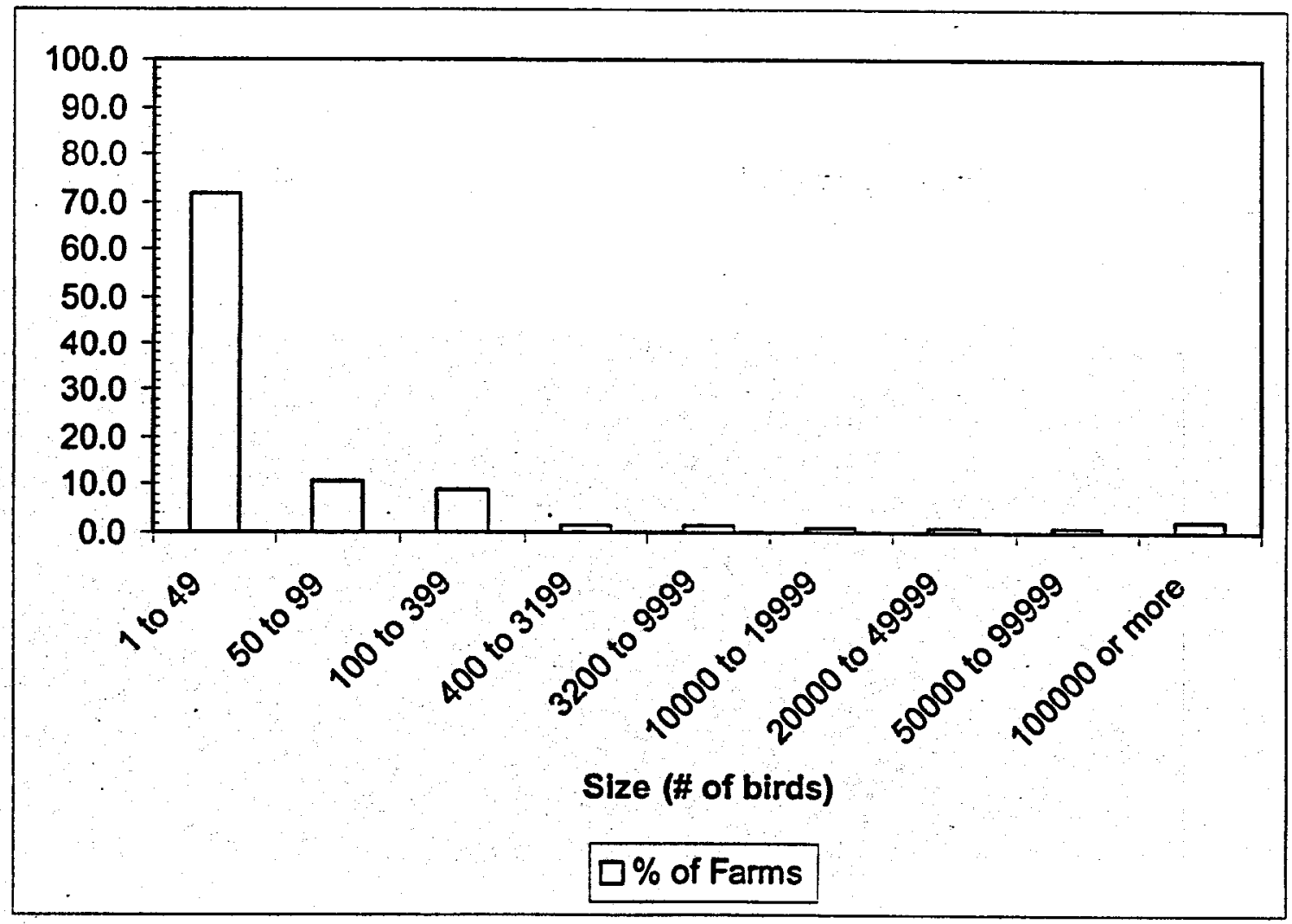

Figure 4: Profile for Poultry Layer Farm Sizes 
Farrow to Finish Operation

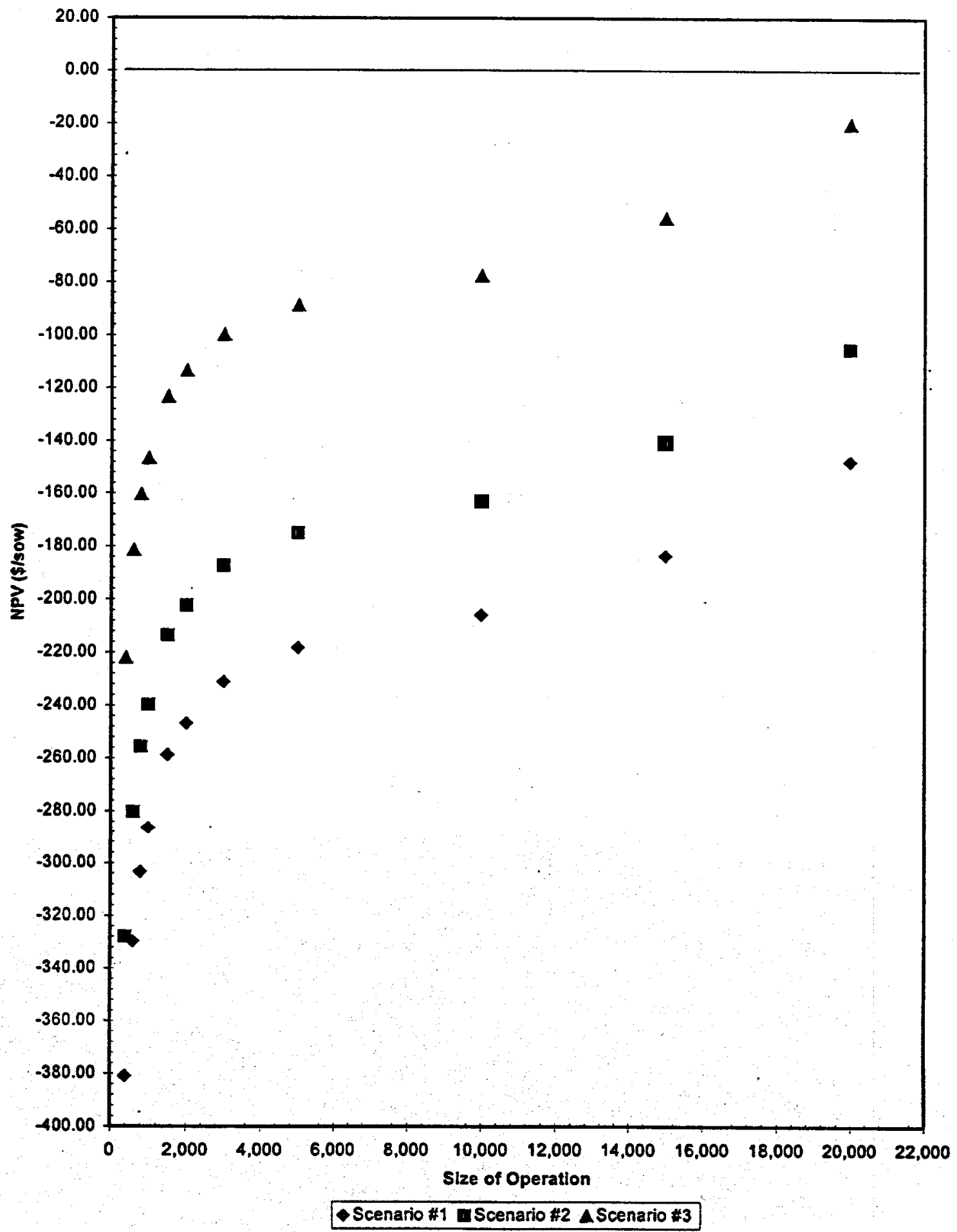

Figure 5: Net Present Value of Swine.Farrow to Finish Operation Digester 
Farrow to Finish Operation

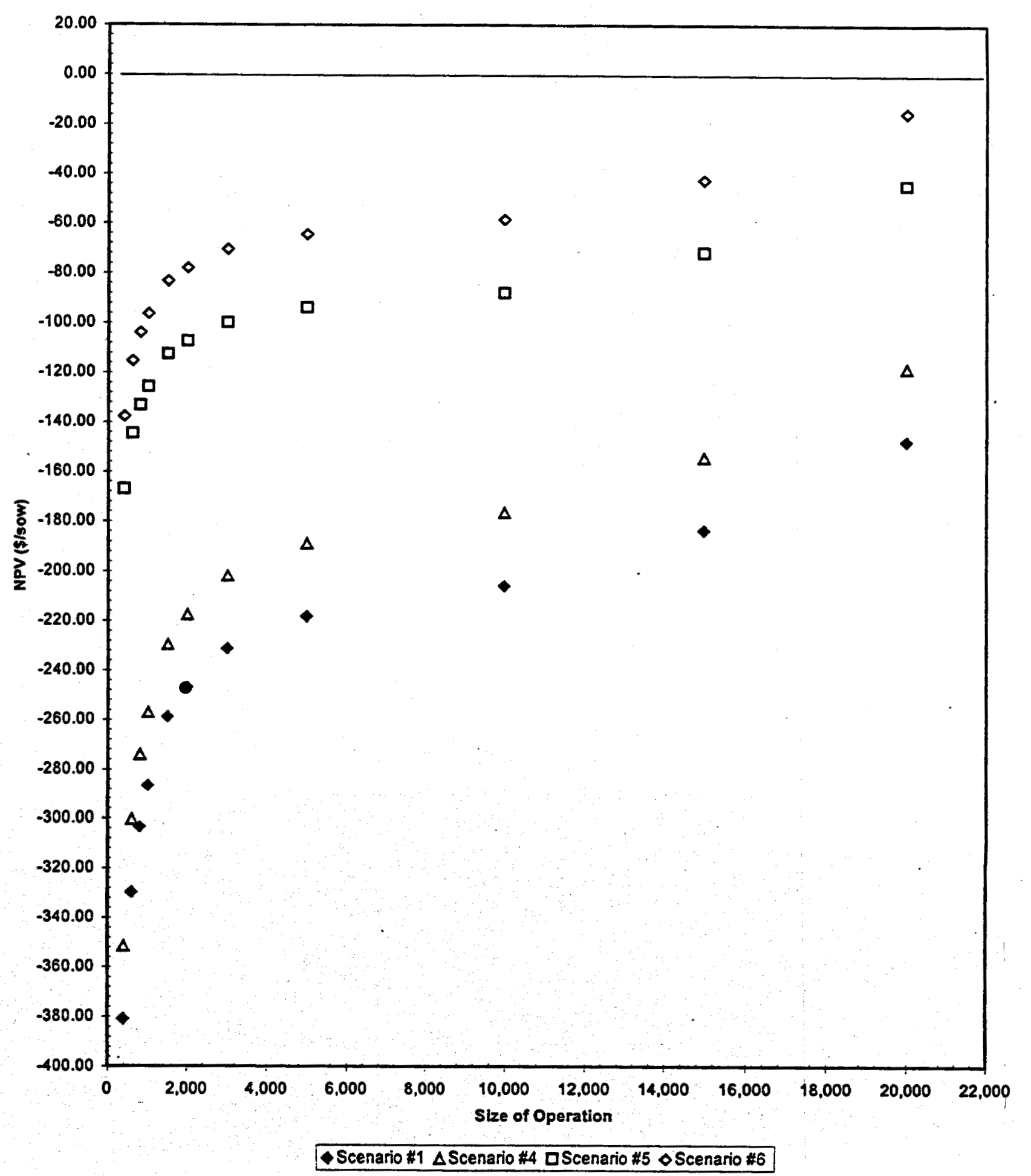

Figure 6: Net Present Value of Swine Farrow to Finish Operation Digester 
Swine Finishing Operations

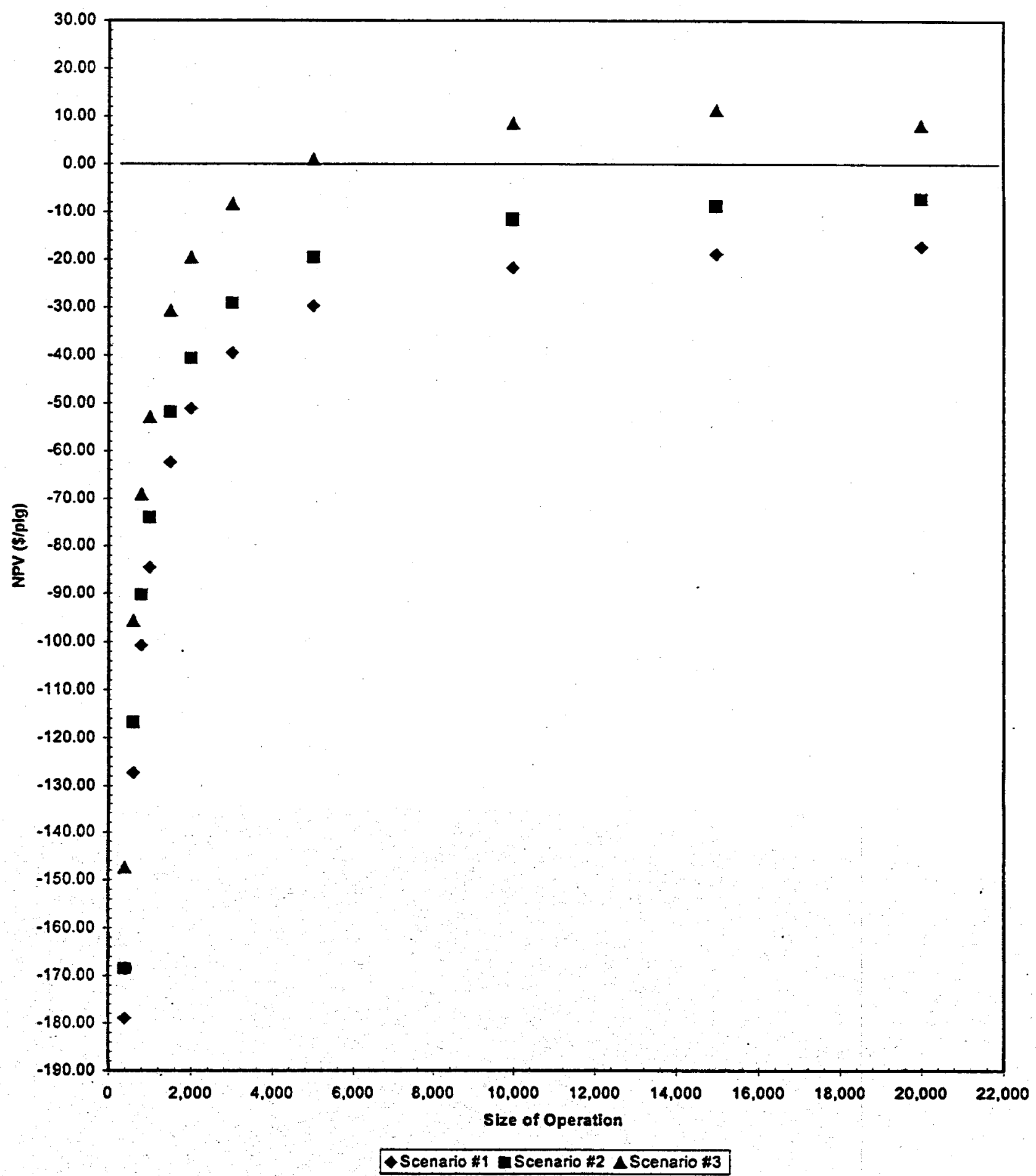

Figure 7: Net Present Value of Finishing Operation Digester 
Swine Finishing Operations

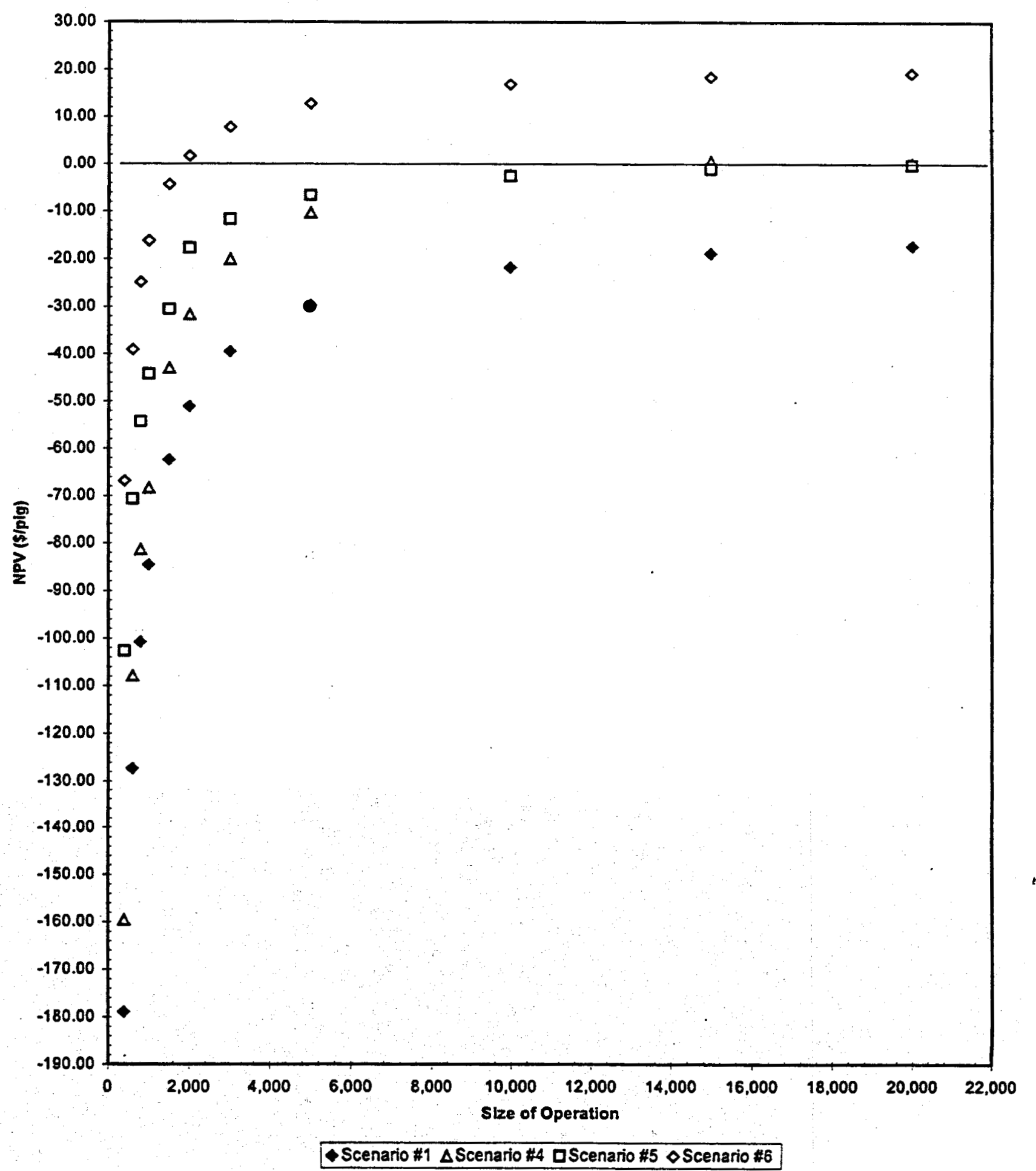

Figure 8: Net Present Value for Finishing Operation Digester 
Farrow to Finish \& Finisher Manure Production

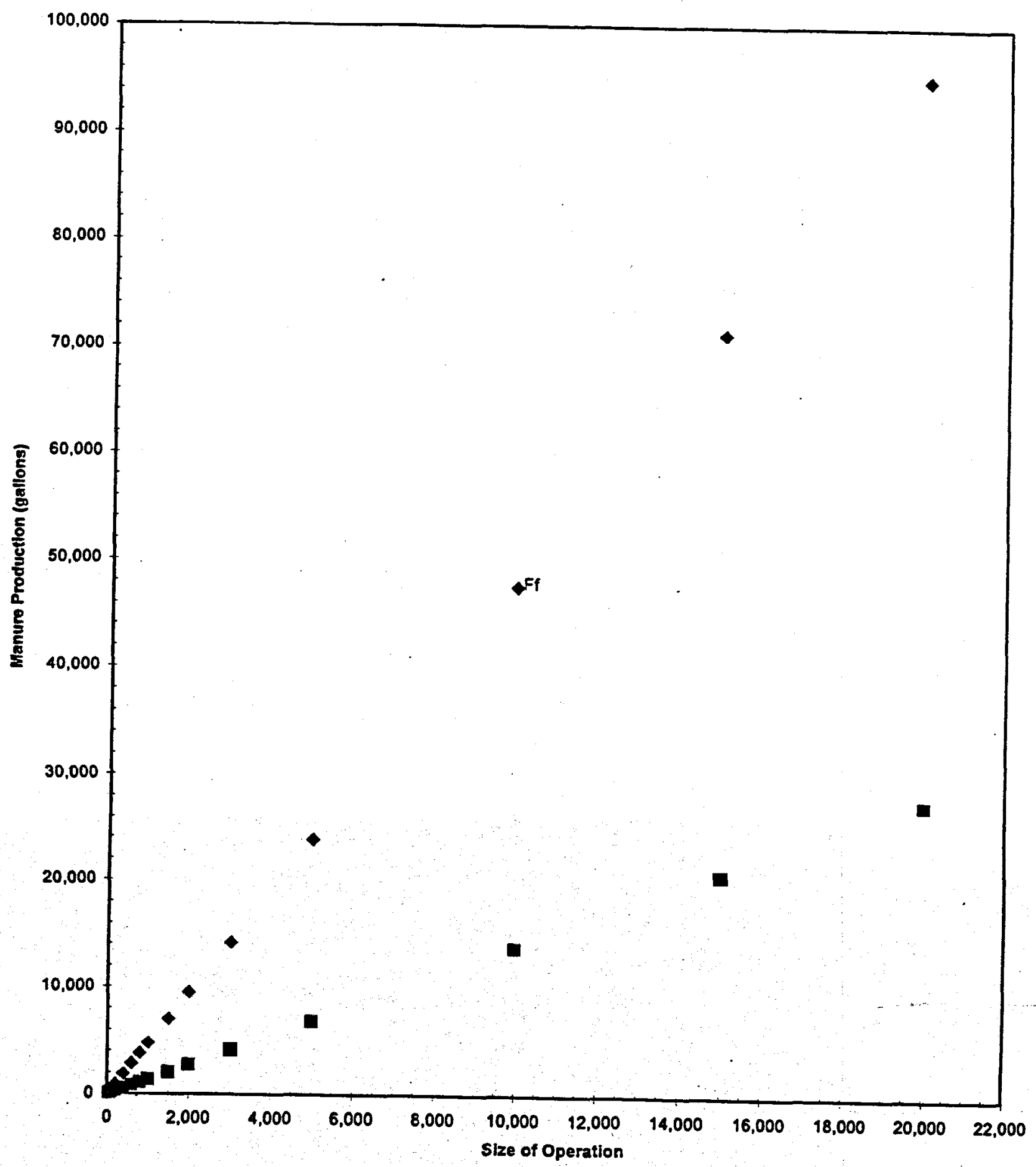

- Farrow to Finishing (\# of sows) E Finishing (\# of pigs)

Figure 9: Farrow to Finish and Finisher System Manure Production 


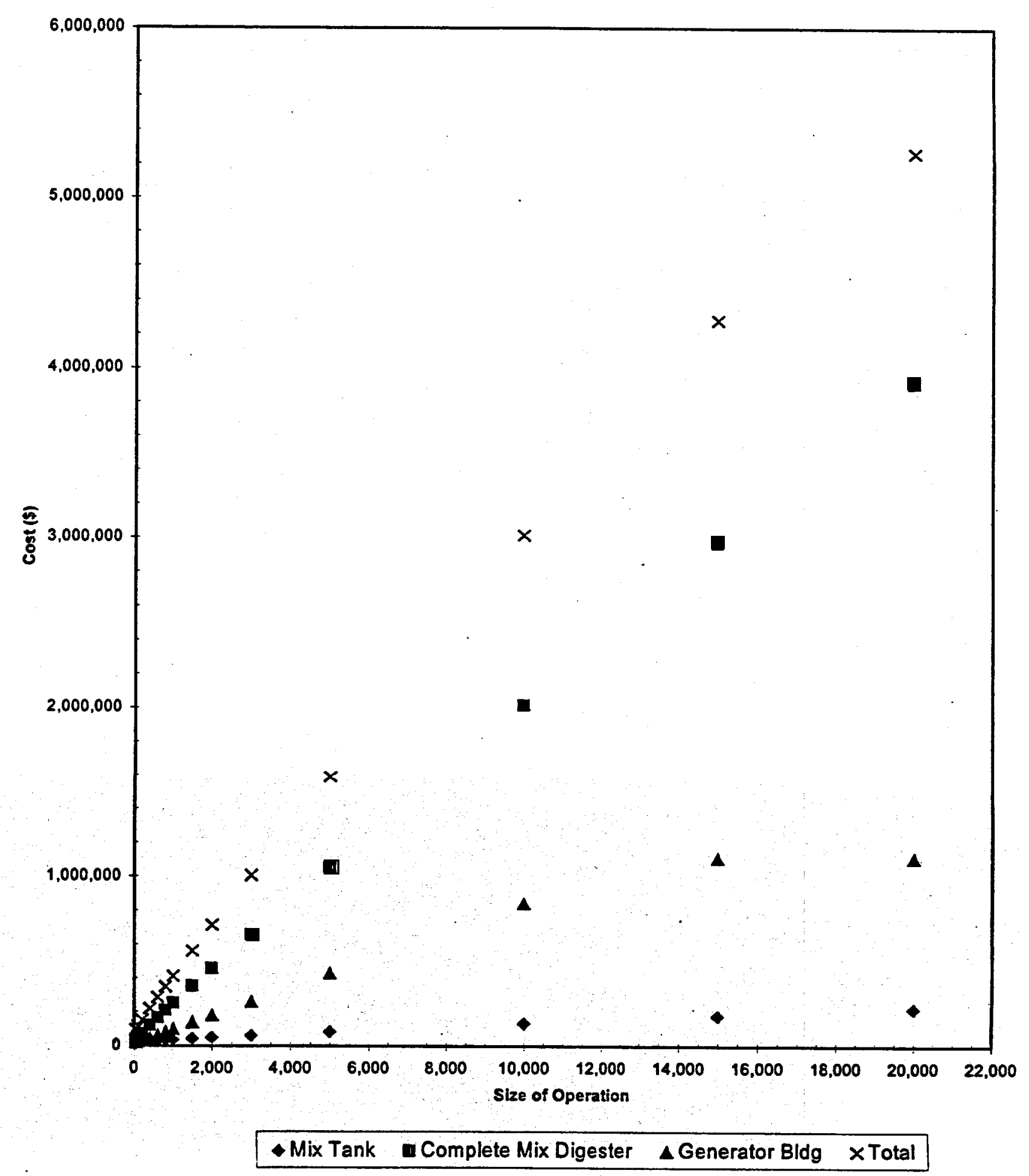

Figure 10: Farrow to Finish Operation Component and Total Costs 
Swine Finishing System Costs

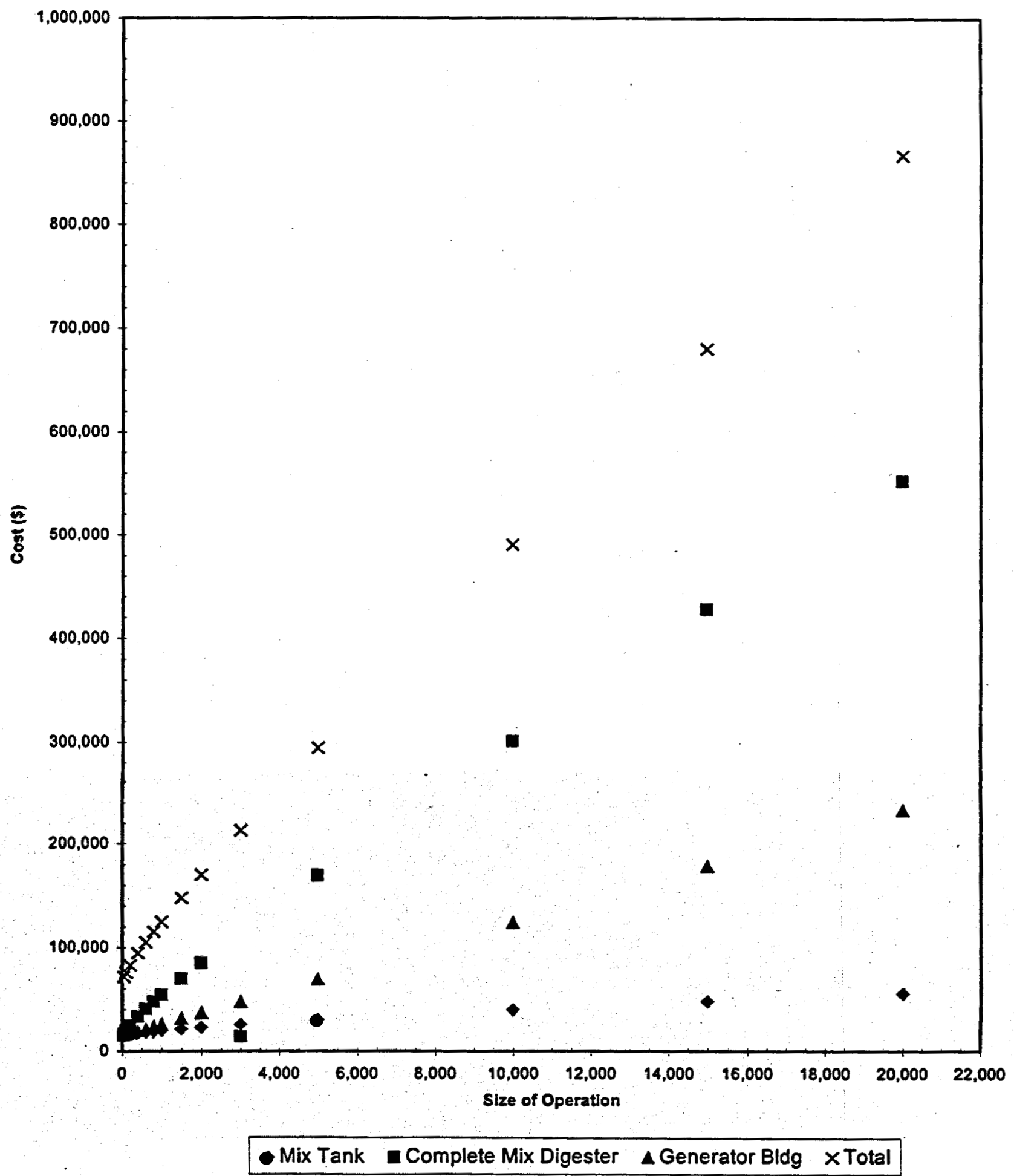

Figure 11: Finishing Operation Component and Total Costs 
Swine System Gas Production

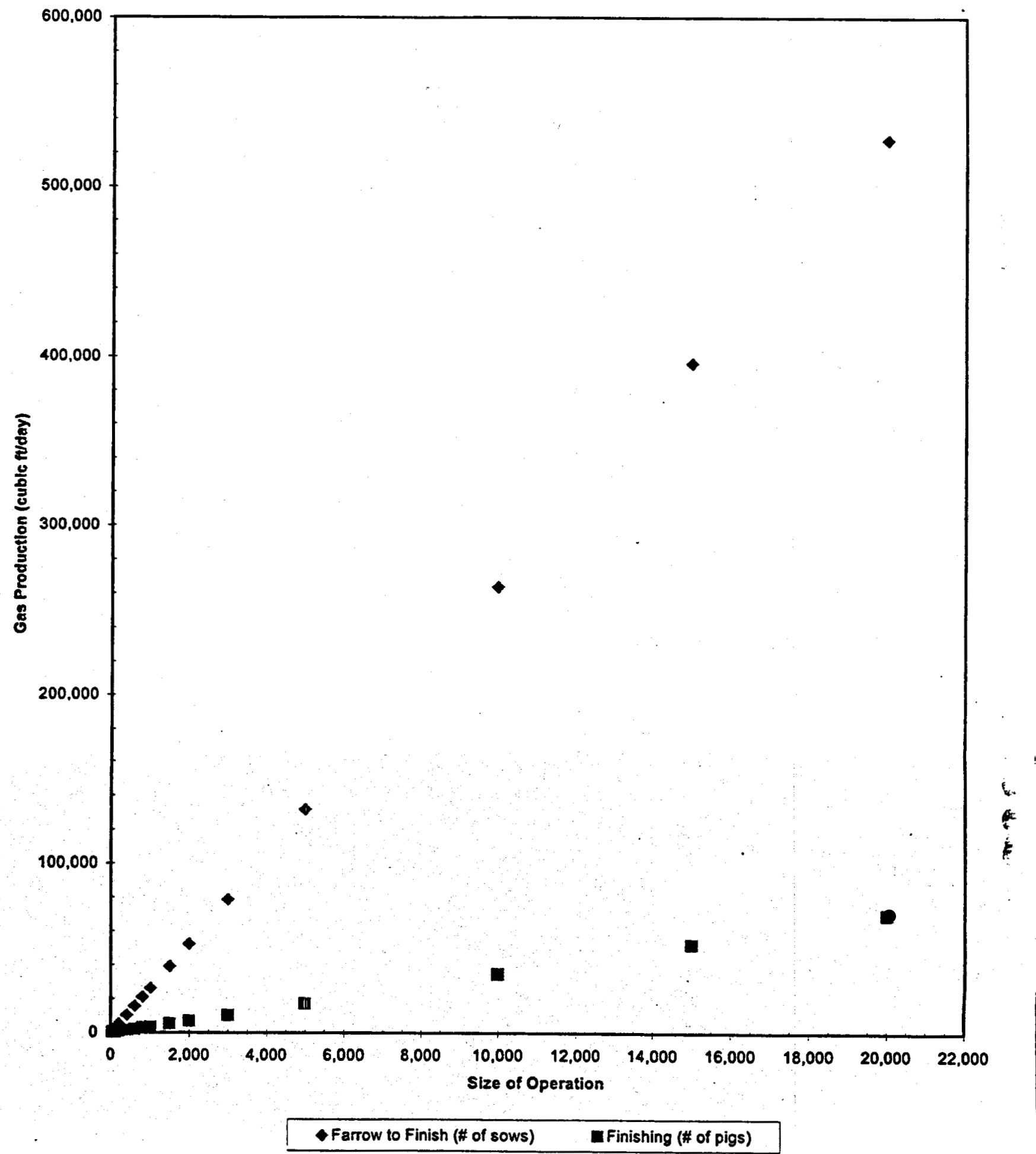

Figure 12: Farrow to Finish and Finishing System Gas Production 
Swine System Maximum Energy Production

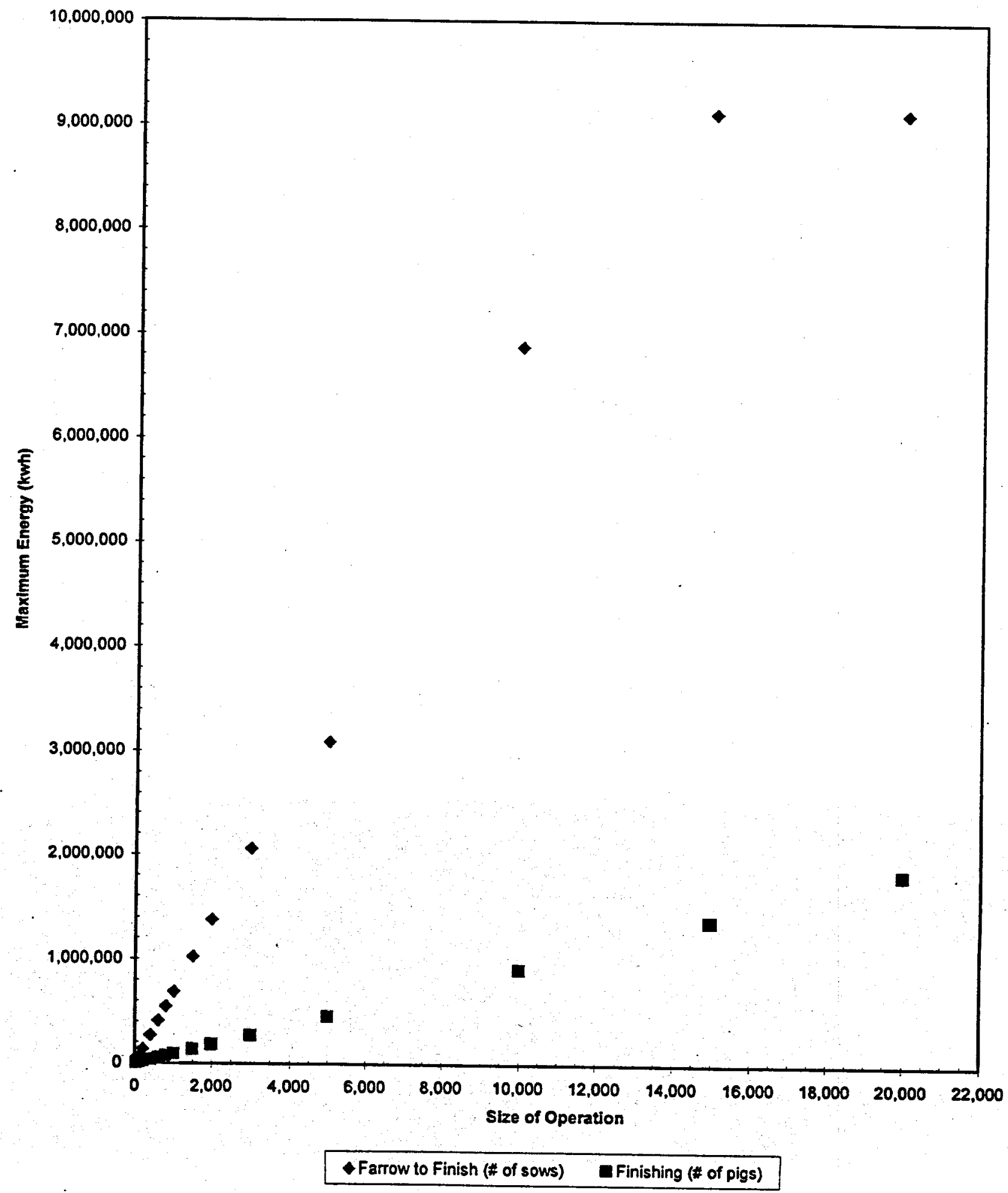

Figure 13: Swine Farrow to Finish and Finishing Maximum Energy Production 
Dairy Tie Stall with Plug Flow Digestor

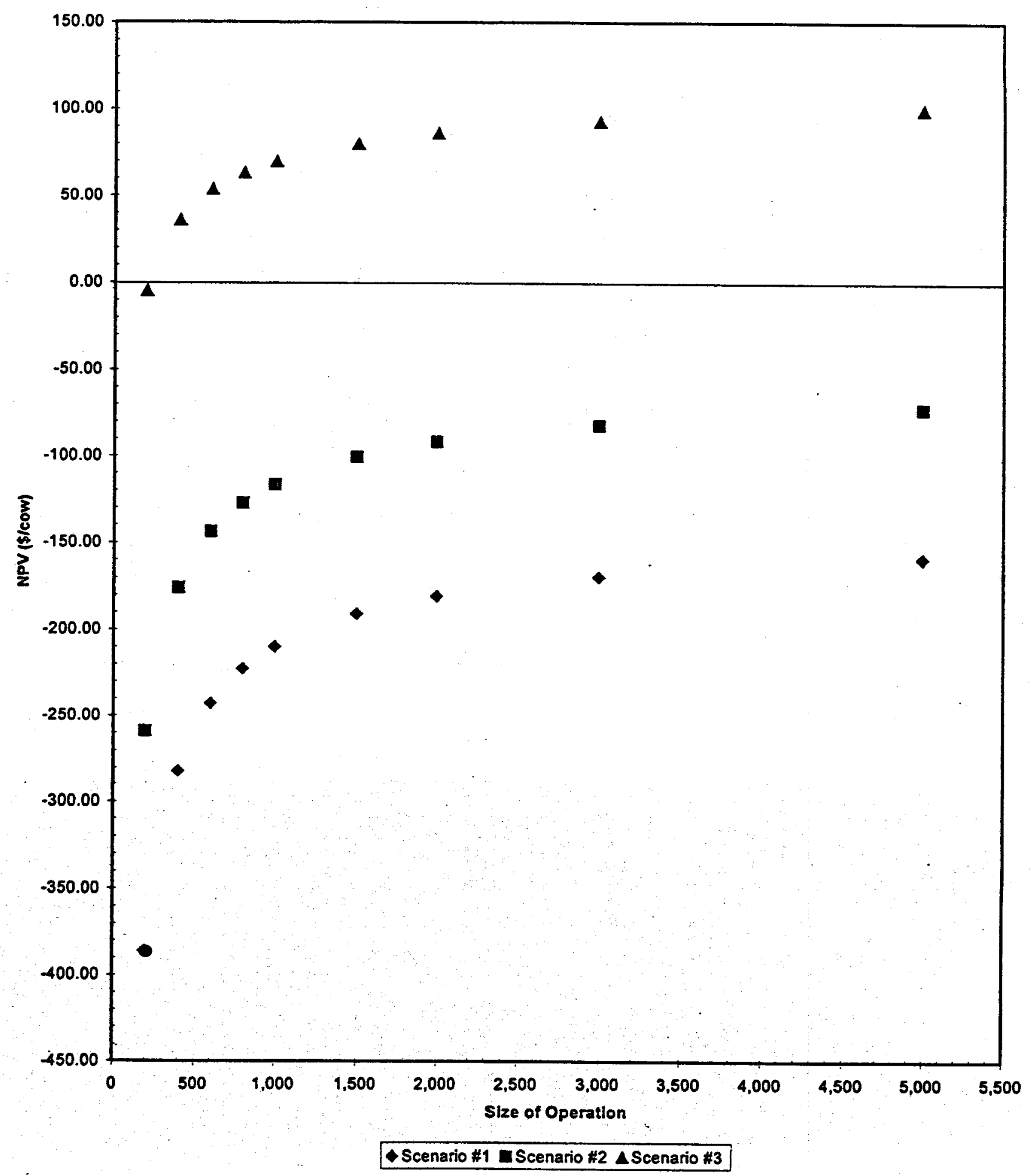

Figure 14: Net Present Value for Dairy Tie Stall with Plug Flow Digester 
Dairy Tie Stall with Plug Flow Digestor

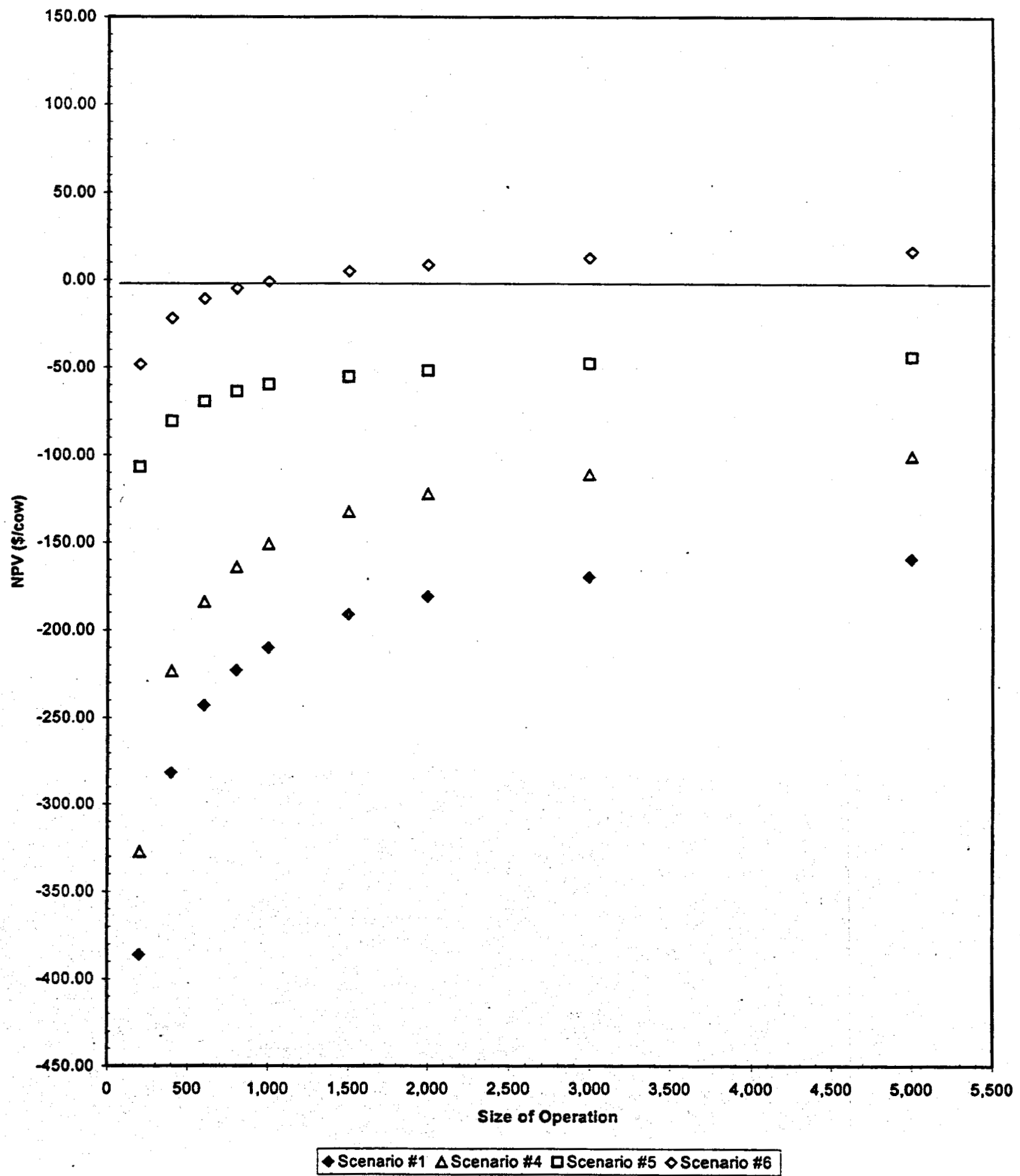

Figure 15: Net Present Value Dairy Tie Stall with Plug Flow Digester 
Dairy Tie Stall with Complete Mix Digester

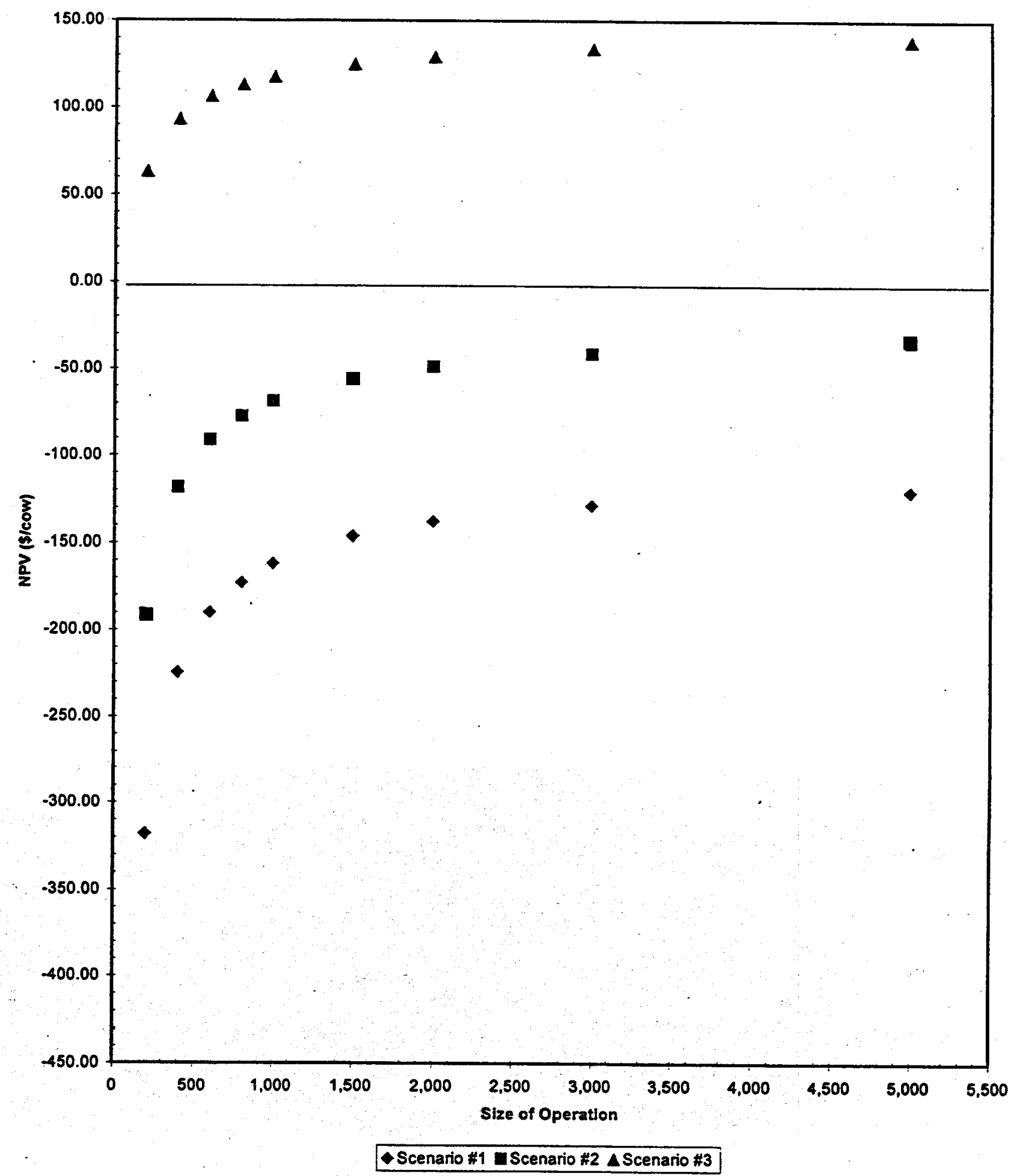

Figure 16: Net Present Value for Dairy Tie Stall with Complete Mix Digester 
Dairy Tie Stall with Complete Mix Digester

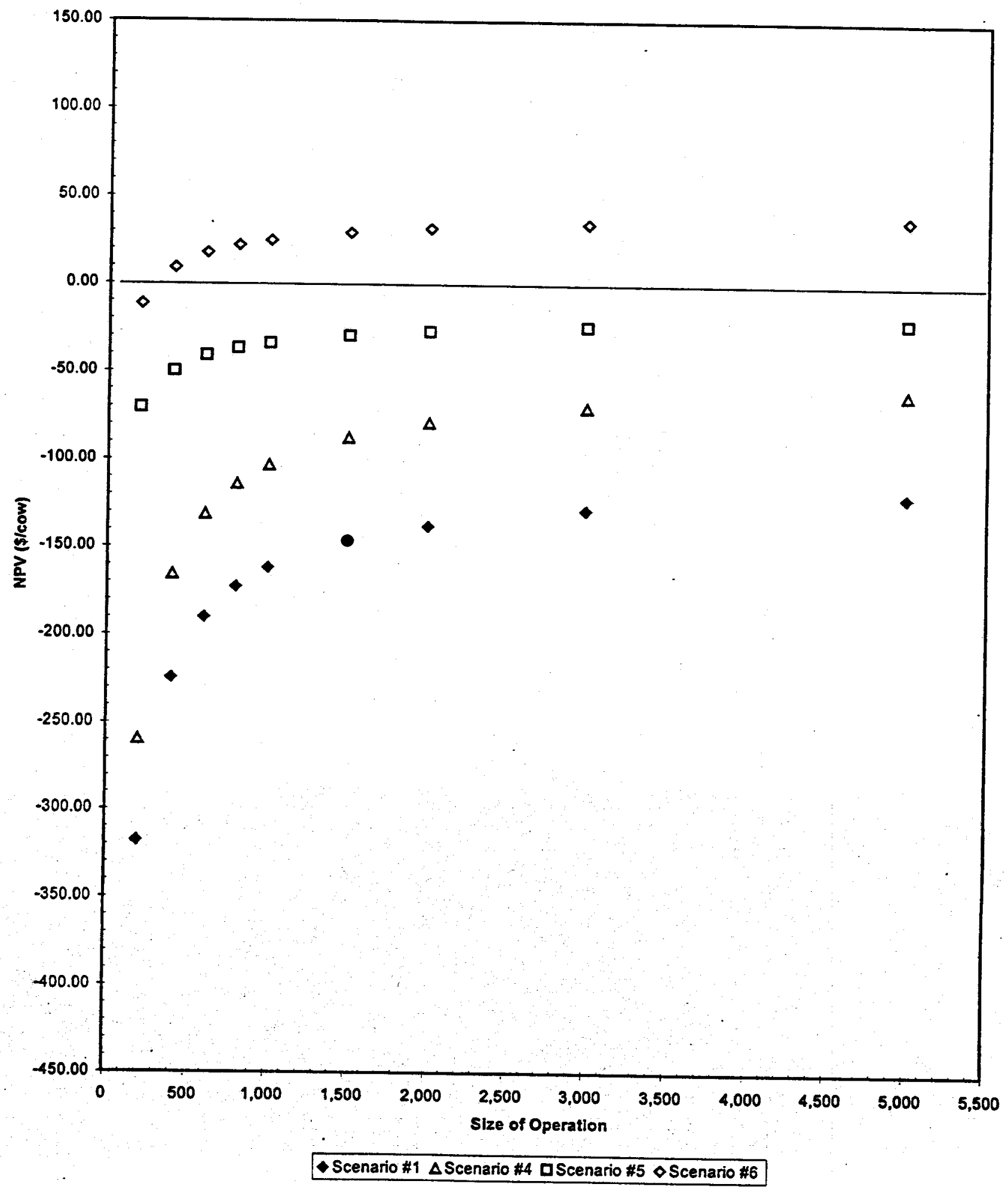

Figure 17: Net Present Value for Dairy Tie Stall with Complete Mix Digester 
Dairy Free Stall with Plug Flow Digestor

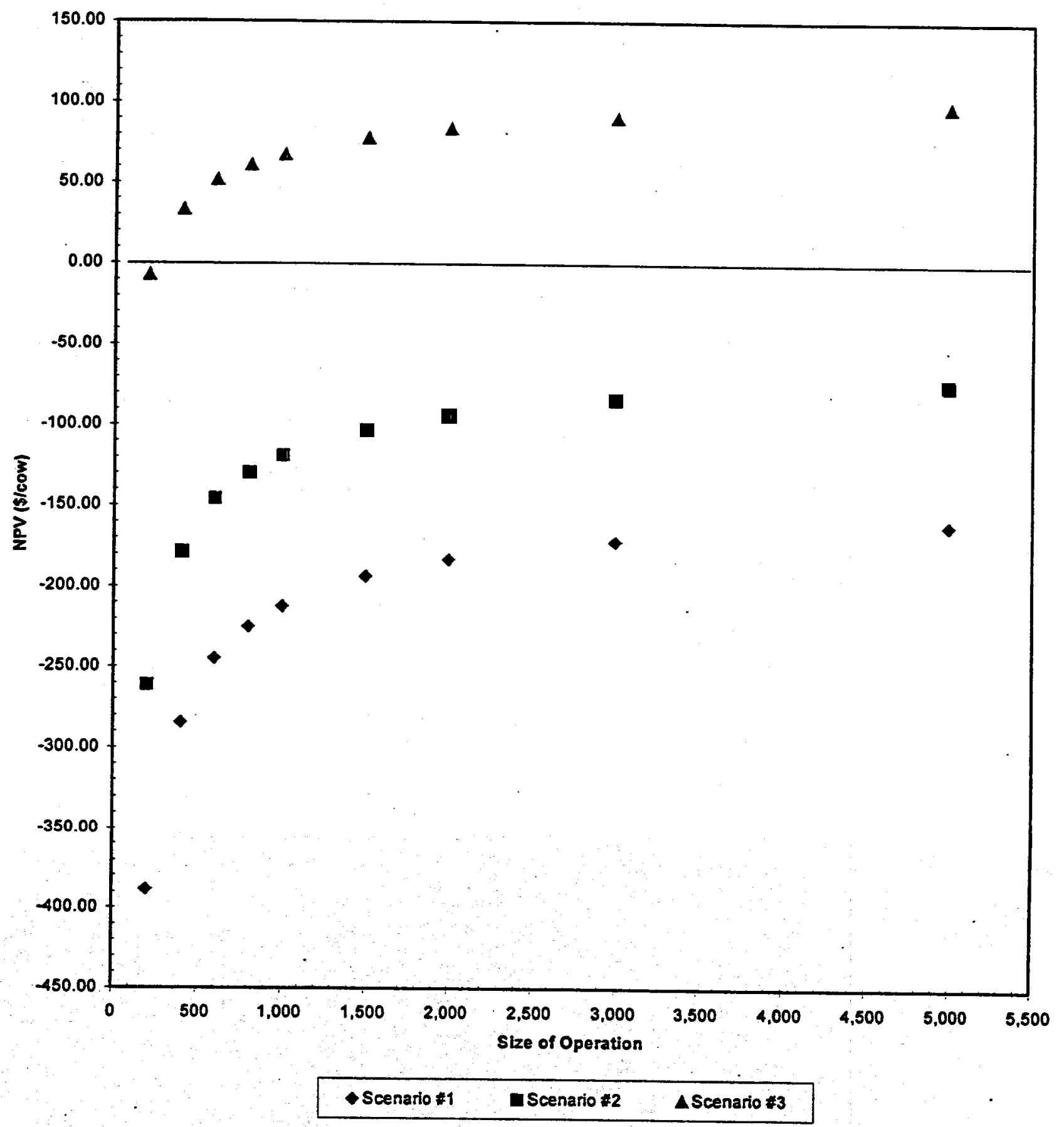

Figure 18: Net Present Value for Dairy Free Stall with Plug Flow Digester 
Free Stall with Plug Flow Digestor

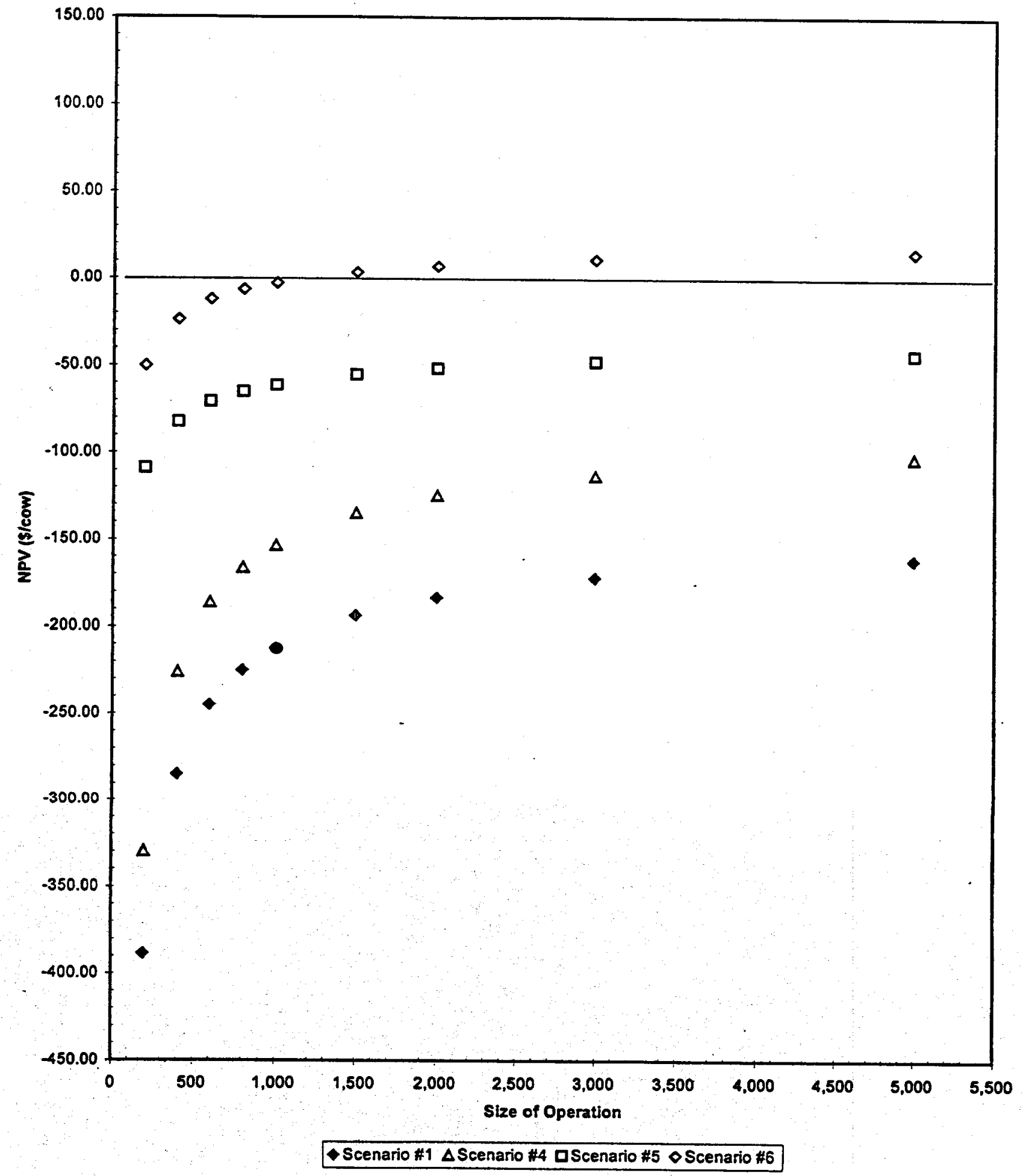

Figure 19: Net Present Value for Dairy Free Stall with Plug Flow Digester 
Dairy Free Stall with Complete Mix Digestor

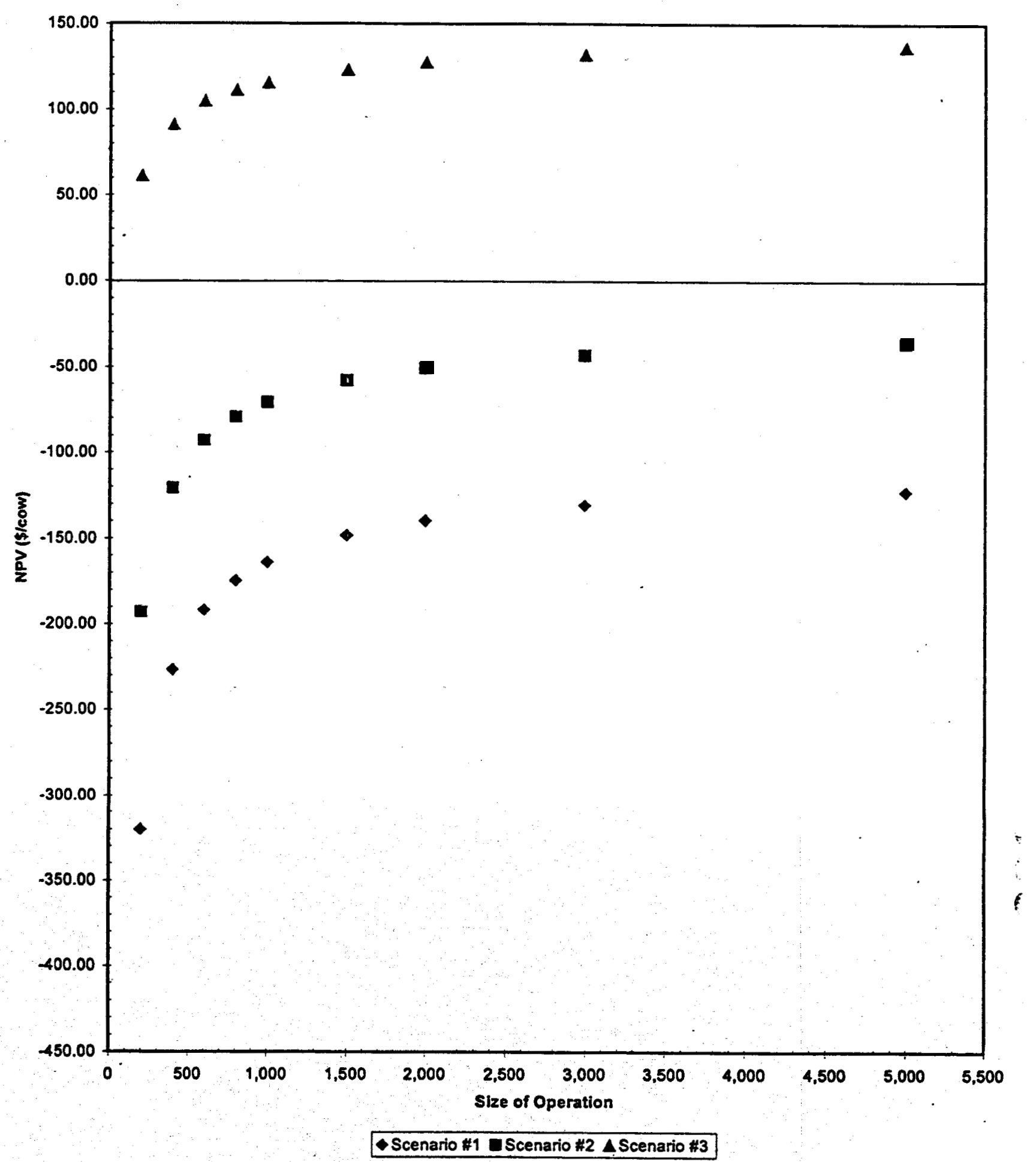

Figure 20: Net Present Value for Dairy Free Stall with Complete Mix Digester 
Dairy Free Stall with Complete Mix Digestor

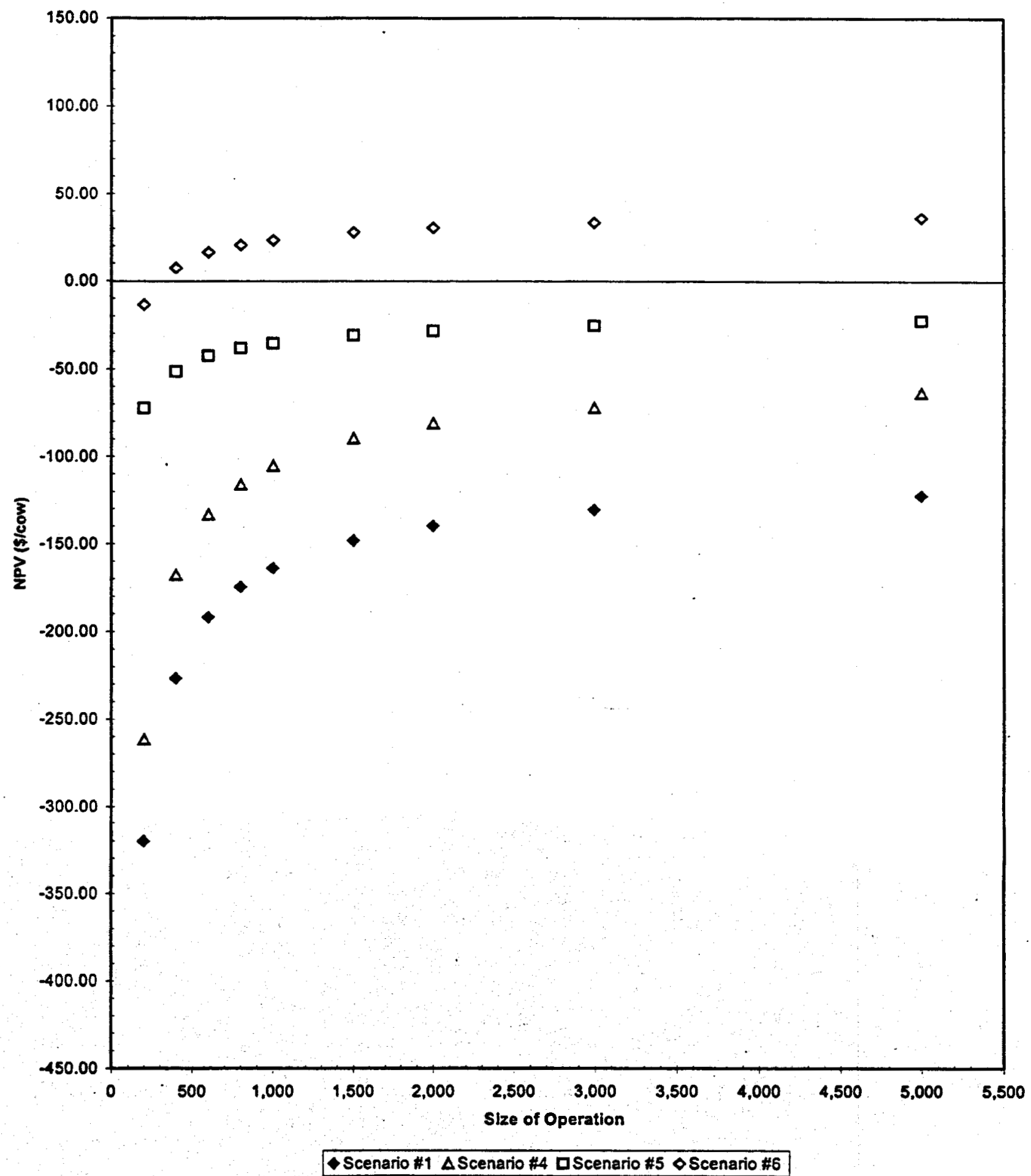

Figure 21: Net Present Value for Dairy Free Stall with Complete Mix Digester 
Dairy Tie \& Free Stall Manure Production

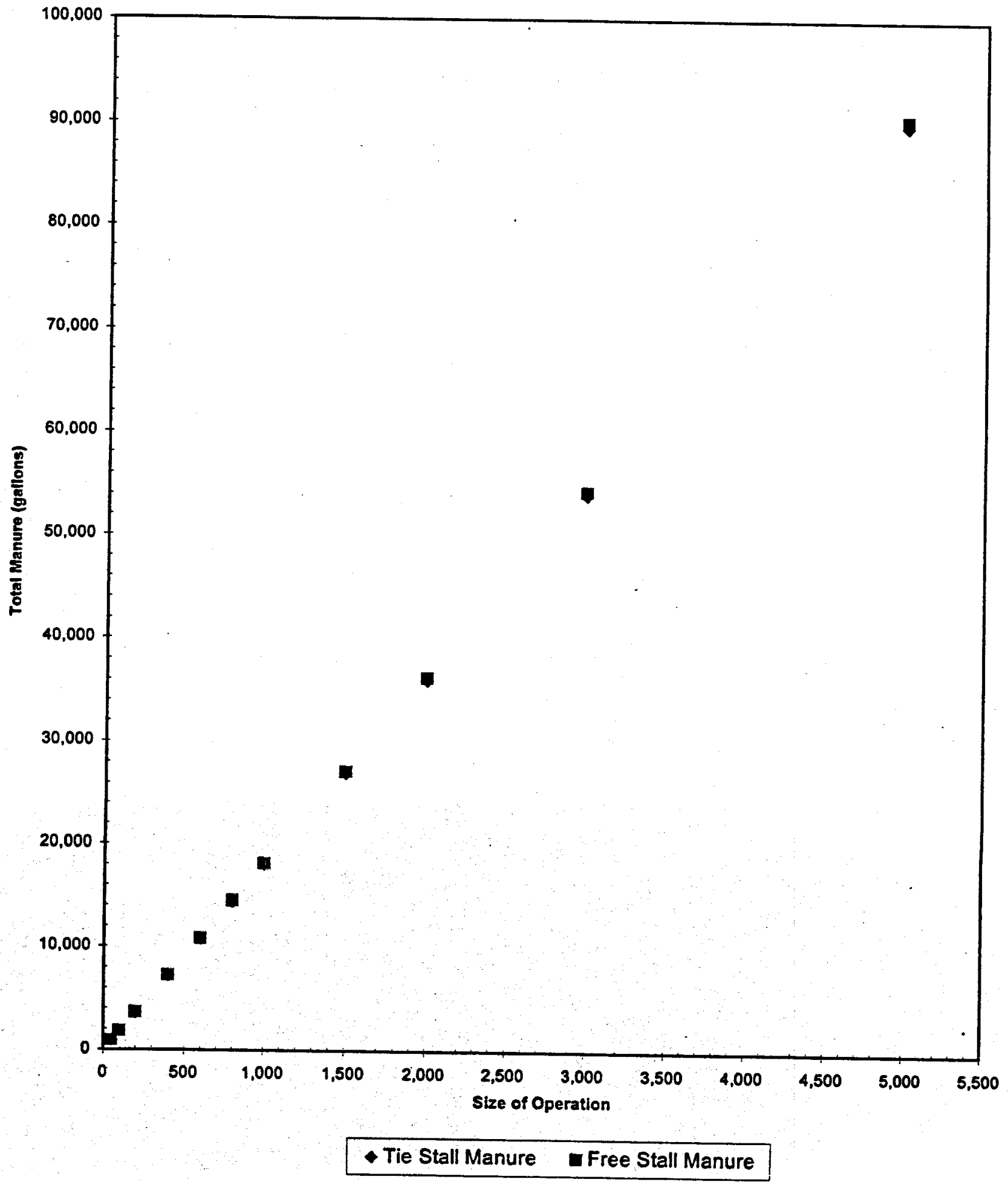

Figure 22: Diary Tie and Free Stall Manure Production 
Cost for Tie Stall with Plug Flow Digester

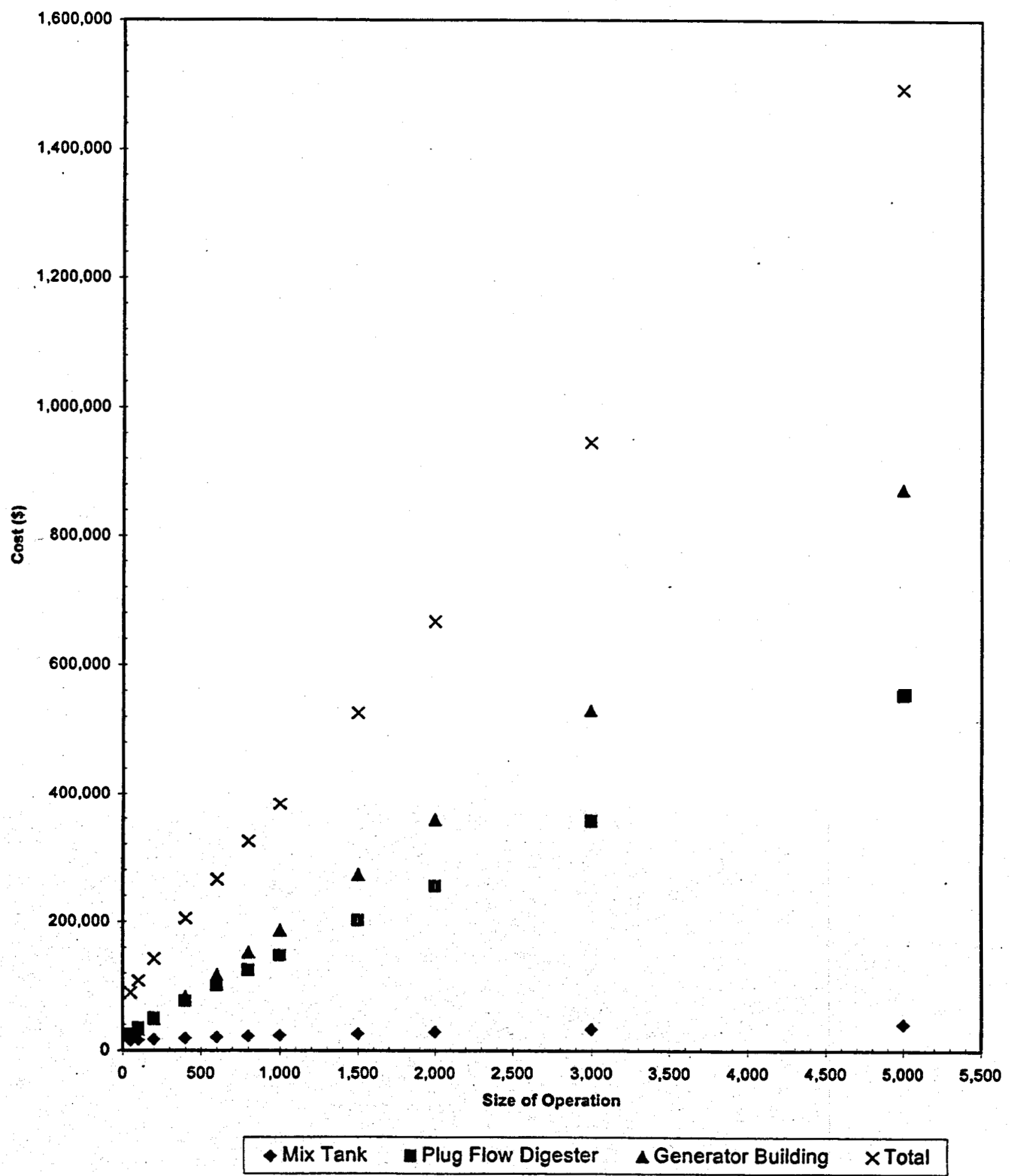

Figure 23: Dairy Tie Stall Plug Flow Digester Component and Total Costs 
Cost for Tie Stall with Complete Mix Digester

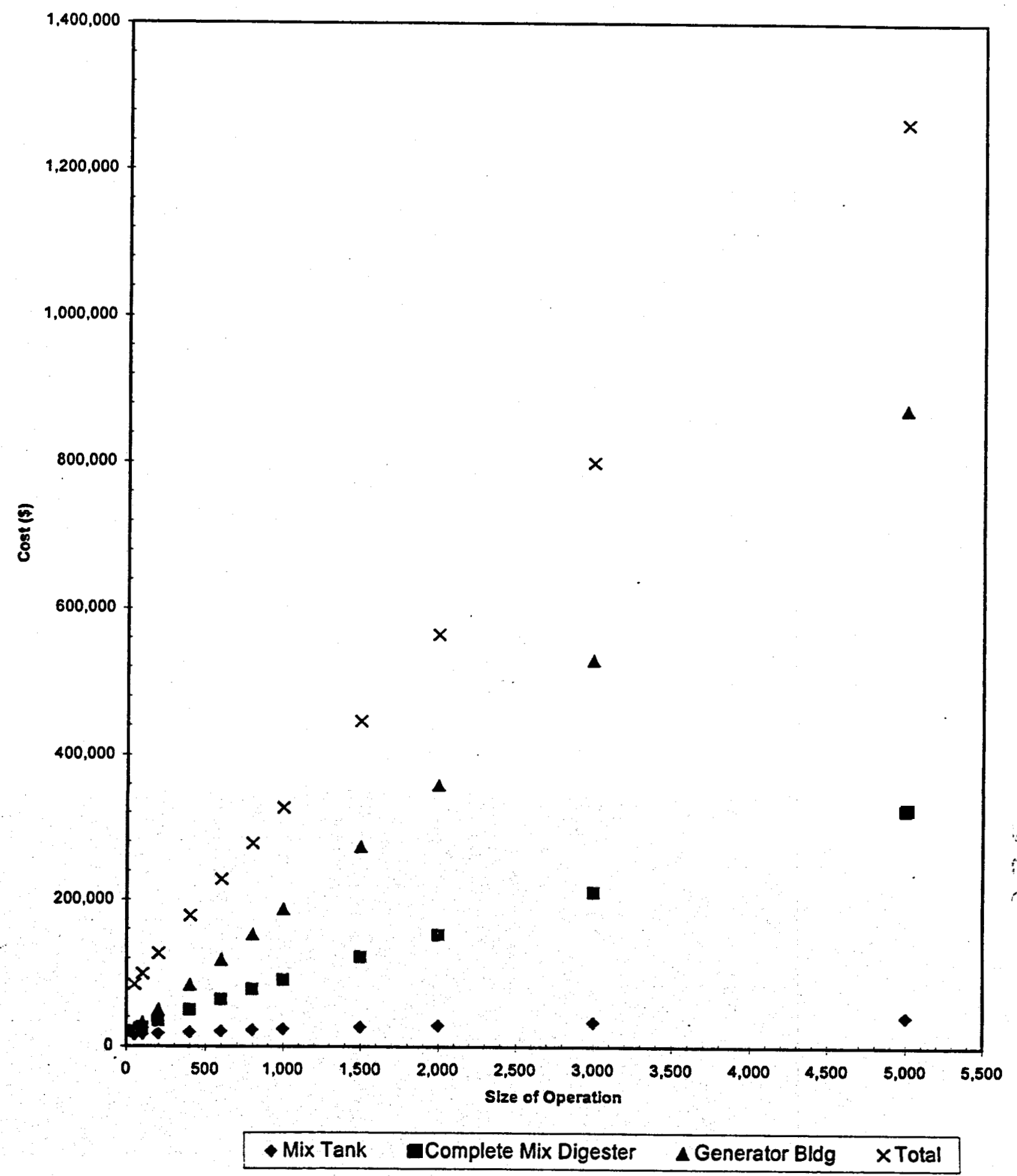

Figure 24: Dairy Tie Stall Complete Mix Digester Component and Total Costs 


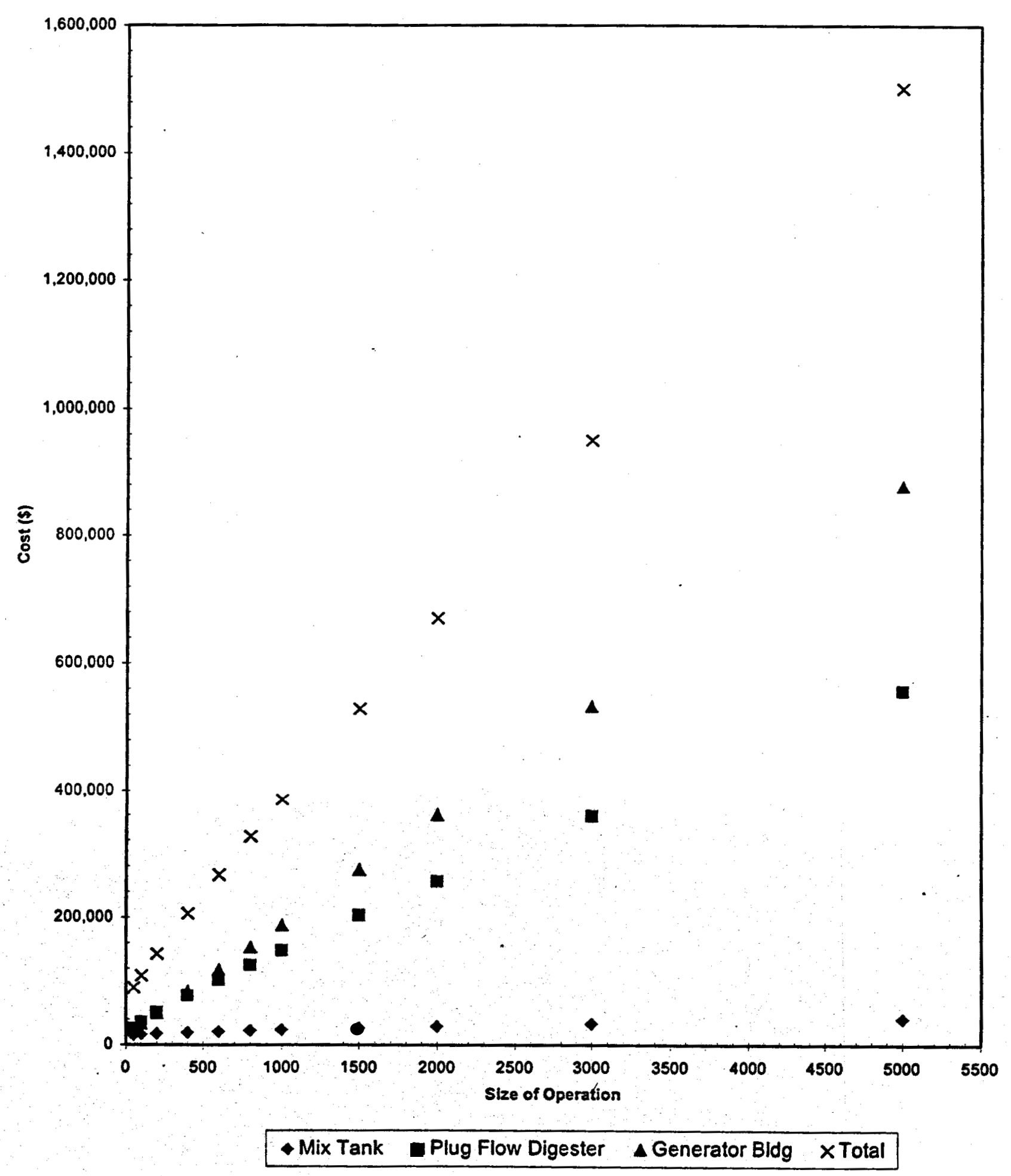

Figure 25: Dairy Free Stall Plug Flow Digester Component and Total Costs 
Cost for Free Stall with Complete Mix Digester

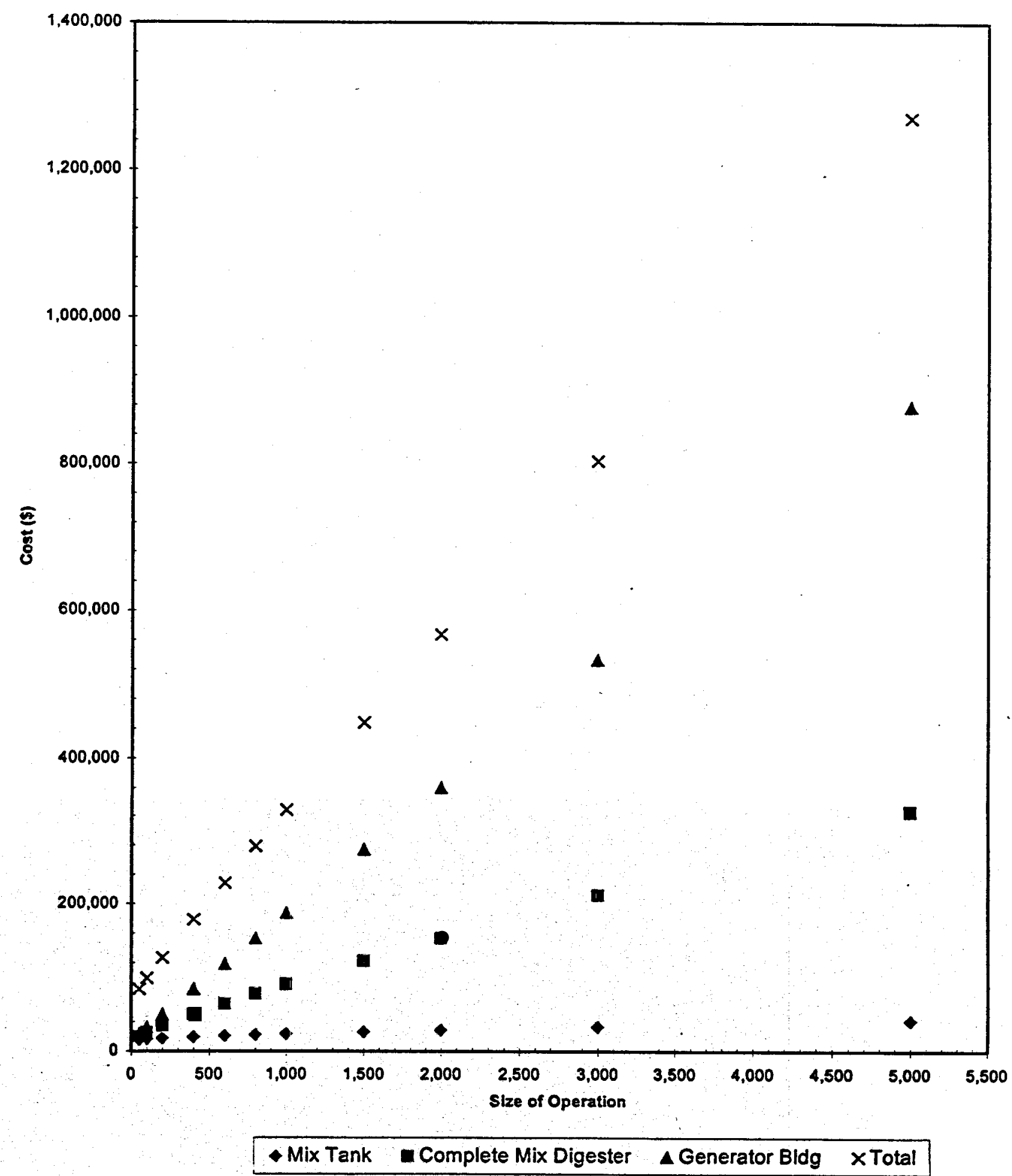

Figure 26: Dairy Free Stall Complete Mix Digester Component and Total Costs 
Dairy System Gas Production

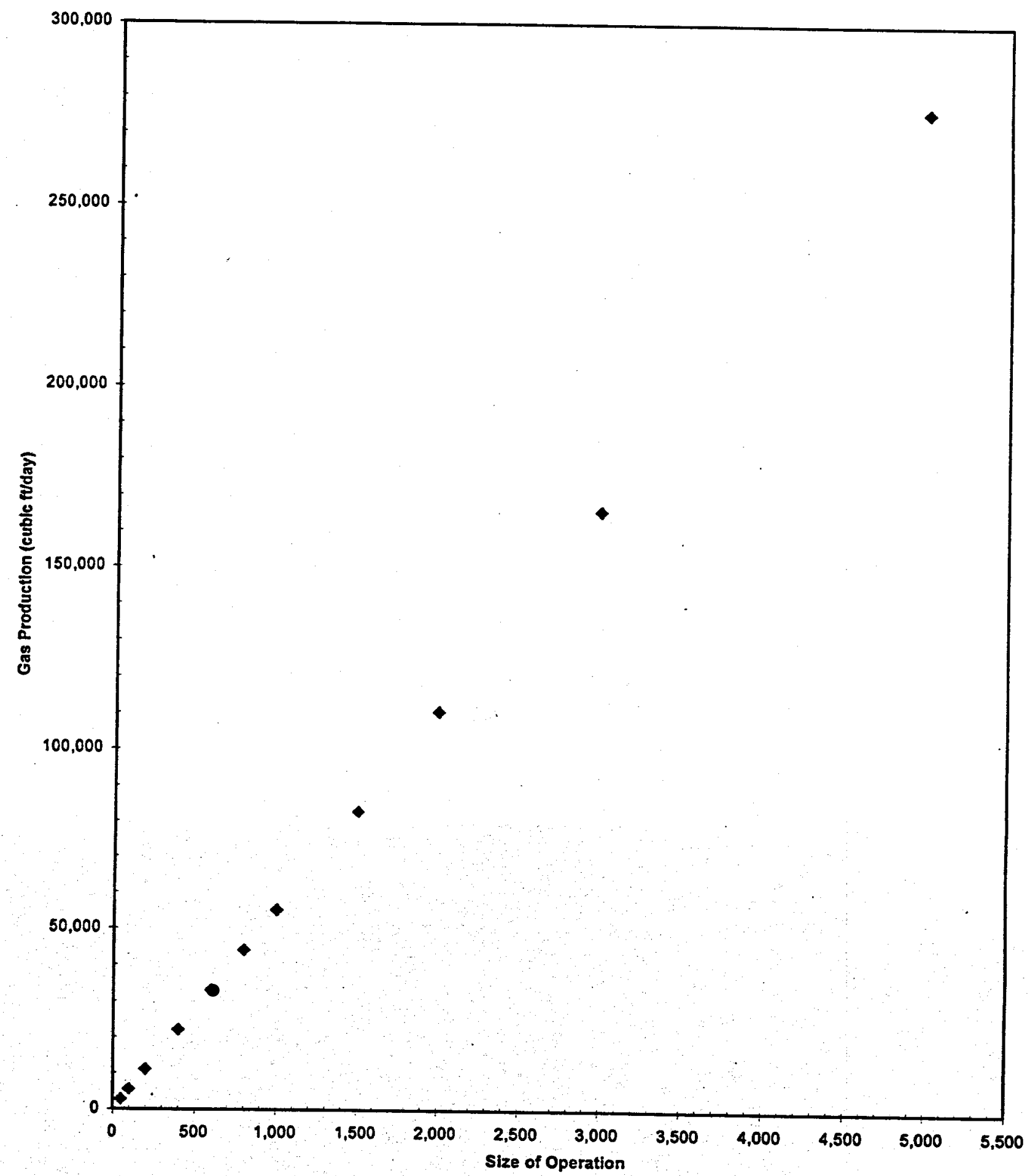

Figure 27: Dairy System Gas Production 
Dairy System Maximum Electricity Production

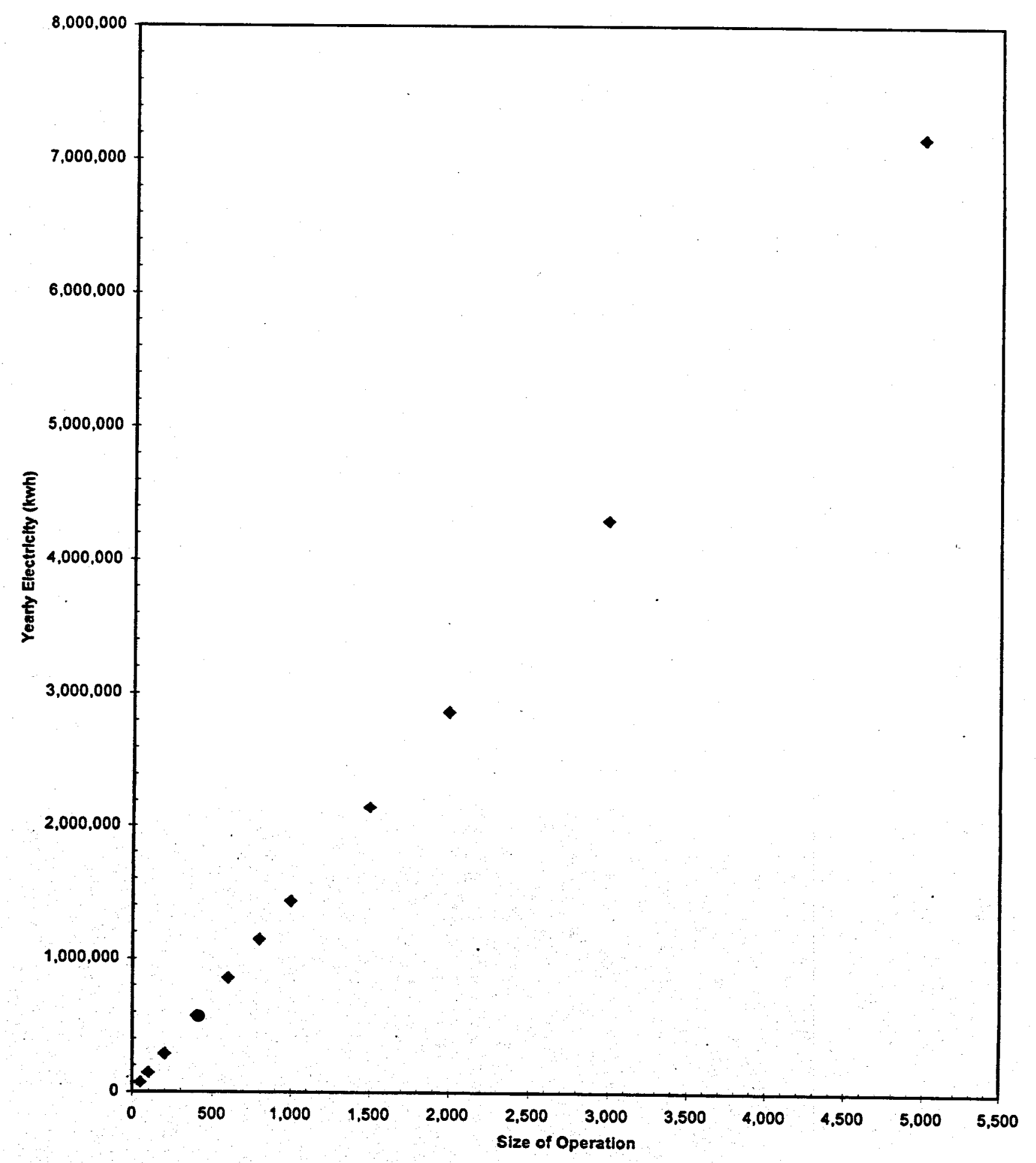

Figure 28: Dairy System Maximum Energy Production 


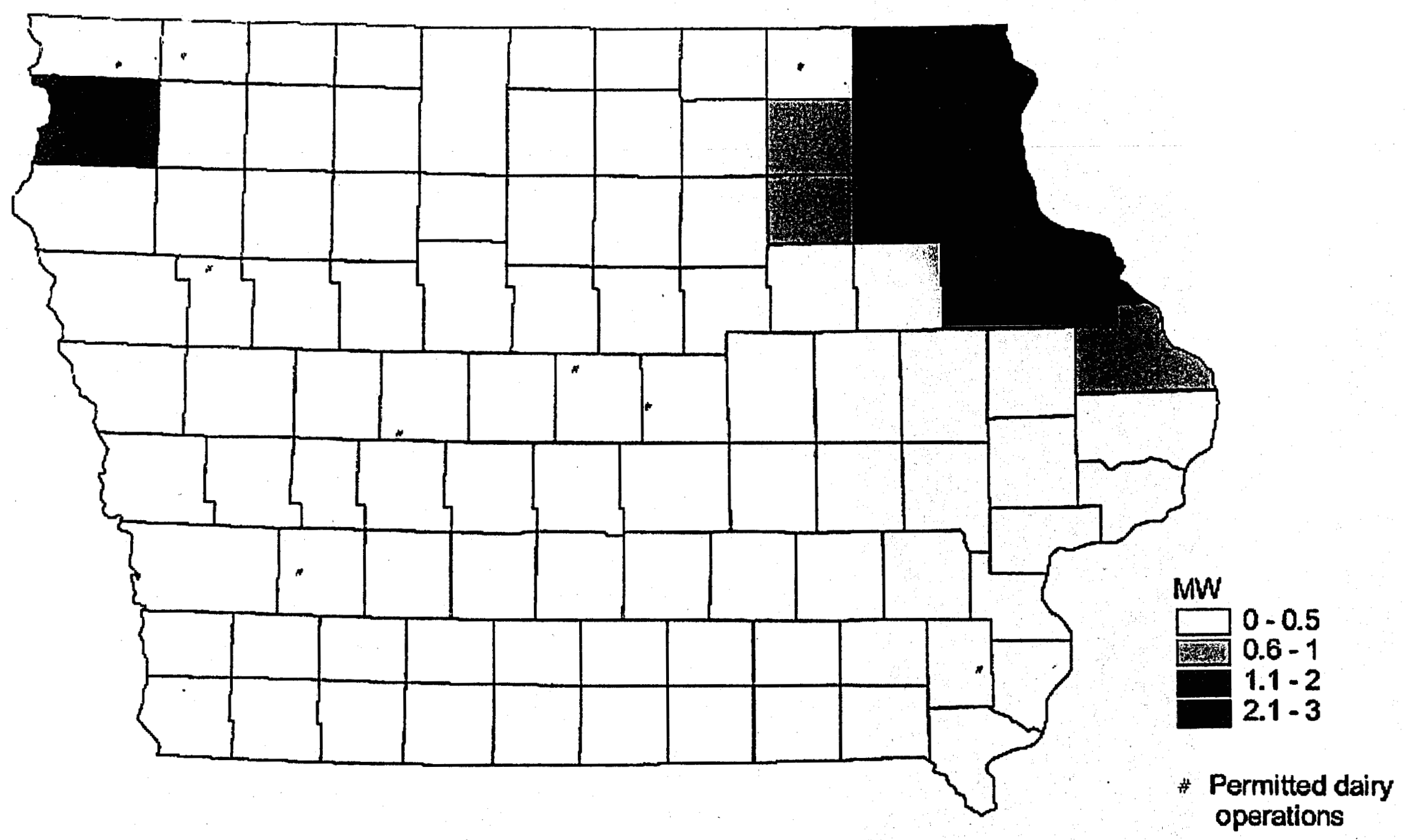

Figure 29: Electricity output (MW) per county if all available dairy manure generated methane is combusted in an engine generator ( $30 \%$ efficiency) to generate electricity. 


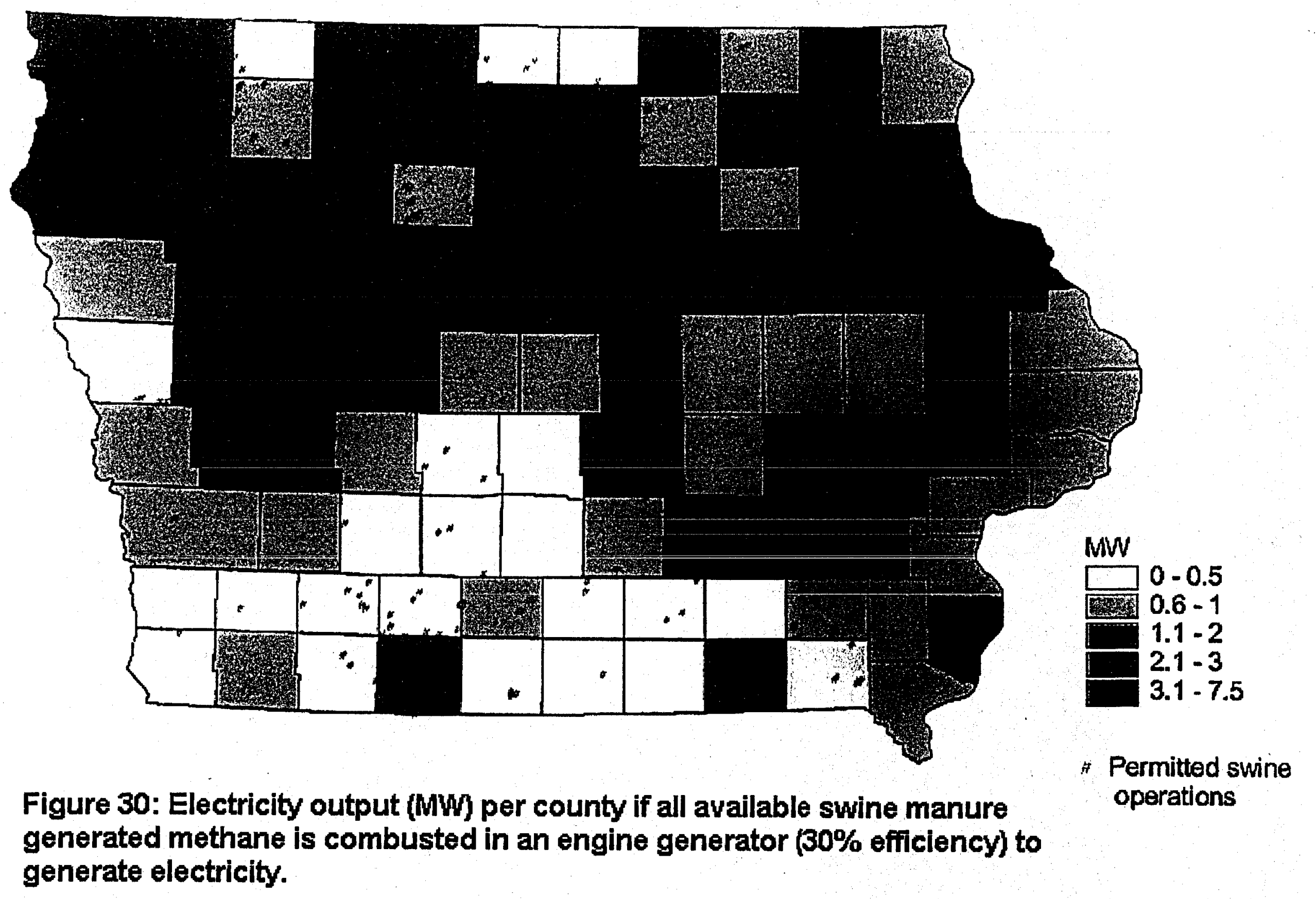




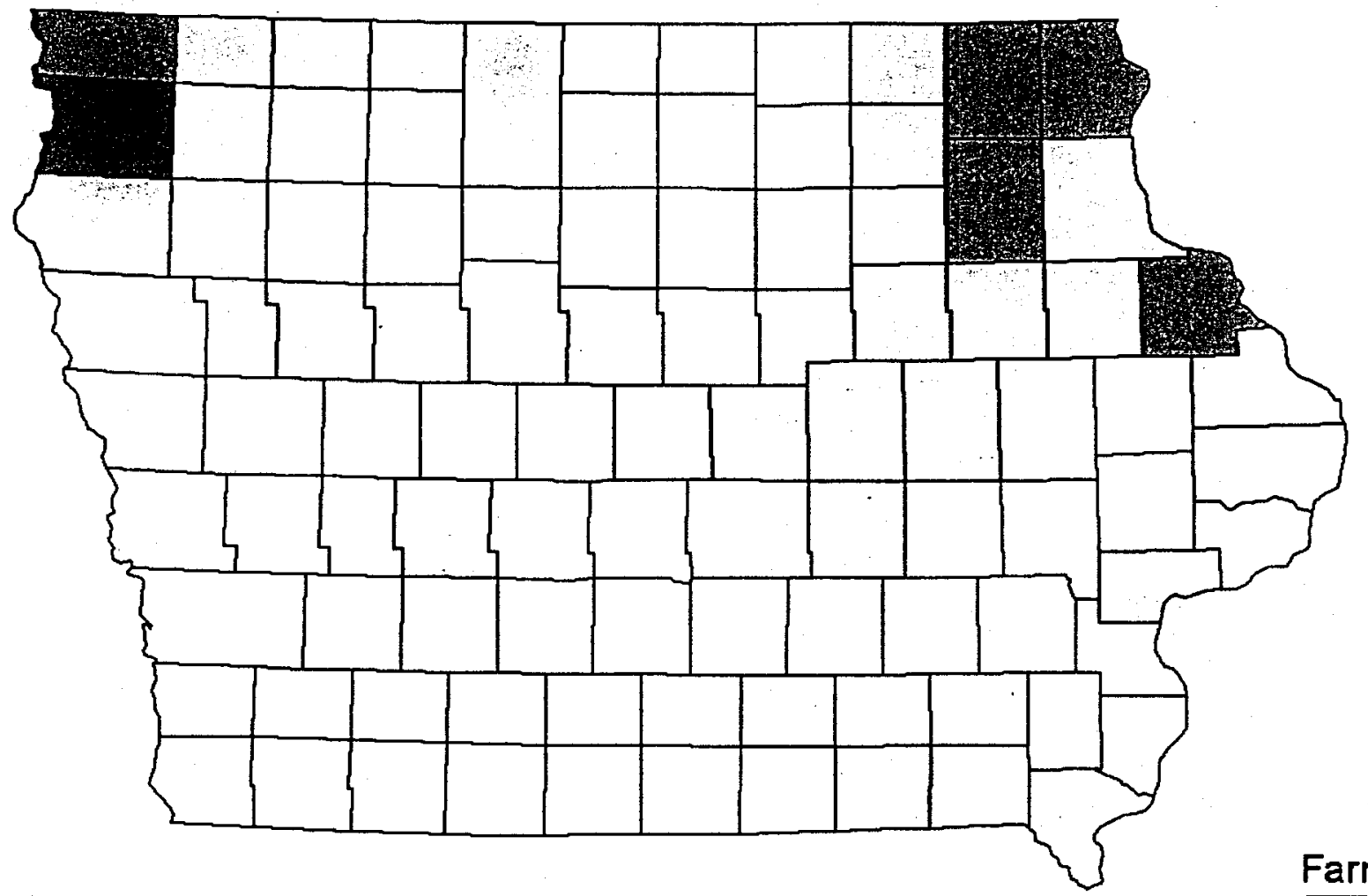

Figure 31: Maximum Number of Farms that Meet Dairy Herd Size Requirements in Table $\mathbf{4 5}$ for Scenario \#3 (Top) and Scenario \#6 (Bottom)

Farm Numbers
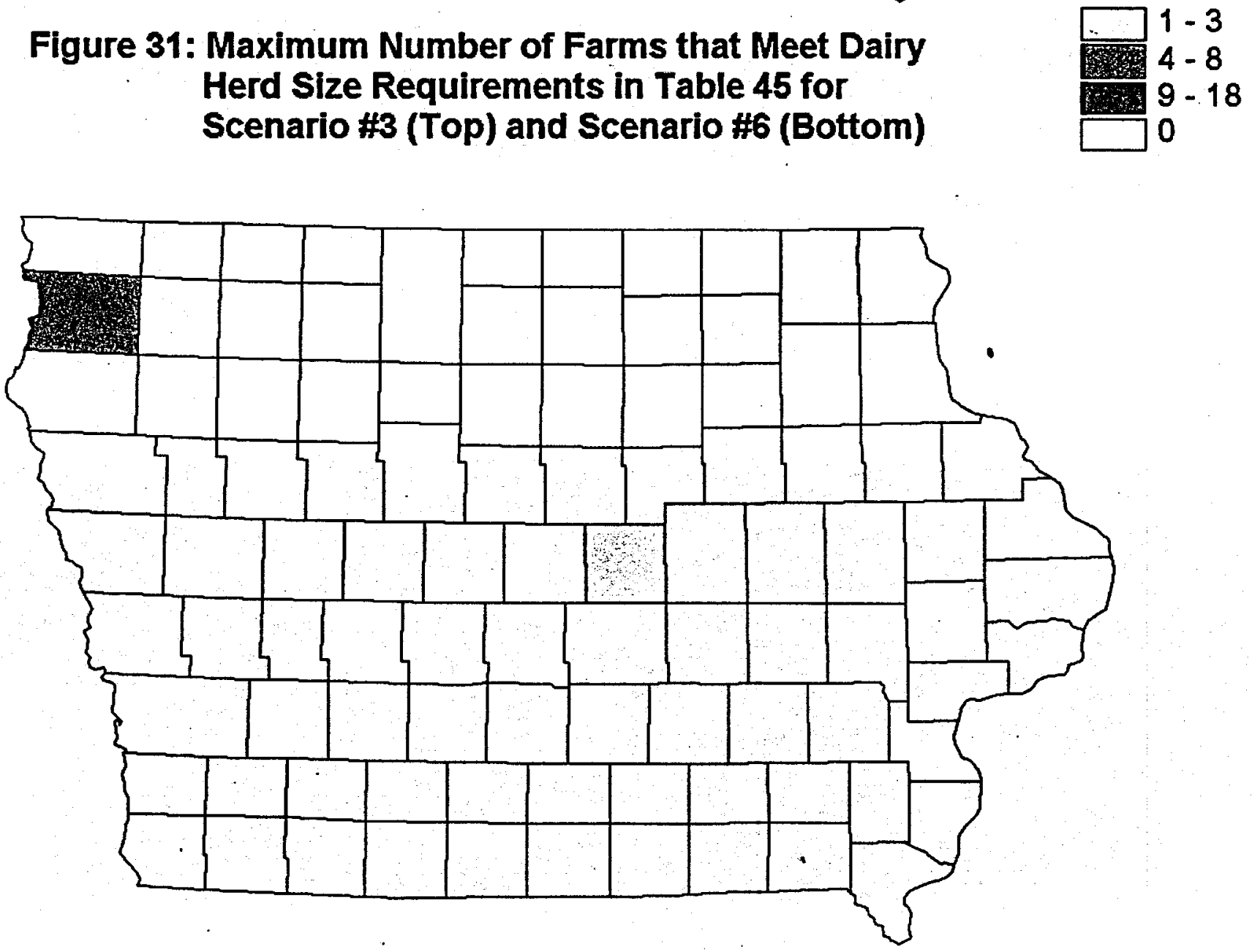


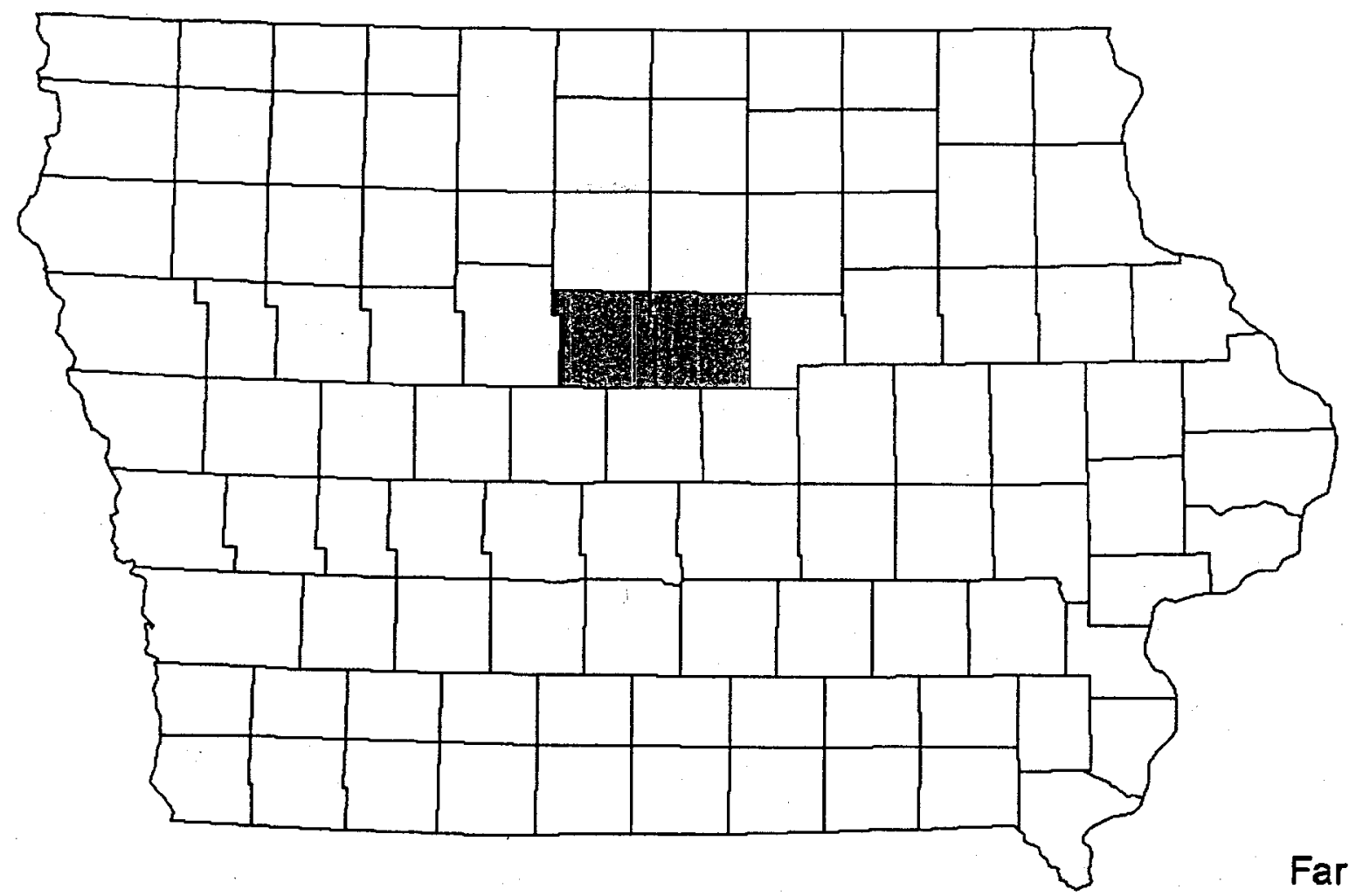

Figure 32: Maximum Number of Farms that Meet Swine Herd Size Requirements in Table 45 for Scenario \#3 (Top) and Scenario \#6 (Bottom)

Farm Numbers

$3-38$

$39-96$

$97-219$ 0

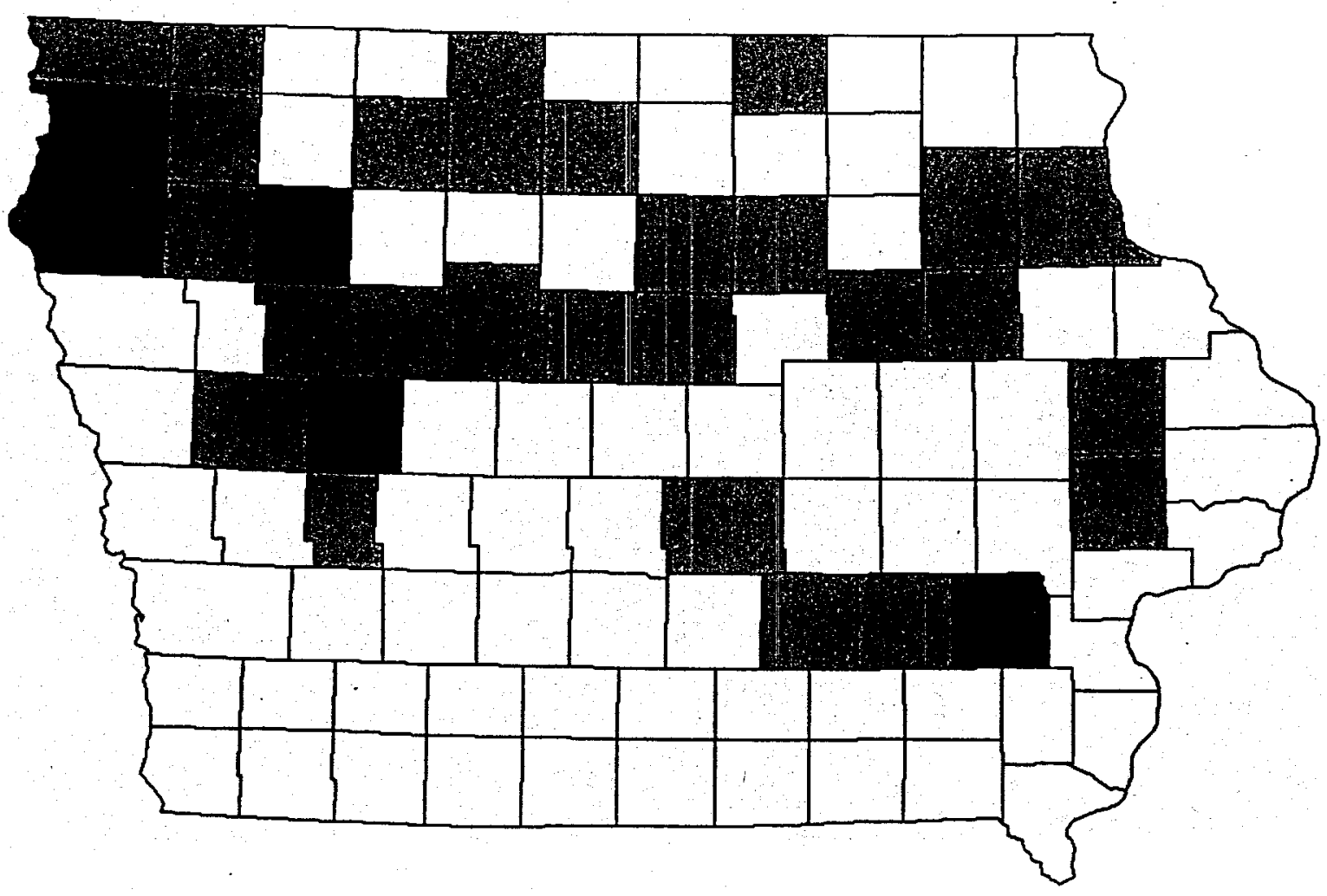


Appendix C 


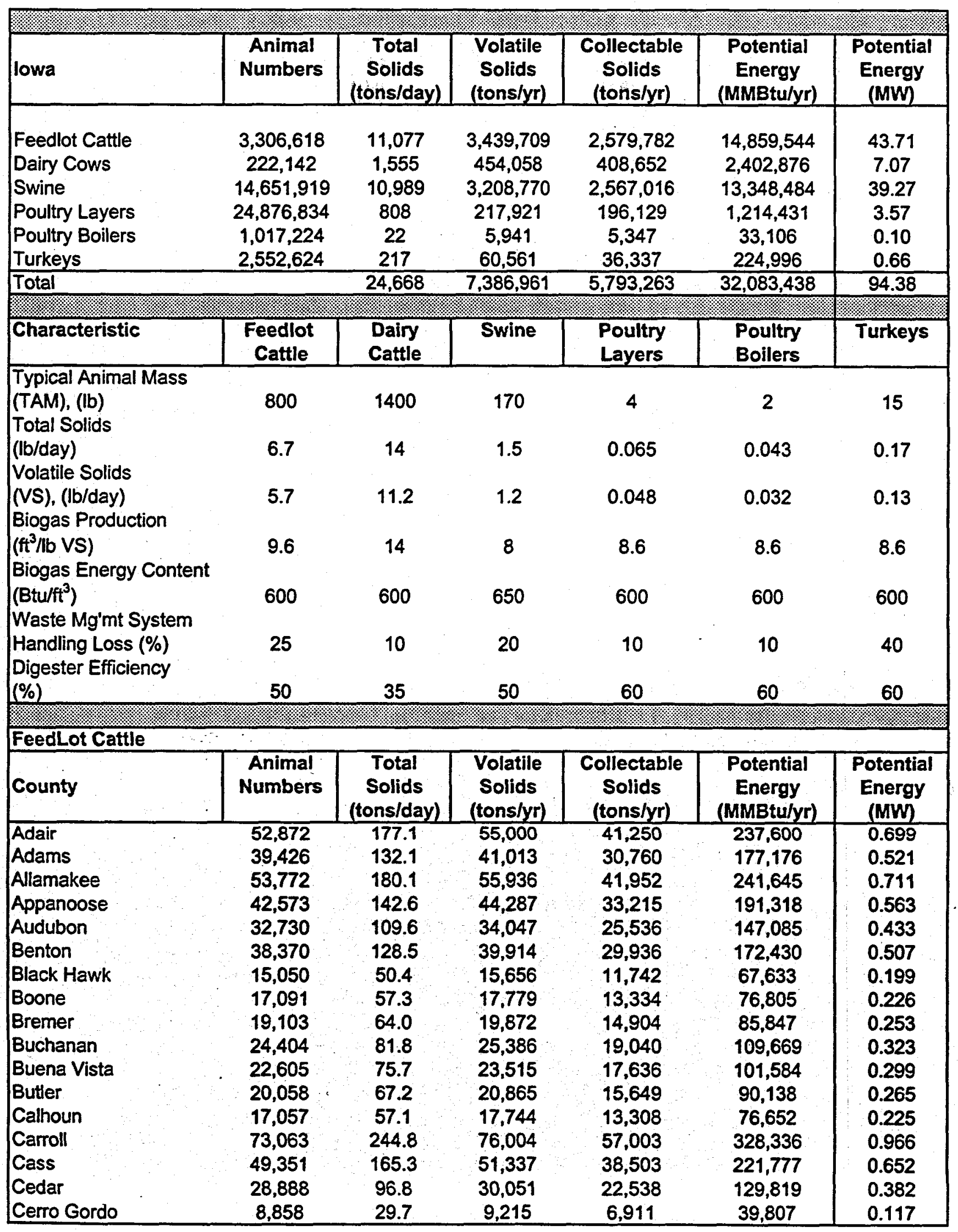




\begin{tabular}{|c|c|c|c|c|c|c|c|}
\hline County & & $\begin{array}{c}\text { Animal } \\
\text { Numbers }\end{array}$ & $\begin{array}{c}\text { Total } \\
\text { Solids } \\
\text { (tons/day) }\end{array}$ & $\begin{array}{c}\text { Volatile } \\
\text { Solids } \\
\text { (tons/yr) }\end{array}$ & $\begin{array}{c}\text { Collectable } \\
\text { Solids } \\
\text { (tons/yr) }\end{array}$ & $\begin{array}{c}\text { Potential } \\
\text { Energy } \\
\text { (MMBtulyr) }\end{array}$ & $\begin{array}{c}\text { Potential } \\
\text { Energy } \\
\text { (MW) }\end{array}$ \\
\hline Cherokee & & 47,783 & 160.1 & 49,706 & 37,280 & 214,731 & 0.632 \\
\hline Chickasaw & & 33,433 & 112.0 & 34,779 & 26,084 & 150,244 & 0.442 \\
\hline Clarke & & 40,364 & 135.2 & 41,989 & 31,491 & 181,391 & 0.534 \\
\hline Clay & $\cdot$ & 11,217 & 37.6 & 11,668 & 8,751 & 50,408 & 0.148 \\
\hline Clayton & & 61,395 & 205.7 & 63,866 & 47,900 & 275,902 & 0.812 \\
\hline Clinton & & 51,622 & 172.9 & 53,700 & 40,275 & 231,983 & 0.682 \\
\hline Crawford & & 60,007 & 201.0 & 62,422 & 46,817 & 269,664 & 0.793 \\
\hline Dallas & & 16,123 & 54.0 & 16,772 & 12,579 & 72,455 & 0.213 \\
\hline Davis & & 45,627 & 152.9 & 47,463 & 35,598 & 205,042 & 0.603 \\
\hline Decatur & & 61,391 & 205.7 & 63,862 & 47,896 & 275,884 & 0.812 \\
\hline Delaware & & 51,555 & 172.7 & 53,630 & 40,223 & 231,682 & 0.682 \\
\hline Des Moines & & 14,823 & 49.7 & 15,420 & 11,565 & 66,613 & 0.196 \\
\hline Dickinson & & 26,260 & 88.0 & 27,317 & 20,488 & 118,009 & 0.347 \\
\hline Dubuque & & 84,146 & 281.9 & 87,533 & 65,650 & 378,142 & 1.112 \\
\hline Emmet & & 15,161 & 50.8 & 15,771 & 11,828 & 68,132 & 0.200 \\
\hline Fayette & & 46,490 & 155.7 & 48,361 & 36,271 & 208,920 & 0.615 \\
\hline Floyd & & 14,958 & 50.1 & 15,560 & 11,670 & 67,219 & 0.198 \\
\hline Franklin & & 18,816 & 63.0 & 19,573 & 14,680 & 84,557 & 0.249 \\
\hline Fremont & & 15,843 & 53.1 & 16,481 & 12,361 & 71,197 & 0.209 \\
\hline Greene & & 21,033 & 70.5 & 21,880 & 16,410 & 94,520 & 0.278 \\
\hline Grundy & & 19,098 & 64.0 & 19,867 & 14,900 & 85,824 & 0.252 \\
\hline Guthrie & & 36,439 & 122.1 & 37,906 & 28,429 & 163,752 & 0.482 \\
\hline Hamilton & & 4,810 & 16.1 & 5,004 & 3,753 & 21,616 & 0.064 \\
\hline Hancock & & 11,261 & 37.7 & 11,714 & 8,786 & 50,606 & 0.149 \\
\hline Hardin & & 19,364 & 64.9 & 20,143 & 15,108 & 87,019 & 0.256 \\
\hline Harrison & & 33,609 & 112.6 & 34,962 & 26,221 & 151,035 & 0.444 \\
\hline Henry & & 17,711 & 59.3 & 18,424 & 13,818 & 79,591 & 0.234 \\
\hline Howard & & 26,090 & 87.4 & 27,140 & 20,355 & 117,245 & 0.345 \\
\hline Humboldt & & 11,637 & 39.0 & 12,105 & 9,079 & 52,295 & 0.154 \\
\hline Ida & & 23,638 & 79.2 & 24,589 & 18,442 & 106,226 & 0.312 \\
\hline lowa & & 43,389 & 145.4 & $45,135^{\circ}$ & 33,852 & 194,985 & 0.574 \\
\hline Jackson & & 81,725 & 273.8 & 85,014 & 63,761 & 367,262 & 1.080 \\
\hline Jasper & & 45,159 & 151.3 & 46,977 & 35,232 & 202,939 & 0.597 \\
\hline Jefferson & & 19,756 & 66.2 & 20,551 & 15,413 & 88,781 & 0.261 \\
\hline Johnson & & 35,052 & 117.4 & 36,463 & 27,347 & 157,519 & 0.463 \\
\hline Jones & & 57,783 & 193.6 & 60,109 & 45,082 & 259,670 & 0.764 \\
\hline Keokuk & & 28,398 & 95.1 & 29,541 & 22,156 & 127,617 & 0.375 \\
\hline Kossuth & & 29,240 & 98.0 & 30,417 & 22,813 & 131,401 & 0.387 \\
\hline Lee & & 25,437 & 85.2 & 26,461 & 19,846 & 114,311 & 0.336 \\
\hline Linn & & 34,757 & 116.4 & 36,156 & 27,117 & 156,194 & 0.459 \\
\hline Louisa & & 11,076 & 37.1 & 11,522 & 8,641 & 49,774 & 0.146 \\
\hline Lucas & & 40,267 & 134.9 & 41,888 & 31,416 & 180,955 & 0.532 \\
\hline Lyon & & 74,152 & 248.4 & 77,137 & 57,852 & 333,230 & 0.980 \\
\hline Madison & & 44,683 & 149.7 & 46,481 & 34,861 & 200,800 & 0.591 \\
\hline Mahaska & & 40,315 & 135.1 & 41,938 & 31,453 & 181,171 & 0.533 \\
\hline Marion & & 30,030 & 100.6 & 31,239 & 23,429 & 134,951 & 0.397 \\
\hline Marshall & & 24,558 & 82.3 & 25,546 & 19,160 & 110,361 & 0.325 \\
\hline Mills & & 14,090 & 47.2 & 14,657 & 10,993 & 63,319 & 0.186 \\
\hline
\end{tabular}




\begin{tabular}{|c|c|c|c|c|c|c|}
\hline County & $\begin{array}{c}\text { Animal } \\
\text { Numbers }\end{array}$ & $\begin{array}{c}\text { Total } \\
\text { Solids } \\
\text { (tons/day) }\end{array}$ & $\begin{array}{c}\text { Volatile } \\
\text { Solids } \\
\text { (tons/yr) }\end{array}$ & $\begin{array}{c}\text { Collectable } \\
\text { Solids } \\
\text { (tons/yr) }\end{array}$ & $\begin{array}{c}\text { Potential } \\
\text { Energy } \\
\text { (MMBtulyr) }\end{array}$ & $\begin{array}{l}\text { Potential } \\
\text { Energy } \\
\text { (MW) }\end{array}$ \\
\hline Mitchell & 41,639 & 139.5 & 43,315 & 32,486 & 187,121 & 0.550 \\
\hline Monona & 36,207 & $121: 3$ & 37,664 & 28,248 & 162,710 & 0.479 \\
\hline Monroe & 36,383 & 121.9 & 37,847 & 28,386 & 163,501 & 0.481 \\
\hline Montgomery & 29,110 & 97.5 & 30,282 & 22,711 & 130,817 & 0.385 \\
\hline Muscatine & 18,174 & 60.9 & 18,906 & 14,179 & 81,672 & 0.240 \\
\hline O'Brien & 33,304 & 111.6 & 34,644 & 25,983 & 149,664 & 0.440 \\
\hline Osceola & 29,588 & 99.1 & 30,779 & 23,084 & 132,965 & 0.391 \\
\hline Page & 39,386 & 131.9 & 40,971 & 30,728 & 176,996 & 0.521 \\
\hline Palo Alto & 18,481 & 61.9 & 19,225 & 14,419 & 83,051 & 0.244 \\
\hline Plymouth & 76,916 & 257.7 & 80,012 & 60,009 & 345,651 & 1.017 \\
\hline Pocahontas & 11,214 & 37.6 & 11,665 & 8,749 & 50,394 & 0.148 \\
\hline Polk & 9,528 & 31.9 & 9,912 & 7,434 & 42,818 & 0.126 \\
\hline Pottawattamie & 63,490 & 212.7 & 66,045 & 49,534 & 285,316 & 0.839 \\
\hline Poweshiek & 39,434 & 132.1 & 41,021 & 30,766 & 177,212 & 0.521 \\
\hline Ringgold & 41,731 & 139.8 & 43,411 & 32,558 & 187,534 & 0.552 \\
\hline Sac & 38,788 & 129.9 & 40,349 & 30,262 & 174,309 & 0.513 \\
\hline Scott & 19,639 & 65.8 & 20,429 & 15,322 & 88,255 & 0.260 \\
\hline Shelby & 40,019 & 134.1 & 41,630 & 31,222 & 179,841 & 0.529 \\
\hline Sioux & 159,839 & 535.5 & 166,273 & 124,704 & 718,297 & 2.113 \\
\hline Story & 15,623 & 52.3 & 16,252 & 12,189 & 70,208 & 0.207 \\
\hline Tama & 32,621 & 109.3 & 33,934 & 25,450 & 146,595 & 0.431 \\
\hline Taylor & 36,510 & 122.3 & 37,980 & 28,485 & 164,072 & 0.483 \\
\hline Union & 19,442 & 65.1 & 20,225 & 15,168 & 87,370 & 0.257 \\
\hline Van Buren & 29,728 & 99.6 & 30,925 & 23,193 & 133,594 & 0.393 \\
\hline Wapello & 20,536 & 68.8 & 21,363 & 16,022 & 92,286 & 0.271 \\
\hline Warren & 38,988 & 130.6 & 40,557 & 30,418 & 175,207 & 0.515 \\
\hline Washington & 24,167 & 81.0 & 25,140 & 18,855 & 108,604 & 0.319 \\
\hline Wayne & 42,003 & 140.7 & 43,694 & 32,770 & 188,756 & 0.555 \\
\hline Webster & 12,578 & 42.1 & 13,084 & 9,813 & 56,524 & 0.166 \\
\hline Winnebago & 5,461 & 18.3 & 5,681 & $-4,261$ & 24,541 & 0.072 \\
\hline Winneshiek & 61,741 & 206.8 & 64,226 & 48,170 & 277,457 & 0.816 \\
\hline $\begin{array}{l}\text { Woodbury } \\
\text { Worth }\end{array}$ & $\begin{array}{c}62,525 \\
9,425\end{array}$ & $\begin{array}{c}209.5 \\
31.6\end{array}$ & $\begin{array}{c}65,042 \\
9,804\end{array}$ & $\begin{array}{c}48,781 \\
7,353\end{array}$ & $\begin{array}{c}280,980 \\
42,355\end{array}$ & $\begin{array}{l}0.827 \\
0.125\end{array}$ \\
\hline Wright & 5,181 & 17.4 & 5,390 & 4,042 & 23,283 & 0.068 \\
\hline \multicolumn{7}{|l|}{ Dairy Cattle } \\
\hline County & $\begin{array}{c}\text { Animal } \\
\text { Numbers }\end{array}$ & $\begin{array}{c}\text { Total } \\
\text { Solids } \\
\text { (tons/day) }\end{array}$ & $\begin{array}{l}\text { Volatile } \\
\text { Solids } \\
\text { (tons/yr) }\end{array}$ & $\begin{array}{c}\text { Collectable } \\
\text { Solids } \\
\text { (tons/yr) }\end{array}$ & $\begin{array}{c}\text { Potential } \\
\text { Energy } \\
\text { (MMBtulyr) }\end{array}$ & $\begin{array}{l}\text { Potential } \\
\text { Energy } \\
\text { (MW) }\end{array}$ \\
\hline Adair & 296 & 2.1 & 605 & 545 & 3,202 & 0.009 \\
\hline Adams & 177 & 1.2 & 362 & 326 & 1,915 & 0.006 \\
\hline Allamakee & 15,173 & 106.2 & 31,014 & 27,912 & 164,124 & 0.483 \\
\hline Appanoose & 398 & 2.8 & 814 & 732 & 4,305 & 0.013 \\
\hline Audubon & 190 & 1.3 & 388 & 350 & 2,055 & 0.006 \\
\hline Benton & 2,029 & 14.2 & 4,147 & 3,733 & 21,947 & 0.065 \\
\hline Black Hawk & 1,491 & 10.4 & 3,048 & 2,743 & 16,128 & 0.047 \\
\hline Boone & 104 & 0.7 & 213 & 191 & 1,125 & 0.003 \\
\hline
\end{tabular}




\begin{tabular}{|c|c|c|c|c|c|c|}
\hline County & $\begin{array}{c}\text { Animal } \\
\text { Numbers }\end{array}$ & $\begin{array}{c}\text { Total } \\
\text { Solids } \\
\text { (tons/day) }\end{array}$ & $\begin{array}{c}\text { Volatile } \\
\text { Solids } \\
\text { (tons/yr) }\end{array}$ & $\begin{array}{l}\text { Collectable } \\
\text { Solids } \\
\text { (tons/yr) }\end{array}$ & $\begin{array}{l}\text { Potential } \\
\text { Energy } \\
\text { (MMBtu/yr) }\end{array}$ & $\begin{array}{l}\text { Potential } \\
\text { Energy } \\
\text { (MW) }\end{array}$ \\
\hline Bremer & 5,398 & 37.8 & 11,034 & 9,930 & 58,389 & 0.172 \\
\hline Buchanan & 3,785 & 26.5 & 7,737 & 6,963 & 40,942 & 0.120 \\
\hline Buena Vista & 321 & 2.2 & 656 & 591 & 3,472 & 0.010 \\
\hline Butler & 1,827 & 12.8 & 3,734 & 3,361 & 19,762 & 0.058 \\
\hline Calhoun & 0 & 0.0 & 0 & 0 & 0 & 0.000 \\
\hline Carroll & 233 & 1.6 & 476 & 429 & 2,520 & 0.007 \\
\hline Cass & 363 & 2.5 & 742 & 668 & 3,927 & 0.012 \\
\hline Cedar & 899 & 6.3 & 1,838 & 1,654 & 9,724 & 0.029 \\
\hline Cerro Gordo & 560 & 3.9 & 1,145 & 1,030 & 6.057 & 0.018 \\
\hline Cherokee & 804 & 5.6 & 1,643 & 1,479 & 8,697 & 0.026 \\
\hline Chickasaw & 5,674 & 39.7 & 11,598 & 10,438 & 61,375 & 0.181 \\
\hline Clarke & 357 & 2.5 & 730 & 657 & 3,862 & 0.011 \\
\hline Clay & 0 & 0.0 & 0 & 0 & 0 & 0.000 \\
\hline Clayton & 21,965 & 153.8 & 44,896 & 40,407 & 237,592 & 0.699 \\
\hline Clinton & 2,482 & 17.4 & 5,073 & 4,566 & 26,847 & 0.079 \\
\hline Crawford & 668 & 4.7 & 1,365 & 1,229 & 7,226 & 0.021 \\
\hline Dallas & 98 & 0.7 & 200 & 180 & 1,060 & 0.003 \\
\hline Davis & 1,834 & 12.8 & 3,749 & 3,374 & 19,838 & 0.058 \\
\hline Decatur & 232 & 1.6 & 474 & 427 & 2,510 & 0.007 \\
\hline Delaware & 18,844 & 131.9 & 38,517 & 34,665 & 203,833 & 0.600 \\
\hline Des Moines & 520 & 3.6 & 1,063 & 957 & 5,625 & 0.017 \\
\hline Dickinson & 586 & 4.1 & 1,198 & 1,078 & 6,339 & 0.019 \\
\hline Dubuque & 27,998 & 196.0 & 57,228 & 51,505 & 302,850 & 0.891 \\
\hline Emmet & 0 & 0.0 & 0 & 0 & 0 & 0.000 \\
\hline Fayette & 12,778 & 89.4 & 26,118 & 23,506 & 138,218 & 0.407 \\
\hline Floyd & 963 & 6.7 & 1,968 & 1,772 & 10,417 & 0.031 \\
\hline Franklin & 436 & 3.1 & 891 & 802 & 4,716 & 0.014 \\
\hline Fremont & 0 & 0.0 & 0 & 0 & 0 & 0.000 \\
\hline Greene & 8 & 0.1 & 16 & 15 & 87 & 0.000 \\
\hline Grundy & 745 & 5.2 & 1,523 & 1,371 & 8.059 & 0.024 \\
\hline Guthrie & 557 & 3.9 & 1,139 & 1,025 & 6,025 & 0.018 \\
\hline Hamilton & 340 & 2.4 & 695 & 625 & 3,678 & 0.011 \\
\hline Hancock & 355 & 2.5 & 726 & 653 & 3,840 & 0.011 \\
\hline Hardin & 161 & 1.1 & 329 & 296 & 1,742 & 0.005 \\
\hline Harrison & 84 & 0.6 & 172 & 155 & 909 & 0.003 \\
\hline Henry & 364 & 2.5 & 744 & 670 & 3,937 & 0.012 \\
\hline Howard & 4,561 & 31.9 & 9,323 & 8,390 & 49,336 & 0.145 \\
\hline Humboldt & 299 & 2.1 & 611 & 550 & 3,234 & 0.010 \\
\hline Ida & 0 & 0.0 & 0 & 0 & 0 & 0.000 \\
\hline lowa & 994 & 7.0 & 2,032 & 1,829 & 10,752 & 0.032 \\
\hline Jackson & 7,592 & 53.1 & 15,518 & 13,966 & 82,122 & 0.242 \\
\hline Jasper & 857 & 6.0 & 1,752 & 1,577 & 9,270 & 0.027 \\
\hline Jefferson & 524 & 3.7 & 1,071 & 964 & 5,668 & 0.017 \\
\hline Johnson & 2,938 & 20.6 & 6,005 & 5,405 & 31,780 & 0.093 \\
\hline Jones & 3,256 & 22.8 & 6,655 & 5,990 & 35,220 & 0.104 \\
\hline Keokuk & 203 & 1.4 & 415 & 373 & 2,196 & 0.006 \\
\hline Kossuth & 1,191 & 8.3 & 2,434 & 2,191 & 12,883 & 0.038 \\
\hline Lee & 1,447 & 10.1 & 2,958 & 2,662 & 15,652 & 0.046 \\
\hline
\end{tabular}




\begin{tabular}{|c|c|c|c|c|c|c|}
\hline County & $\begin{array}{c}\text { Animal } \\
\text { Numbers }\end{array}$ & $\begin{array}{c}\text { Total } \\
\text { Solids } \\
\text { (tons/day) }\end{array}$ & $\begin{array}{l}\text { Volatile } \\
\text { Solids } \\
\text { (tons/yr) }\end{array}$ & $\begin{array}{c}\text { Collectable } \\
\text { Solids } \\
\text { (tons/yr) }\end{array}$ & $\begin{array}{l}\text { Potential } \\
\text { Energy } \\
\text { (MMBtu/yr) }\end{array}$ & $\begin{array}{l}\text { Potential } \\
\text { Energy } \\
\text { (MW) }\end{array}$ \\
\hline Linn & 2,033 & 14.2 & 4,155 & 3,740 & 21,991 & 0.065 \\
\hline Louisa & 193 & 1.4 & 394 & 355 & 2,088 & 0.006 \\
\hline Lucas & 239 & 1.7 & 489 & 440 & 2,585 & 0.008 \\
\hline Lyon & 4,315 & 30.2 & 8,820 & 7,938 & 46,675 & 0.137 \\
\hline Madison & 12 & 0.1 & 25 & 22 & 130 & 0.000 \\
\hline Mahaska & 1,106 & 7.7 & 2,261 & 2,035 & 11,963 & 0.035 \\
\hline Marion & 742 & 5.2 & 1,517 & 1,365 & 8,026 & 0.024 \\
\hline Marshall & 855 & 6.0 & 1,748 & 1,573 & 9,248 & 0.027 \\
\hline Mills & 85 & 0.6 & 174 & 156 & 919 & 0.003 \\
\hline Mitchell & 2,996 & 21.0 & 6,124 & 5,511 & 32,407 & 0.095 \\
\hline Monona & 217 & 1.5 & 444 & 399 & 2,347 & 0.007 \\
\hline Monroe & 705 & 4.9 & 1,441 & 1,297 & 7,626 & 0.022 \\
\hline Montgomery & 247 & 1.7 & 505 & 454 & 2,672 & 0.008 \\
\hline Muscatine & 1,043 & 7.3 & 2,132 & 1,919 & 11,282 & 0.033 \\
\hline O'Brien & 760 & 5.3 & 1,553 & 1,398 & 8,221 & 0.024 \\
\hline Osceola & 1,338 & 9.4 & 2,735 & 2,461 & 14,473 & 0.043 \\
\hline Page & 75 & 0.5 & 153 & 138 & 811 & 0.002 \\
\hline Palo Alto & 362 & 2.5 & 740 & 666 & 3,916 & 0.012 \\
\hline Plymouth & 2,215 & 15.5 & 4,527 & 4,075 & 23,959 & 0.070 \\
\hline Pocahontas & 443 & 3.1 & 905 & 815 & 4,792 & 0.014 \\
\hline Polk & 246 & 1.7 & 503 & 453 & 2,661 & 0.008 \\
\hline Pottawattamie & 314 & 2.2 & 642 & 578 & 3,396 & 0.010 \\
\hline Poweshiek & 1,091 & 7.6 & 2,230 & 2,007 & 11,801 & 0.035 \\
\hline Ringgold & 242 & 1.7 & 495 & 445 & 2,618 & 0.008 \\
\hline Sac & 754 & 5.3 & 1,541 & 1,387 & 8,156 & 0.024 \\
\hline Scott & 844 & 5.9 & 1,725 & 1,553 & 9,129 & 0.027 \\
\hline Shelby & 324 & 2.3 & 662 & 596 & 3,505 & 0.010 \\
\hline Sioux & 14,214 & 99.5 & 29,053 & 26,148 & 153,751 & 0.452 \\
\hline Story & 1,342 & 9.4 & 2,743 & 2,469 & 14,516 & 0.043 \\
\hline Tama & 1,460 & 10.2 & 2,984 & 2,686 & 15,793 & 0.046 \\
\hline Taylor & 220 & 1.5 & 450 & 405 & 2,380 & 0.007 \\
\hline Union & 0 & 0.0 & 0 & 0 & 0 & 0.000 \\
\hline Van Buren & 941 & 6.6 & 1,923 & 1,731 & 10,179 & 0.030 \\
\hline Wapello & 439 & 3.1 & 897 & 808 & 4,749 & 0.014 \\
\hline Warren & 494 & 3.5 & 1,010 & 909 & 5,344 & 0.016 \\
\hline Washington & 1,272 & 8.9 & 2,600 & 2,340 & 13,759 & 0.040 \\
\hline Wayne & 479 & 3.4 & 979 & 881 & 5,181 & 0.015 \\
\hline Webster & 292 & 2.0 & 597 & 537 & 3,159 & 0.009 \\
\hline Winnebago & 533 & 3.7 & 1,089 & 981 & 5,765 & 0.017 \\
\hline Winneshiek & 19,617 & 137.3 & 40,097 & 36,087 & 212,194 & 0.624 \\
\hline Woodbury & 109 & 0.8 & 223 & 201 & 1,179 & 0.003 \\
\hline Worth & 467 & 3.3 & 955 & 859 & 5,051 & 0.015 \\
\hline Wright & 64 & 0.4 & 131 & 118 & 692 & 0.002 \\
\hline
\end{tabular}




\begin{tabular}{|c|c|c|c|c|c|c|}
\hline \multicolumn{7}{|l|}{ Swine } \\
\hline County & $\begin{array}{c}\text { Animal } \\
\text { Numbers }\end{array}$ & $\begin{array}{c}\text { Total } \\
\text { Solids } \\
\text { (tons/day) }\end{array}$ & $\begin{array}{c}\text { Volatile } \\
\text { Solids } \\
\text { (tons/yr) }\end{array}$ & $\begin{array}{l}\text { Collectable } \\
\text { Solids } \\
\text { (tons/yr) }\end{array}$ & $\begin{array}{l}\text { Potential } \\
\text { Energy } \\
\text { (MMBtu/yr) }\end{array}$ & $\begin{array}{c}\text { Potential } \\
\text { Energy } \\
\text { (MW) }\end{array}$ \\
\hline Adair & 39,032 & 29.3 & 8,548 & 6,838 & 35,560 & 0.105 \\
\hline Adams & 58,998 & 44.2 & 12,921 & 10,336 & 53,750 & 0.158 \\
\hline Allamakee & 72,111 & 54.1 & 15,792 & 12,634 & 65,696 & 0.193 \\
\hline Appanoose & 5,462 & 4.1 & 1,196 & 957 & 4,976 & 0.015 \\
\hline Audubon & 168,217 & 126.2 & 36,840 & 29,472 & 153,252 & 0.451 \\
\hline Benton & 102,216 & 76.7 & 22,385 & 17,908 & 93,123 & 0.274 \\
\hline Black Hawk & 171,797 & 128.8 & 37,624 & 30,099 & 156,514 & 0.460 \\
\hline Boone & 101,679 & 76.3 & 22,268 & 17,814 & 92,634 & 0.273 \\
\hline Bremer & 88,693 & 66.5 & 19,424 & 15,539 & 80,803 & 0.238 \\
\hline Buchanan & 191,642 & 143.7 & 41,970 & 33,576 & 174,594 & 0.514 \\
\hline Buena Vista & 298,220 & 223.7 & 65,310 & 52,248 & 271,690 & 0.799 \\
\hline Butier & 178,682 & 134.0 & 39,131 & 31,305 & 162,786 & 0.479 \\
\hline Calhoun & 171,427 & 128.6 & 37,543 & 30,034 & 156,177 & 0.459 \\
\hline Carroll & 372,598 & 279.4 & 81,599 & 65,279 & 339,452 & 0.999 \\
\hline Cass & 64,720 & 48.5 & 14,174 & 11,339 & 58,963 & 0.173 \\
\hline Cedar & 151,936 & 114.0 & 33,274 & 26,619 & 138,420 & 0.407 \\
\hline Cerro Gordo & 126,766 & 95.1 & 27,762 & 22,209 & 115,489 & 0.340 \\
\hline Cherokee & 222,808 & 167.1 & 48,795 & 39,036 & 202,987 & 0.597 \\
\hline Chickasaw & 131,490 & 98.6 & 28,796 & 23,037 & 119,793 & 0.352 \\
\hline Clarke & 67,224 & 50.4 & 14,722 & 11,778 & 61,244 & 0.180 \\
\hline Clay & 108,489 & 81.4 & 23,759 & 19,007 & 98,838 & 0.291 \\
\hline Clayton & 242,580 & 181.9 & 53,125 & 42,500 & 221,000 & 0.650 \\
\hline Clinton & 101,869 & 76.4 & 22,309 & 17,847 & 92,807 & 0.273 \\
\hline Crawford & 179,383 & 134.5 & 39,285 & 31,428 & 163,425 & 0.481 \\
\hline Dallas & 56,587 & 42.4 & 12,393 & 9,914 & 51,553 & 0.152 \\
\hline Davis & 123,102 & 92.3 & 26,959 & 21,567 & 112,151 & 0.330 \\
\hline Decatur & 39,037 & 29.3 & 8,549 & 6,839 & 35,564 & 0.105 \\
\hline Delaware & 401,729 & 301.3 & 87,979 & 70,383 & 365,991 & 1.077 \\
\hline Des Moines & 125,030 & 93.8 & 27,382 & 21,905 & 113,907 & 0.335 \\
\hline Dickinson & 45,333 & 34.0 & 9,928 & 7,942 & 41,300 & 0.121 \\
\hline Dubuque & 258,568 & 193.9 & 56,626 & 45,301 & 235,566 & 0.693 \\
\hline Emmet & 120,605 & 90.5 & 26,412 & 21,130 & 109,876 & 0.323 \\
\hline Fayette & 242,628 & 182.0 & 53,136 & 42,508 & 221,044 & 0.650 \\
\hline Floyd & 106,157 & 79.6 & 23,248 & 18,599 & 96,713 & 0.285 \\
\hline Franklin & 198,056 & 148.5 & 43,374 & 34,699 & 180,437 & 0.531 \\
\hline Fremont & 24,261 & 18.2 & 5,313 & 4,251 & 22,103 & 0.065 \\
\hline Greene & 154,717 & 116.0 & 33,883 & 27,106 & 140,953 & 0.415 \\
\hline Grundy & 156,834 & 117.6 & 34,347 & 27,477 & 142,882 & 0.420 \\
\hline Guthrie & 81,536 & 61.2 & 17,856 & 14,285 & 74,283 & 0.219 \\
\hline Hamilton & 448,312 & 336.2 & 98,180 & 78,544 & 408,430 & 1.201 \\
\hline Hancock & 174,621 & 131.0 & 38,242 & 30,594 & 159,087 & 0.468 \\
\hline Hardin & 395,359 & 296.5 & 86,584 & 69,267 & 360,188 & 1.060 \\
\hline Harrison & 66,383 & 49.8 & 14,538 & 11,630 & 60,478 & 0.178 \\
\hline Henry & 88,271 & 66.2 & 19,331 & 15,465 & 80,418 & 0.237 \\
\hline Howard & 98,979 & 74.2 & 21,676 & 17,341 & 90,174 & 0.265 \\
\hline Humboldt & 85,721 & 64.3 & 18,773 & 15,018 & $.78,095$ & 0.230 \\
\hline Ida & 120,684 & 90.5 & 26,430 & 21,144 & 109,948 & 0.323 \\
\hline
\end{tabular}




\begin{tabular}{|c|c|c|c|c|c|c|}
\hline County & $\begin{array}{c}\text { Animal } \\
\text { Numbers }\end{array}$ & $\begin{array}{c}\text { Total } \\
\text { Solids } \\
\text { (tons/day) }\end{array}$ & $\begin{array}{c}\text { Volatile } \\
\text { Solids } \\
\text { (tons/yr) }\end{array}$ & $\begin{array}{c}\text { Collectable } \\
\text { Solids } \\
\text { (tons/yr) }\end{array}$ & $\begin{array}{c}\text { Potential } \\
\text { Energy } \\
\text { (MMBtulyr) }\end{array}$ & $\begin{array}{c}\text { Potential } \\
\text { Energy } \\
\text { (MW) }\end{array}$ \\
\hline lowa & 134,113 & 100.6 & 29,371 & 23,497 & 122,182 & 0.359 \\
\hline Jackson & 89,234 & 66.9 & 19,542 & 15,634 & 81,296 & 0.239 \\
\hline Jasper & 180,743 & 135.6 & 39,583 & 31,666 & 164,664 & 0.484 \\
\hline Jefferson & 72,998 & 54.7 & 15,987 & 12,789 & 66,504 & 0.196 \\
\hline Johnson & 137,946 & 103.5 & 30,210 & 24,168 & 125,674 & 0.370 \\
\hline Jones & 147,204 & 110.4 & 32,238 & 25,790 & 134,109 & 0.395 \\
\hline Keokuk & 144,412 & 108.3 & 31,626 & 25,301 & 131,565 & 0.387 \\
\hline Kossuth & 323,029 & 242.3 & 70,743 & 56,595 & 294,292 & 0.866 \\
\hline Lee & 113,345 & 85.0 & 24,823 & 19,858 & 103,262 & 0.304 \\
\hline Linn & 80,301 & 60.2 & 17,586 & 14,069 & 73,157 & 0.215 \\
\hline Louisa & 90,189 & 67.6 & 19,751 & 15,801 & 82,166 & 0.242 \\
\hline Lucas & 19,082 & 14.3 & 4,179 & 3,343 & 17,384 & 0.051 \\
\hline Lyon & 325,619 & 244.2 & 71,311 & 57,048 & 296,652 & 0.873 \\
\hline Madison & 40,136 & 30.1 & 8,790 & 7,032 & 36,566 & 0.108 \\
\hline Mahaska & 247,819 & 185.9 & 54,272 & 43,418 & 225,773 & 0.664 \\
\hline Marion & 80,740 & 60.6 & 17,682 & 14,146 & 73,557 & 0.216 \\
\hline Marshall & 118,159 & 88.6 & 25,877 & 20,701 & 107,648 & 0.317 \\
\hline Mills & 17,544 & 13.2 & 3,842 & 3,074 & 15,983 & 0.047 \\
\hline Mitchell & 265,686 & 199.3 & 58,185 & 46,548 & 242,051 & 0.712 \\
\hline Monona & 55,490 & 41.6 & 12,152 & 9,722 & 50,554 & 0.149 \\
\hline Monroe & 45,184 & 33.9 & 9,895 & 7,916 & 41,164 & 0.121 \\
\hline Montgomery & 38,330 & 28.7 & 8,394 & 6,715 & 34,920 & 0.103 \\
\hline Muscatine & 68,777 & 51.6 & 15,062 & 12,050 & 62,659 & 0.184 \\
\hline O'Brien & 283,000 & 212.3 & 61,977 & 49,582 & 257,824 & 0.758 \\
\hline Osceola & 216,701 & 162.5 & 47,458 & 37,966 & 197,423 & 0.581 \\
\hline Page & 61,151 & 45.9 & 13,392 & 10,714 & 55,711 & 0.164 \\
\hline Palo Alto & 199,116 & 149.3 & 43,606 & 34,885 & 181,403 & 0.534 \\
\hline Plymouth & 460,965 & 345.7 & 100,951 & 80,761 & 419,958 & 1.235 \\
\hline Pocahontas & 118,209 & 88.7 & 25,888 & 20,710 & 107,693 & 0.317 \\
\hline Polk & 21,780 & 16.3 & 4,770 & 3,816 & 19,842 & 0.058 \\
\hline Pottawattamie & 87,323 & 65.5 & 19,124 & 15,299 & 79,555 & 0.234 \\
\hline Poweshiek & 109,074 & 81.8 & 23,887 & 19,110 & 99,371 & 0.292 \\
\hline Ringgold & 181,241 & 135.9 & 39,692 & 31,753 & 165,118 & 0.486 \\
\hline Sac & 350,473 & 262.9 & 76,754 & 61,403 & 319,295 & 0.939 \\
\hline Scott & 104,705 & 78.5 & 22,930 & 18,344 & 95,390 & 0.281 \\
\hline Shelby & 119,133 & 89.3 & 26,090 & 20,872 & 108,535 & 0.319 \\
\hline Sioux & 762,294 & 571.7 & 166,942 & 133,554 & 694,480 & 2.043 \\
\hline Story & 102,688 & 77.0 & 22,489 & 17,991 & 93,553 & 0.275 \\
\hline Tama & 103,275 & 77.5 & 22,617 & 18,094 & 94,088 & 0.277 \\
\hline Taylor & 41,037 & 30.8 & 8,987 & 7,190 & 37,386 & 0.110 \\
\hline Union & 56,082 & 42.1 & 12,282 & 9,826 & 51,093 & 0.150 \\
\hline Var Buren & 43,971 & 33.0 & 9,630 & 7,704 & 40,059 & 0.118 \\
\hline Wapello & 34,941 & 26.2 & 7,652 & 6,122 & 31,833 & 0.094 \\
\hline Warren & 42,339 & 31.8 & 9,272 & 7,418 & 38,573 & 0.113 \\
\hline Washington & 436,353 & 327.3 & 95,561 & 76,449 & 397,535 & 1.169 \\
\hline Wayne & 20,808 & 15.6 & 4,557 & 3,646 & 18,957 & 0.056 \\
\hline Webster & 149,935 & 112.5 & 32,836 & 26,269 & 136,597 & 0.402 \\
\hline Winnebago & 44,371 & 33.3 & 9,717 & 7,774 & 40,424 & 0.119 \\
\hline
\end{tabular}




\begin{tabular}{|c|c|c|c|c|c|c|}
\hline County & $\begin{array}{c}\text { Animal } \\
\text { Numbers }\end{array}$ & $\begin{array}{c}\text { Total } \\
\text { Solids } \\
\text { (tons/day) }\end{array}$ & $\begin{array}{l}\text { Volatile } \\
\text { Solids } \\
\text { (tonslyr) }\end{array}$ & $\begin{array}{l}\text { Collectable } \\
\text { Solids } \\
\text { (tons/yr) }\end{array}$ & $\begin{array}{c}\text { Potential } \\
\text { Energy } \\
\text { (MMBtulyr) }\end{array}$ & $\begin{array}{c}\text { Potential } \\
\text { Energy } \\
\text { (MW) }\end{array}$ \\
\hline Winneshiek & 125,534 & 94.2 & 27,492 & 21,994 & 114,366 & 0.336 \\
\hline Woodbury & 103,850 & 77.9 & 22,743 & 18,195 & 94,612 & 0.278 \\
\hline Worth & 46,325 & 34.7 & 10,145 & 8,116 & 42,204 & 0.124 \\
\hline Wright & 358,616 & 269.0 & 78,537 & 62,830 & 326,714 & 0.961 \\
\hline \multicolumn{7}{|c|}{ Poultry Layers } \\
\hline County & $\begin{array}{c}\text { Animal } \\
\text { Numbers }\end{array}$ & $\begin{array}{c}\text { Total } \\
\text { Solids } \\
\text { (tons/day) }\end{array}$ & $\begin{array}{l}\text { Volatile } \\
\text { Solids } \\
\text { (tons/yr) }\end{array}$ & $\begin{array}{l}\text { Collectable } \\
\text { Solids } \\
\text { (tons/yr) }\end{array}$ & $\begin{array}{l}\text { Potential } \\
\text { Energy } \\
\text { (MMBtu/yr) }\end{array}$ & $\begin{array}{c}\text { Potential } \\
\text { Energy } \\
\text { (MW) }\end{array}$ \\
\hline Adair & 0 & 0.0 & 0 & 0 & 0.0 & 0.000 \\
\hline Adams & 2,057 & 0.1 & 18 & 16 & 100.4 & 0.000 \\
\hline Allamakee & 413 & 0.0 & 4 & 3 & 20.2 & 0.000 \\
\hline Appanoose & 410 & 0.0 & 4 & 3 & 20.0 & 0.000 \\
\hline Audubon & 0 & 0.0 & 0 & 0 & 0.0 & 0.000 \\
\hline Benton & 0 & 0.0 & 0 & 0 & 0.0 & 0.000 \\
\hline Black Hawk & 0 & 0.0 & 0 & 0 & 0.0 & 0.000 \\
\hline Boone & 0 & 0.0 & 0 & 0 & 0.0 & 0.000 \\
\hline Bremer & 0 & 0.0 & 0 & 0 & 0.0 & 0.000 \\
\hline Buchanan & 0 & 0.0 & 0 & 0 & 0.0 & 0.000 \\
\hline Buena Vista & 0 & 0.0 & 0 & 0 & 0.0 & 0.000 \\
\hline Butler & 0 & 0.0 & 0 & 0 & 0.0 & 0.000 \\
\hline Calhoun & 340 & 0.0 & 3 & 3 & 16.6 & 0.000 \\
\hline Carroll & 600 & 0.0 & 5 & 5 & 29.3 & 0.000 \\
\hline Cass & 0 & 0.0 & 0 & 0 & 0.0 & 0.000 \\
\hline Cedar & 405 & 0.0 & 4 & 3 & 19.8 & 0.000 \\
\hline Cerro Gordo & 35,664 & 1.2 & 312 & 281 & $1,741.0$ & 0.005 \\
\hline Cherokee & 0 & 0.0 & 0 & 0 & 0.0 & 0.000 \\
\hline Chickasaw & 782 & 0.0 & 7 & 6 & 38.2 & 0.000 \\
\hline Clarke & 1,146 & 0.0 & 10 & 9 & 55.9 & 0.000 \\
\hline Clay & 0 & 0.0 & 0 & 0 & 0.0 & 0.000 \\
\hline Clayton & 449,504 & 14.6 & 3,938 & 3,544 & $21,943.8$ & 0.065 \\
\hline Clinton & 991 & 0.0 & 9 & 8 & 48.4 & 0.000 \\
\hline Crawford & 0 & 0.0 & 0 & 0 & 0.0 & 0.000 \\
\hline Dallas & 213,237 & 6.9 & 1,868 & 1,681 & $10,409.7$ & 0.031 \\
\hline Davis & 11,442 & 0.4 & 100 & 90 & 558.6 & 0.002 \\
\hline Decatur & 375 & 0.0 & 3 & 3 & 18.3 & 0.000 \\
\hline Delaware & 0 & 0.0 & 0 & 0 & 0.0 & 0.000 \\
\hline Des Moines & 309 & 0.0 & 3 & 2 & 15.1 & 0.000 \\
\hline Dickinson & 84 & 0.0 & 1 & 1 & 4.1 & 0.000 \\
\hline Dubuque & 1,337 & 0.0 & 12 & 11 & 65.3 & 0.000 \\
\hline Emmet & 0 & 0.0 & 0 & 0 & 0.0 & 0.000 \\
\hline Fayette & 0 & 0.0 & 0 & 0 & 0.0 & 0.000 \\
\hline Floyd & 0 & 0.0 & 0 & 0 & 0.0 & 0.000 \\
\hline Franklin & 0 & 0.0 & 0 & 0 & 0.0 & 0.000 \\
\hline Fremont & 123 & 0.0 & 1 & 1 & 6.0 & 0.000 \\
\hline Greene & 908 & 0.0 & 8 & 7 & 44.3 & 0.000 \\
\hline Grundy & 163,891 & 5.3 & 1,436 & 1,292 & $8,000.8$ & 0.024 \\
\hline
\end{tabular}




\begin{tabular}{|c|c|c|c|c|c|c|}
\hline County & $\begin{array}{c}\text { Animal } \\
\text { Numbers }\end{array}$ & $\begin{array}{c}\text { Total } \\
\text { Solids } \\
\text { (tons/day) }\end{array}$ & $\begin{array}{c}\text { Volatile } \\
\text { Solids } \\
\text { (tons/yr) }\end{array}$ & $\begin{array}{c}\text { Collectable } \\
\text { Solids } \\
\text { (tons/yr) }\end{array}$ & $\begin{array}{c}\text { Potential } \\
\text { Energy } \\
\text { (MMBtu/yr) }\end{array}$ & $\begin{array}{l}\text { Potential } \\
\text { Energy } \\
\text { (MW) }\end{array}$ \\
\hline Guthrie & 0 & 0.0 & 0 & 0 & 0.0 & 0.000 \\
\hline Hamilton & 12,356 & 0.4 & 108 & 97 & 603.2 & 0.002 \\
\hline Hancock & 0 & 0.0 & 0 & 0 & 0.0 & 0.000 \\
\hline Hardin & 0 & 0.0 & 0 & 0 & 0.0 & 0.000 \\
\hline Harrison & 596 & 0.0 & 5 & 5 & 29.1 & 0.000 \\
\hline Henry & 312 & 0.0 & 3 & 2 & 15.2 & 0.000 \\
\hline Howard & 1,382 & 0.0 & 12 & 11 & 67.5 & 0.000 \\
\hline Humboldt & 133,062 & 4.3 & 1,166 & 1,049 & $6,495.8$ & 0.019 \\
\hline Ida & 1,201 & 0.0 & 11 & 9 & 58.6 & 0.000 \\
\hline lowa & 813 & 0.0 & 7 & 6 & 39.7 & 0.000 \\
\hline Jackson & 0 & 0.0 & 0 & 0 & 0.0 & 0.000 \\
\hline Jasper & 0 & 0.0 & 0 & 0 & 0.0 & 0.000 \\
\hline Jefferson & 780 & 0.0 & 7 & 6 & 38.1 & 0.000 \\
\hline Johnson & 64,027 & 2.1 & 561 & 505 & $3,125.7$ & 0.009 \\
\hline Jones & 364 & 0.0 & 3 & 3 & 17.8 & 0.000 \\
\hline Keokuk & 0 & 0.0 & 0 & 0 & 0.0 & 0.000 \\
\hline Kossuth & 9,515 & 0.3 & 83 & 75 & 464.5 & 0.001 \\
\hline Lee & 1,428 & 0.0 & 13 & 11 & 69.7 & 0.000 \\
\hline Linn & 1,363 & 0.0 & 12 & 11 & 66.5 & 0.000 \\
\hline Louisa & 94 & 0.0 & 1 & 1 & 4.6 & 0.000 \\
\hline Lucas & 432 & 0.0 & 4 & 3 & 21.1 & 0.000 \\
\hline Lyon & 0 & 0.0 & 0 & 0 & 0.0 & 0.000 \\
\hline Madison & 0 & 0.0 & 0 & 0 & 0.0 & 0.000 \\
\hline Mahaska & 0 & 0.0 & 0 & 0 & 0.0 & 0.000 \\
\hline Marion & 769 & 0.0 & 7 & 6 & 37.5 & 0.000 \\
\hline Marshall & 0 & 0.0 & 0 & 0 & 0.0 & 0.000 \\
\hline Mills & 260 & 0.0 & 2 & 2 & 12.7 & 0.000 \\
\hline Mitchell & $279,987^{\circ}$ & 9.1 & 2,453 & 2,207 & $13,668.3$ & 0.040 \\
\hline Monona & 193 & 0.0 & 2 & 2 & 9.4 & 0.000 \\
\hline Monroe & 153 & 0.0 & 1 & 1 & 7.5 & 0.000 \\
\hline Montgomery & 0 & 0.0 & 0 & 0 & 0.0 & 0.000 \\
\hline Muscatine & 1,886 & 0.1 & 17 & 15 & 92.1 & 0.000 \\
\hline O'Brien & 304,897 & 9.9 & 2,671 & 2,404 & $14,884.4$ & 0.044 \\
\hline Osceola & 0 & 0.0 & 0 & 0 & 0.0 & 0.000 \\
\hline Page & 841 & 0.0 & 7 & 7 & 41.1 & 0.000 \\
\hline Palo Alto & 817,505 & 26.6 & 7,161 & 6,445 & $39,908.7$ & 0.117 \\
\hline Plymouth & 7,763 & 0.3 & 68 & 61 & 379.0 & 0.001 \\
\hline Pocahontas & 0 & 0.0 & 0 & 0 & 0.0 & 0.000 \\
\hline Polk & 263 & 0.0 & 2 & 2 & 12.8 & 0.000 \\
\hline Pottawattamie & 907 & 0.0 & 8 & 7 & 44.3 & 0.000 \\
\hline Poweshiek & 0 & 0.0 & 0 & 0 & 0.0 & 0.000 \\
\hline Ringgold & 303 & 0.0 & 3 & 2 & 14.8 & 0.000 \\
\hline Sac & 96,168 & 3.1 & 842 & 758 & $4,694.7$ & 0.014 \\
\hline Scott & 2,064 & 0.1 & 18 & 16 & 100.8 & 0.000 \\
\hline Shelby & 582 & 0.0 & 5 & 5 & 28.4 & 0.000 \\
\hline Sioux & $1,573,881$ & 51.2 & 13,787 & 12,408 & $76,833.3$ & 0.226 \\
\hline Story & 1,132 & 0.0 & 10 & 9 & 55.3 & 0.000 \\
\hline Tama & 1,107 & 0.0 & 10 & 9 & 54.0 & 0.000 \\
\hline
\end{tabular}




\begin{tabular}{|c|c|c|c|c|c|c|}
\hline County & $\begin{array}{c}\text { Animal } \\
\text { Numbers }\end{array}$ & $\begin{array}{c}\text { Total } \\
\text { Solids } \\
\text { (tons/day) }\end{array}$ & $\begin{array}{l}\text { Volatile } \\
\text { Solids } \\
\text { (tons/yr) }\end{array}$ & $\begin{array}{c}\text { Collectable } \\
\text { Solids } \\
\text { (tons/yr) }\end{array}$ & $\begin{array}{l}\text { Potential } \\
\text { Energy } \\
\text { (MMBtulyr) }\end{array}$ & $\begin{array}{l}\text { Potential } \\
\text { Energy } \\
\text { (MW) }\end{array}$ \\
\hline Taylor & 0 & 0.0 & 0 & 0 & 0.0 & 0.000 \\
\hline Union & 148 & 0.0 & 1 & 1 & 7.2 & 0.000 \\
\hline Van Buren & 975 & 0.0 & 9 & 8 & 47.6 & 0.000 \\
\hline Wapello. & 526 & 0.0 & 5 & 4 & 25.7 & 0.000 \\
\hline Warren & 620 & 0.0 & 5 & 5 & 30.3 & 0.000 \\
\hline Washington & 399,737 & 13.0 & 3,502 & 3,152 & $19,514.3$ & 0.057 \\
\hline Wayne & 230 & 0.0 & 2 & 2 & 11.2 & 0.000 \\
\hline Webster & 0 & 0.0 & 0 & 0 & 0.0 & 0.000 \\
\hline Winnebago & 619 & 0.0 & 5 & 5 & 30.2 & 0.000 \\
\hline Winneshiek & 0 & 0.0 & 0 & 0 & 0.0 & 0.000 \\
\hline Woodbury & 1,267 & 0.0 & 11 & 10 & 61.9 & 0.000 \\
\hline Worth & 0 & 0.0 & 0 & 0 & 0.0 & 0.000 \\
\hline Wright & 0 & 0.0 & 0 & 0 & 0.0 & 0.000 \\
\hline \multicolumn{7}{|c|}{ Poultry Boilers } \\
\hline County & $\begin{array}{c}\text { Animal } \\
\text { Numbers }\end{array}$ & $\begin{array}{c}\text { Total } \\
\text { Solids } \\
\text { (tons/day) }\end{array}$ & $\begin{array}{c}\text { Volatile } \\
\text { Solids } \\
\text { (tons/yr) }\end{array}$ & $\begin{array}{l}\text { Collectable } \\
\text { Solids } \\
\text { (tons/yr) }\end{array}$ & $\begin{array}{c}\text { Potential } \\
\text { Energy } \\
\text { (MMBtulyr) }\end{array}$ & $\begin{array}{c}\text { Potential } \\
\text { Energy } \\
\text { (MW) }\end{array}$ \\
\hline Adair & 285 & 0.0 & 2 & 1 & 9.3 & 0.000 \\
\hline Adams & 0 & 0.0 & 0 & 0 & 0.0 & 0.000 \\
\hline Allamakee & 0 & 0.0 & 0 & 0 & 0.0 & 0.000 \\
\hline Appanoose & 533 & 0.0 & 3 & 3 & 17.3 & 0.000 \\
\hline Audubon & 124 & 0.0 & 1 & 1 & 4.0 & 0.000 \\
\hline Benton & 586 & 0.0 & 3 & 3 & 19.1 & 0.000 \\
\hline Black Hawk & 218 & 0.0 & 1 & 1 & 7.1 & 0.000 \\
\hline Boone & 508 & 0.0 & 3 & 3 & 16.5 & 0.000 \\
\hline Bremer & 1,435 & 0.0 & 8 & 8 & 46.7 & 0.000 \\
\hline Buchanan & 39,013 & 0.8 & 228 & 205 & $1,269.7$ & 0.004 \\
\hline Buena Vista & 853 & 0.0 & 5 & 4 & 27.8 & 0.000 \\
\hline Butler & 0 & 0.0 & 0 & 0 & 0.0 & 0.000 \\
\hline Calhoun & 3,048 & 0.1 & 18 & 16 & 99.2 & 0.000 \\
\hline Carroll & 266 & 0.0 & 2 & 1 & 8.7 & 0.000 \\
\hline Cass & 0 & 0.0 & 0 & 0 & 0.0 & 0.000 \\
\hline Cedar & 426 & 0.0 & 2 & 2 & 13.9 & 0.000 \\
\hline Cerro Gordo & 40 & 0.0 & 0 & 0 & 1.3 & 0.000 \\
\hline Cherokee & 0 & 0.0 & 0 & 0 & 0.0 & 0.000 \\
\hline Chickasaw & 420 & 0.0 & 2 & 2 & 13.7 & 0.000 \\
\hline Clarke & 0 & 0.0 & 0 & 0 & 0.0 & 0.000 \\
\hline Clay & 381 & 0.0 & 2 & 2 & 12.4 & 0.000 \\
\hline Clayton & 219 & 0.0 & 1 & 1 & 7.1 & 0.000 \\
\hline Clinton & 570 & 0.0 & 3 & 3 & 18.6 & 0.000 \\
\hline Crawford & 765 & 0.0 & 4 & 4. & 24.9 & 0.000 \\
\hline Dallas & 260 & 0.0 & 2 & 1 & 8.5 & 0.000 \\
\hline Davis & 413 & 0.0 & 2 & 2 & 13.4 & 0.000 \\
\hline Decatur & 809 & 0.0 & 5 & 4 & 26.3 & 0.000 \\
\hline Delaware & 258 & 0.0 & 2 & 1 & 8.4 & 0.000 \\
\hline Des Moines & 245 & 0.0 & 1 & 1 & 8.0 & 0.000 \\
\hline
\end{tabular}




\begin{tabular}{|c|c|c|c|c|c|c|}
\hline County & $\begin{array}{c}\text { Animal } \\
\text { Numbers }\end{array}$ & $\begin{array}{c}\text { Total } \\
\text { Solids } \\
\text { (tons/day) }\end{array}$ & $\begin{array}{l}\text { Volatile } \\
\text { Solids } \\
\text { (tons/yr) }\end{array}$ & $\begin{array}{c}\text { Collectable } \\
\text { Solids } \\
\text { (tons/yr) }\end{array}$ & $\begin{array}{c}\text { Potential } \\
\text { Energy } \\
\text { (MMBtu/yr) }\end{array}$ & $\begin{array}{c}\text { Potential } \\
\text { Energy } \\
\text { (MW) }\end{array}$ \\
\hline Dickinson & 0 & 0.0 & 0 & 0 & 0.0 & 0.000 \\
\hline Dubuque & 713 & 0.0 & 4 & 4 & 23.2 & 0.000 \\
\hline Emmet & 0 & 0.0 & 0 & 0 & 0.0 & 0.000 \\
\hline Fayette & 339 & 0.0 & 2 & 2 & 11.0 & 0.000 \\
\hline Floyd & 485 & 0.0 & 3 & 3 & 15.8 & 0.000 \\
\hline Franklin & 770 & 0.0 & 4 & 4 & 25.1 & 0.000 \\
\hline Fremont & 0 & 0.0 & 0 & 0 & 0.0 & 0.000 \\
\hline Greene & 2,709 & 0.1 & 16 & 14 & 88.2 & 0.000 \\
\hline Grundy & 1,500 & 0.0 & 9 & 8 & 48.8 & 0.000 \\
\hline Guthrie & 602 & 0.0 & 4 & 3 & 19.6 & 0.000 \\
\hline Hamilton & 229 & 0.0 & 1 & 1 & 7.5 & 0.000 \\
\hline Hancock & 460 & 0.0 & 3 & 2 & 15.0 & 0.000 \\
\hline Hardin & 315 & 0.0 & 2 & 2 & 10.3 & 0.000 \\
\hline Harrison & 435 & 0.0 & 3 & 2 & 14.2 & 0.000 \\
\hline Henry & 350 & 0.0 & 2 & 2 & 11.4 & 0.000 \\
\hline Howard & 764 & 0.0 & 4 & 4 & 24.9 & 0.000 \\
\hline Humboldt & 0 & 0.0 & 0 & 0 & 0.0 & 0.000 \\
\hline Ida & 1,495 & 0.0 & 9 & 8 & 48.7 & 0.000 \\
\hline lowa & 1,254 & 0.0 & 7 & 7 & 40.8 & 0.000 \\
\hline Jackson & 597 & 0.0 & 3 & 3 & 19.4 & 0.000 \\
\hline Jasper & 234 & 0.0 & 1 & 1 & 7.6 & 0.000 \\
\hline Jefferson & 162 & 0.0 & 1 & 1 & 5.3 & 0.000 \\
\hline Johnson & 4,023 & 0.1 & 23 & 21 & 130.9 & 0.000 \\
\hline Jones & 338 & 0.0 & 2 & 2 & 11.0 & 0.000 \\
\hline Keokuk & 900 & 0.0 & 5 & 5 & 29.3 & 0.000 \\
\hline Kossuth & 2,135 & 0.0 & 12 & 11 & 69.5 & 0.000 \\
\hline Lee & 72 & 0.0 & 0 & 0 & 2.3 & 0.000 \\
\hline Linn & 328 & 0.0 & 2 & 2 & 10.7 & 0.000 \\
\hline Louisa & 0 & 0.0 & 0 & 0 & 0.0 & 0.000 \\
\hline Lucas & 1,020 & 0.0 & 6 & 5 & 33.2 & 0.000 \\
\hline Lyon & 0 & 0.0 & 0 & 0 & 0.0 & 0.000 \\
\hline Madison & 606 & 0.0 & 4 & 3 & 19.7 & 0.000 \\
\hline Mahaska & 0 & 0.0 & 0 & 0 & 0.0 & 0.000 \\
\hline Marion & 0 & 0.0 & 0 & 0 & 0.0 & 0.000 \\
\hline Marshall & 400 & 0.0 & 2 & 2 & 13.0 & 0.000 \\
\hline Mills & 175 & 0.0 & 1 & 1 & 5.7 . & 0.000 \\
\hline Mitchell & 903 & 0.0 & 5 & 5 & 29.4 & 0.000 \\
\hline Monona & 450 & 0.0 & 3 & 2 & 14.6 & 0.000 \\
\hline Monroe & 0 & 0.0 & 0 & 0 & 0.0 & 0.000 \\
\hline Montgomery & 200 & 0.0 & 1 & 1 & 6.5 & 0.000 \\
\hline Muscatine & 490 & 0.0 & 3 & 3 & 15.9 & 0.000 \\
\hline O'Brien & 175 & 0.0 & 1 & 1 & 5.7 & 0.000 \\
\hline Osceola & 0 & 0.0 & 0 & 0 & 0.0 & 0.000 \\
\hline Page & 0 & 0.0 & 0 & 0 & 0.0 & 0.000 \\
\hline Palo Alto & 1,220 & 0.0 & 7 & 6 & 39.7 & 0.000 \\
\hline Plymouth & 199 & 0.0 & 1 & 1 & 6.5 & 0.000 \\
\hline Pocahontas & 0 & 0.0 & 0 & 0 & 0.0 & 0.000 \\
\hline Polk & 615 & 0.0 & 4 & 3 & 20.0 & 0.000 \\
\hline
\end{tabular}




\begin{tabular}{|c|c|c|c|c|c|c|}
\hline County & $\begin{array}{c}\text { Animal } \\
\text { Numbers }\end{array}$ & $\begin{array}{c}\text { Total } \\
\text { Solids } \\
\text { (tons/day) }\end{array}$ & $\begin{array}{c}\text { Volatile } \\
\text { Solids } \\
\text { (tons/yr) }\end{array}$ & $\begin{array}{c}\text { Collectable } \\
\text { Solids } \\
\text { (tons/yr) }\end{array}$ & $\begin{array}{l}\text { Potential } \\
\text { Energy } \\
\text { (MMBtu/yr) }\end{array}$ & $\begin{array}{c}\text { Potential } \\
\text { Energy } \\
\text { (MW) }\end{array}$ \\
\hline Pottawattamie & 1,184 & 0.0 & 7 & 6 & 38.5 & 0.000 \\
\hline Poweshiek & 252 & 0.0 & 1 & 1 & 8.2 & 0.000 \\
\hline Ringgold & 0 & 0.0 & 0 & 0 & 0.0 & 0.000 \\
\hline Sac & 517 & 0.0 & 3 & 3 & 16.8 & 0.000 \\
\hline Scott & 0 & 0.0 & 0 & 0 & 0.0 & 0.000 \\
\hline Shelby & 679 & 0.0 & 4 & 4 & 22.1 & 0.000 \\
\hline Sioux & 221,062 & 4.8 & 1,291 & 1,162 & 7.194 .5 & 0.021 \\
\hline Story & 894 & 0.0 & 5 & 5 & 29.1 & 0.000 \\
\hline Tama & 925 & 0.0 & 5 & 5 & 30.1 & 0.000 \\
\hline Taylor & 0 & 0.0 & 0 & 0 & 0.0 & 0.000 \\
\hline Union & 0 & 0.0 & 0 & 0 & 0.0 & 0.000 \\
\hline Van Buren & 312 & 0.0 & 2 & 2 & 10.2 & 0.000 \\
\hline Wapello & 0 & 0.0 & 0 & 0 & 0.0 & 0.000 \\
\hline Warren & 525 & 0.0 & 3 & 3 & 17.1 & 0.000 \\
\hline Washington & 0 & 0.0 & 0 & 0 & 0.0 & 0.000 \\
\hline Wayne & 0 & 0.0 & 0 & 0 & 0.0 & 0.000 \\
\hline Webster & 0 & 0.0 & 0 & 0 & 0.0 & 0.000 \\
\hline Winnebago & 122 & 0.0 & 1 & 1 & 4.0 & 0.000 \\
\hline Winneshiek & 688 & 0.0 & 4 & 4 & 22.4 & 0.000 \\
\hline Woodbury & 439 & 0.0 & 3 & 2 & 14.3 & 0.000 \\
\hline Worth & 324 & 0.0 & 2 & 2 & 10.5 & 0.000 \\
\hline Wright & 276 & 0.0 & 2 & 1 & 9.0 & 0.000 \\
\hline \multicolumn{7}{|c|}{ Poultry-Turkeys } \\
\hline County & $\begin{array}{c}\text { Animal } \\
\text { Numbers }\end{array}$ & $\begin{array}{c}\text { Total } \\
\text { Solids } \\
\text { (tons/day) }\end{array}$ & $\begin{array}{l}\text { Volatile } \\
\text { Solids } \\
\text { (tons/yr) }\end{array}$ & $\begin{array}{c}\text { Collectable } \\
\text { Solids } \\
\text { (tons/yr) }\end{array}$ & $\begin{array}{l}\text { Potential } \\
\text { Energy } \\
\text { (MMBtu/yr) }\end{array}$ & $\begin{array}{l}\text { Potential } \\
\text { Energy } \\
\text { (MW) }\end{array}$ \\
\hline Adair & 0 & 0.0 & 0 & 0 & 0.0 & 0.000 \\
\hline Adams & 0 & 0.0 & 0 & 0 & 0.0 & 0.000 \\
\hline Allamakee & 0 & 0.0 & 0 & 0 & 0.0 & 0.000 \\
\hline Appanoose & 0 & 0.0 & 0 & 0 & 0.0 & 0.000 \\
\hline Audubon & 0 & 0.0 & 0 & 0 & 0.0 & 0.000 \\
\hline Benton & 33 & 0.0 & 1. & 0 & 2.9 & 0.000 \\
\hline Black Hawk & 0 & 0.0 & 0 & 0 & 0.0 & 0.000 \\
\hline Boone & 0 & 0.0 & 0 & 0 & 0.0 & 0.000 \\
\hline Bremer & 0 & 0.0 & 0 & 0 & 0.0 & 0.000 \\
\hline Buchanan & 0 & 0.0 & 0 & 0 & 0.0 & 0.000 \\
\hline Buena Vista & 652,179 & 55.4 & 15,473 & 9,284 & $57,485.1$ & 0.169 \\
\hline Butler & 0 & 0.0 & 0 & 0 & 0.0 & 0.000 \\
\hline Calhoun & 0 & 0.0 & 0 & 0 & 0.0 & 0.000 \\
\hline Carroll & 10 & 0.0 & 0 & 0 & 0.9 & 0.000 \\
\hline Cass & 0 & 0.0 & 0 & 0 & 0.0 & 0.000 \\
\hline Cedar & 0 & 0.0 & 0 & 0 & 0.0 & 0.000 \\
\hline Cerro Gordo & 8 & 0.0 & 0 & 0 & 0.7 & 0.000 \\
\hline Cherokee & 0 & 0.0 & 0 & 0 & 0.0 & 0.000 \\
\hline Chickasaw & 0 & 0.0 & 0 & 0 & 0.0 & 0.000 \\
\hline Clarke & 0 & 0.0 & 0 & 0 & 0.0 & 0.000 \\
\hline
\end{tabular}




\begin{tabular}{|c|c|c|c|c|c|c|}
\hline County & $\begin{array}{c}\text { Animal } \\
\text { Numbers }\end{array}$ & $\begin{array}{c}\text { Total } \\
\text { Solids } \\
\text { (tons/day) }\end{array}$ & $\begin{array}{c}\text { Volatile } \\
\text { Solids } \\
\text { (tons/yr) }\end{array}$ & $\begin{array}{l}\text { Collectable } \\
\text { Solids } \\
\text { (tonslyr) }\end{array}$ & $\begin{array}{l}\text { Potential } \\
\text { Energy } \\
\text { (MMBtu/yr) }\end{array}$ & $\begin{array}{c}\text { Potential } \\
\text { Energy } \\
\text { (MW) }\end{array}$ \\
\hline Clay & 0 & 0.0 & 0 & 0 & 0.0 & 0.000 \\
\hline Clayton & 0 & 0.0 & 0 & 0 & 0.0 & 0.000 \\
\hline Clinton & 161 & 0.0 & 4 & 2 & 14.2 & 0.000 \\
\hline Crawford & 25 & 0.0 & 1 & 0 & 2.2 & 0.000 \\
\hline Dallas & 0 & 0.0 & 0 & 0 & 0.0 & 0.000 \\
\hline Davis & 18 & 0.0 & 0 & 0 & 1.6 & 0.000 \\
\hline Decatur & 24 & 0.0 & 1 & 0 & 2.1 & 0.000 \\
\hline Delaware & 22 & 0.0 & 1 & 0 & 1.9 & 0.000 \\
\hline Des Moines & 0 & 0.0 & 0 & 0 & 0.0 & 0.000 \\
\hline Dickinson & 0 & 0.0 & 0 & 0 & 0.0 & 0.000 \\
\hline Dubuque & 30 & 0.0 & 1 & 0 & 2.6 & 0.000 \\
\hline Emmet & 0 & 0.0 & 0 & 0 & 0.0 & 0.000 \\
\hline Fayette & 0 & 0.0 & 0 & 0 & 0.0 & 0.000 \\
\hline Floyd & 0 & 0.0 & 0 & 0 & 0.0 & 0.000 \\
\hline Franklin & 33 & 0.0 & 1 & 0 & 2.9 & 0.000 \\
\hline Fremont & 0 & 0.0 & 0 & 0 & 0.0 & 0.000 \\
\hline Greene & 19 & 0.0 & 0 & 0 & 1.7 & 0.000 \\
\hline Grundy & 0 & 0.0 & 0 & 0 & 0.0 & 0.000 \\
\hline Guthrie & 0 & 0.0 & 0 & 0 & 0.0 & 0.000 \\
\hline Hamilton & 655,000 & 55.7 & 15,540 & 9,324 & $57,733.7$ & 0.170 \\
\hline Hancock & 55 & 0.0 & 1 & 1 & 4.8 & 0.000 \\
\hline Hardin & 0 & 0.0 & 0 & 0 & 0.0 & 0.000 \\
\hline Harrison & 0 & 0.0 & 0 & 0 & 0.0 & 0.000 \\
\hline Henry & 128,362 & 10.9 & 3,045 & 1,827 & $11,314.2$ & 0.033 \\
\hline Howard & 9 & 0.0 & 0 & 0 & 0.8 & 0.000 \\
\hline Humboldt & 0 & 0.0 & 0 & 0 & 0.0 & 0.000 \\
\hline Ida & 0 & 0.0 & 0 & 0 & 0.0 & 0.000 \\
\hline lowa & 0 & 0.0 & 0 & 0 & 0.0 & 0.000 \\
\hline Jackson & 177 & 0.0 & 4 & 3 & 15.6 & 0.000 \\
\hline Jasper & 0 & 0.0 & 0 & 0 & 0.0 & 0.000 \\
\hline Jefferson & 0 & 0.0 & 0 & 0 & 0.0 & 0.000 \\
\hline Johnson & 35,214 & 3.0 & 835 & 501 & $3,103.9$ & 0.009 \\
\hline Jones & 32 & 0.0 & 1 & 0 & 2.8 & 0.000 \\
\hline Keokuk & 0 & 0.0 & 0 & 0 & 0.0 & $0: 000$ \\
\hline Kossuth & 75 & 0.0 & 2 & 1 & 6.6 & 0.000 \\
\hline Lee & 0 & 0.0 & 0 & 0 & 0.0 & 0.000 \\
\hline Linn & 116 & 0.0 & 3 & 2 & 10.2 & 0.000 \\
\hline Louisa & 0 & 0.0 & 0 & 0 & 0.0 & 0.000 \\
\hline Lucas & 0 & 0.0 & 0 & 0 & 0.0 & 0.000 \\
\hline Lyon & 35 & 0.0 & 1 & 0 & 3.1 & 0.000 \\
\hline Madison & 72 & 0.0 & 2 & 1 & 6.3 & 0.000 \\
\hline Mahaska & 0 & 0.0 & .0 & 0 & 0.0 & 0.000 \\
\hline Marion & 0 & 0.0 & 0 & 0 & 0.0 & 0.000 \\
\hline Marshall & 0 & 0.0 & 0 & 0 & 0.0 & 0.000 \\
\hline Mills & 0 & 0.0 & 0 & 0 & 0.0 & 0.000 \\
\hline Mitchell & 0 & 0.0 & 0 & 0 & 0.0 & 0.000 \\
\hline Monona & 41 & 0.0 & 1 & 1 & 3.6 & 0.000 \\
\hline Monroe & 0 & 0.0 & 0 & 0 & 0.0 & 0.000 \\
\hline
\end{tabular}




\begin{tabular}{|c|c|c|c|c|c|c|}
\hline County & $\begin{array}{c}\text { Animal } \\
\text { Numbers }\end{array}$ & $\begin{array}{c}\text { Total } \\
\text { Solids } \\
\text { (tons/day) }\end{array}$ & $\begin{array}{l}\text { Volatile } \\
\text { Solids } \\
\text { (tons/yr) }\end{array}$ & $\begin{array}{c}\text { Collectable } \\
\text { Solids } \\
\text { (tons/yr) }\end{array}$ & $\begin{array}{c}\text { Potential } \\
\text { Energy } \\
\text { (MMBtu/yr) }\end{array}$ & $\begin{array}{c}\text { Potential } \\
\text { Energy } \\
\text { (MW) }\end{array}$ \\
\hline Montgomery & 0 & 0.0 & 0 & 0 & 0.0 & 0.000 \\
\hline Muscatine & 0 & 0.0 & 0 & 0 & 0.0 & 0.000 \\
\hline O'Brien & 0 & 0.0 & 0 & 0 & 0.0 & 0.000 \\
\hline Osceola & 0 & 0.0 & 0 & 0 & 0.0 & 0.000 \\
\hline Page & 0 & 0.0 & 0 & 0 & 0.0 & 0.000 \\
\hline Palo Alto & 0 & 0.0 & 0 & 0 & 0.0 & 0.000 \\
\hline Plymouth & 41 & 0.0 & 1 & 1 & 3.6 & 0.000 \\
\hline Pocahontas & 0 & 0.0 & 0 & 0 & 0.0 & 0.000 \\
\hline Polk & 47 & 0.0 & 1 & 1 & 4.1 & 0.000 \\
\hline Pottawattamie & 31 & 0.0 & 1 & 0 & 2.7 & 0.000 \\
\hline Poweshiek & 6 & 0.0 & 0 & 0 & 0.5 & 0.000 \\
\hline Ringgold & 7 & 0.0 & 0 & 0 & 0.6 & 0.000 \\
\hline Sac & 0 & 0.0 & 0 & 0 & 0.0 & 0.000 \\
\hline Scott & 0 & 0.0 & 0 & 0 & 0.0 & 0.000 \\
\hline Shelby & 0 & 0.0 & 0 & 0 & 0.0 & 0.000 \\
\hline Sioux & 6 & 0.0 & 0 & 0 & 0.5 & 0.000 \\
\hline Story & 0 & 0.0 & 0 & 0 & 0.0 & 0.000 \\
\hline Tama & 34 & 0.0 & 1 & 0 & 3.0 & 0.000 \\
\hline Taylor & 0 & 0.0 & 0 & 0 & 0.0 & 0.000 \\
\hline Union & 117 & 0.0 & 3 & 2 & 10.3 & 0.000 \\
\hline Van Buren & 0 & 0.0 & 0 & 0 & 0.0 & 0.000 \\
\hline Wapello & 0 & 0.0 & 0 & 0 & 0.0 & 0.000 \\
\hline Warren & 45 & 0.0 & 1 & 1 & 4.0 & 0.000 \\
\hline Washington & 325,130 & 27.6 & 7,714 & 4,628 & $28,658.0$ & 0.084 \\
\hline Wayne & 10 & 0.0 & 0 & 0 & 0.9 & 0.000 \\
\hline Webster & 0 & 0.0 & 0 & 0 & 0.0 & 0.000 \\
\hline Winnebago & 0 & 0.0 & 0 & 0 & 0.0 & 0.000 \\
\hline Winneshiek & 14 & 0.0 & 0 & 0 & 1.2 & 0.000 \\
\hline Woodbury & 0 & 0.0 & 0 & 0 & 0.0 & 0.000 \\
\hline Worth & 0 & 0.0 & 0 & 0 & 0.0 & 0.000 \\
\hline Wright & 0 & 0.0 & 0 & 0 & 0.0 & 0.000 \\
\hline
\end{tabular}

